\title{
The Effect of Boundary Shape Deformation on Two-Dimensional Electrical Impedance Tomography
}

\author{
by \\ Alistair James Scott Boyle, PEng, BSc, University of Calgary
}

\author{
A thesis submitted to the \\ Faculty of Graduate Studies and Research \\ in partial fulfillment of the requirements for the degree of \\ Master of Applied Science in Biomedical Engineering \\ Ottawa-Carleton Institute for Biomedical Engineering \\ Department of Systems and Computer Engineering \\ Carleton University \\ Ottawa, Ontario, Canada, K1S 5B6
}

(C) Copyright 2010, Alistair J. S. Boyle, April 2010 


\section{Library and Archives \\ Canada \\ Published Heritage \\ Branch}

395 Wellington Street

Ottawa ON K1A ON4

Canada
Bibliothèque et

Archives Canada

Direction du

Patrimoine de l'édition

395, rue Wellington

Ottawa ON K1A ON4

Canada
Your file Votre référence
ISBN: 978-0-494-68654-6
Our file Notre référence
ISBN: $978-0-494-68654-6$

\section{NOTICE:}

The author has granted a nonexclusive license allowing Library and Archives Canada to reproduce, publish, archive, preserve, conserve, communicate to the public by telecommunication or on the Internet, loan, distribute and sell theses worldwide, for commercial or noncommercial purposes, in microform, paper, electronic and/or any other formats.

The author retains copyright ownership and moral rights in this thesis. Neither the thesis nor substantial extracts from it may be printed or otherwise reproduced without the author's permission.
AVIS:

L'auteur a accordé une licence non exclusive permettant à la Bibliothèque et Archives Canada de reproduire, publier, archiver, sauvegarder, conserver, transmettre au public par télécommunication ou par l'Internet, prêter, distribuer et vendre des thèses partout dans le monde, à des fins commerciales ou autres, sur support microforme, papier, électronique et/ou autres formats.

L'auteur conserve la propriété du droit d'auteur et des droits moraux qui protège cette thèse. $\mathrm{Ni}$ la thèse ni des extraits substantiels de celle-ci ne doivent être imprimés ou autrement reproduits sans son autorisation.
In compliance with the Canadian Privacy Act some supporting forms may have been removed from this thesis.

While these forms may be included in the document page count, their removal does not represent any loss of content from the thesis.
Conformément à la loi canadienne sur la protection de la vie privée, quelques formulaires secondaires ont été enlevés de cette thèse.

Bien que ces formulaires aient inclus dans la pagination, il n'y aura aucun contenu manquant.

\section{Canadä}


The undersigned recommend to the

Faculty of Graduate Studies and Research

acceptance of the thesis

\section{The Effect of Boundary Shape Deformation on Two-Dimensional Electrical Impedance Tomography}

Submitted by Alistair James Scott Boyle, PEng, BSc, University of Calgary in partial fulfillment of the requirements for the degree of

Master of Applied Science in Biomedical Engineering

Chair, Department of Systems and Computer Engineering

Dr. H. M. Schwartz

Thesis Supervisor

Dr. Andy Adler

April 2010, Carleton University 


\section{Abstract}

Electrical Impedance Tomography (EIT) is a method of obtaining images of interior conductivity from electrode measurements on the boundary. Using EIT, this work focuses upon boundary movement in the two-dimensional reconstruction problem. Investigations were carried out using the tools of the Finite Element Method (FEM), inverse problem theory, and conformal transformations though simulation and tank tests. The limitations of boundary movement reconstruction algorithms that assume isotropic conductivities were explored. Initial testing of the boundary movement reconstruction technique with a deformable phantom showed that the detected boundary movement still had errors. Simulations showed that these errors are likely conformal and do not introduce artifacts into the image but do result in incorrect boundary approximations and subsequent deformations of the reconstructed image. A mathematical exploration of the conformal motions in EIT for two dimensions was carried out. Finally, it was found that, with conformal boundary movements, electrode models could cause various image artifacts. 


\section{Acknowledgments}

A debt of gratitude are due the many people who made this Masters such a pleasant and rewarding experience: Dr Andy Adler, my supervisor, for his friendship, guidance, inspiration and assistance; Dr William Lionheart, for his mathematical assistance and encouragement; my coworkers, for allowing me the flexibility in working hours to do both work and school; my peers, at school for their camaraderie, encouragement and the constant challenge to do one better; our dog, for keeping my feet warm on those long nights; and finally, to my family and particularly my wife for their understanding, unflinching support of this endeavour, and the many hours spent correcting my comma-itis.

"There are things known and there are things unknown, and in between are the doors of perception"

- Jim Morrison 
To my wife, without whose love, support and encouragement this work would not have been possible 


\section{Contents}

Front Matter $\quad$ iii

Abstract .......................... iii

Acknowledgments . . . . . . . . . . . . . . . . iv

Dedication ..........................

Contents .......................... vi

List of Figures . . . . . . . . . . . . . . . . xi

List of Tables . . . . . . . . . . . . . . . . xiv

List of Acronyms \& Terminology $\ldots \ldots \ldots \ldots$. . . . . . . . . .

List of Symbols . . . . . . . . . . . . . . . . . . xvii

$\begin{array}{ll}\text { I Background } & 1\end{array}$

1 Introduction 2

1.1 Medical Tomography . . . . . . . . . . . . . . . . . . . . . . . . 3

1.2 Thesis Objectives . . . . . . . . . . . . . . . . . . . . 3

1.3 Thesis Contributions . . . . . . . . . . . . . . . . . . 4

2 Electrical Impedance Tomography 5 
2.1 Tomography ....................... 6

2.2 Electrical Impedance .................... . . 7

2.3 Electrical Safety . . . . . . . . . . . . . . . . . 7

2.4 A Brief History . . . . . . . . . . . . . . . . . . . 9

2.5 Breaking it Down . . . . . . . . . . . . . . . . 12

3 The Finite Element Method $\quad 14$

3.1 Finding Voltage on a Domain ... . . . . . . . . . . 15

3.1.1 Assumptions..................... 15

3.1.2 Derivation ................... 16

3.2 Weak Form . . . . . . . . . . . . . . . . . . . . . . 19

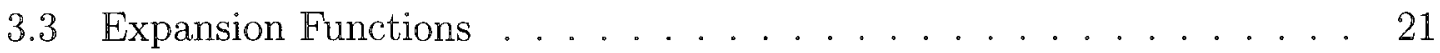

3.4 Boundary Conditions . . . . . . . . . . . . . . . . 24

4 Inverse Problems $\quad 27$

4.1 Finding Conductivity Change . . . . . . . . . . . . . 28

4.2 Regularization Methods .................. 31

4.2.1 A Bayesian View .................. 31

4.2 .2 Single Step Solutions . . . . . . . . . . . . . . . . . 33

4.2 .3 Iterative Solutions . . . . . . . . . . . . . . . . . 36

4.3 Hyper-parameter Selection . . . . . . . . . . . . . . . . 37

5 Boundary Movement $\quad 40$

5.1 An Electrode Movement Algorithm . . . . . . . . . . . . . . . . 41

5.2 Conformal Motion . . . . . . . . . . . . . . . . 48 
6 Phantom $\quad 51$

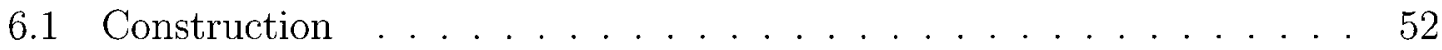

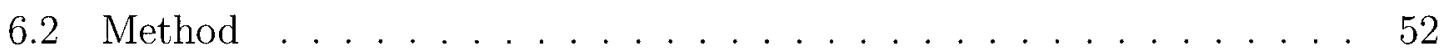

6.3 Deformations ..................... 54

6.4 Electrode Displacements . . . . . . . . . . . . . . 54

6.5 Summary and Discussion . . . . . . . . . . . . . . . 57

7 Simulated Conformal Motion $\quad 63$

7.1 Simulated Results . . . . . . . . . . . . . . . . . . 64

7.2 Summary and Discussion . . . . . . . . . . . . 67

8 Finite Element Deformations $\quad 70$

8.1 Conductivity as a Tensor . . . . . . . . . . . . . . 70

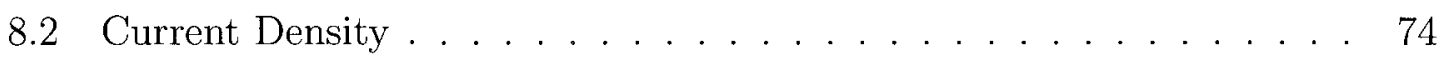

8.3 Isotropic Conductivity . . . . . . . . . . . . . . . . . . 74

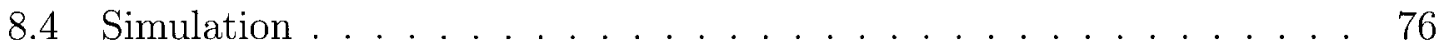

8.5 Summary and Discussion . . . . . . . . . . . . . 78

9 Conformal Motion $\quad 80$

9.1 Conformal Maps ...................... 81

9.2 Summary and Discussion . . . . . . . . . . . . . . . . . 86

10 Applied Electrode Models $\quad 88$

10.1 Contact Impedance and Electrode Area . . . . . . . . . . . . . . . . 90

10.2 Contact Impedance Variation . . . . . . . . . . . . . . . . . . . 93 
10.3 Summary and Discussion . . . . . . . . . . . . . . . 96

11 Conclusion and Future Work $\quad 100$

11.1 Recommendations . . . . . . . . . . . . . . . . . 101

11.2 Future Work . . . . . . . . . . . . . . . . . . 102

$\begin{array}{ll}\text { Appendices } & 105\end{array}$

$\begin{array}{lr}\text { A Vector Calculus } & 106\end{array}$

A.1 Determinant . . . . . . . . . . . . . 106

A.2 Vector Products . . . . . . . . . . . . . . . . . 107

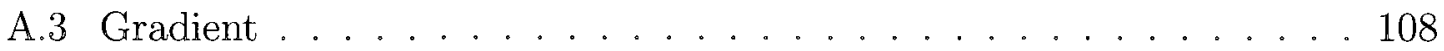

A.4 Divergence . . . . . . . . . . . . . . . . . . 108

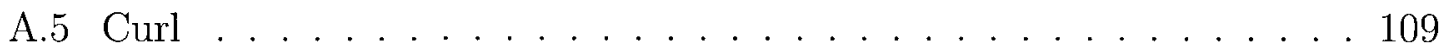

A.6 Laplacian . . . . . . . . . . . . . . . . . . . . 110

A.7 $2 \times 2$ Matrix Inverse . . . . . . . . . . . . 110

B Generalized Tikhonov Derivation $\quad 111$

B.1 Least Squares Solution . . . . . . . . . . . . . . 112

B.2 Adding Penalty Terms . . . . . . . . . . . . . . . . 112

B.3 Generalized Tikhonov Solution . . . . . . . . . . . . . . 113

B.4 Wiener Filter Form . . . . . . . . . . . . . . . . . . . . . 114 
$\begin{array}{ll}\text { References } & 116\end{array}$

Vitae

125 


\section{List of Figures}

2.1 Example EIT system, photo . . . . . . . . . . . . . . 8

2.2 Example EIT system, schematic . . . . . . . . . . . . . . 9

2.3 Physiological effects of electricity . . . . . . . . . . . . . . . . . 10

2.4 Let-go current versus frequency ................ 11

2.5 Forward and inverse problem . . . . . . . . . . . . . . . 13

2.6 Sample difference EIT simulation . . . . . . . . . . . . 13

3.12 -d $1^{\text {st }}$-order scalar interpolatory expansion functions . . . . . . . . 21

3.2 An example of an alternative expansion function . . . . . . . . . 22

4.1 Singular values of an inverse EIT problem . . . . . . . . . . . 34

4.2 Hyper-parameter selection with the L-curve . . . . . . . . . . . . . . 39

5.1 Jacobian and regularization matrices for electrode movement . . . . . 43

5.2 Joukowski Transform: a conformal mapping . . . . . . . . . . . . . . 49

5.3 A less intuitive conformal motion . . . . . . . . . . . . . . . 49

6.1 Photograph of the phantom ................... 53

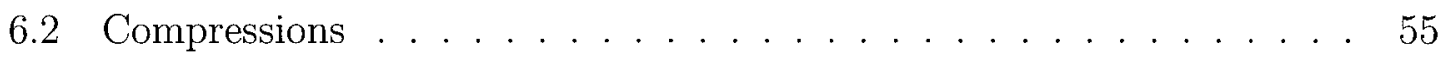


6.3 Boundary reconstruction from electrode movement: 3-point compression with conductive target . . . . . . . . . . . 57

6.4 Boundary reconstruction from electrode movement: 2-point compression with insulating target . . . . . . . . . . . . . 60 60

6.5 Boundary reconstruction from electrode movement: 2-point compression with conductive target . . . . . . . . . . . . . . . 61

6.6 Boundary reconstruction from electrode movement: 3-point compression with insulating target . . . . . . . . . . . . . . 62

7.1 Images reconstructed from three different movement patterns . . . . . 66

7.2 Simulation of two point deformation . . . . . . . . . . 68

7.3 Simulation of three point deformation . . . . . . . . . . . 69

8.1 A change of coordinate systems . . . . . . . . . . . . . . 72

8.2 Electrode current density under deformation . . . . . . . . . . 74

8.3 The two conformal FEM solutions . . . . . . . . . . . . 76

8.4 Conformal deformation convergence . . . . . . . . . . . 78

9.1 A mapping of the domain $\Omega$ to $\Omega^{\prime} \ldots \ldots$. . . . . . . . . . . . 82

9.2 A conformal mapping of a rectangular grid . . . . . . . . . . . . . 82

9.3 Derivatives of the components of a conformal vector field at the bound-

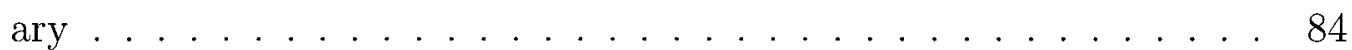

10.1 Electrode models used in mathematical proofs . . . . . . . . . . . 89

10.2 Electrode models implemented in a Finite Element Model . . . . . . . 90

10.3 Generalized electrode model . . . . . . . . . . . . . . . . . 90

10.4 Electrode area and contact impedance artifacts . . . . . . . . . . . 92 
10.5 Complex conformal deformation used in electrode model deformation simulations: $z=x+\mathrm{i} y ; z \rightarrow z+0.4 z^{2} \ldots \ldots \ldots 93$

10.6 Difference images, 2 targets $\ldots \ldots \ldots \ldots$

10.7 Difference images, electrode noise . . . . . . . . . . . . . 95

10.8 Electrode noise versus artifact amplitude $\# 1 \ldots \ldots$

10.9 Electrode noise versus artifact amplitude \#2 $\ldots \ldots \ldots$. . . . . 97

A.1 Parallelogram . . . . . . . . . . . . . . . 107 


\section{List of Tables}

6.1 Boundary Reconstruction Accuracy . . . . . . . . . . . . 58

10.1 Electrode Model Behaviour under Deformation . . . . . . . . . . . . 93 


\section{List of Acronyms \& Terminology}

AAM Artifact Amplitude Measure

APT Applied Potential Tomography

CEM Complete Electrode Model

CM Conditional Mean

CT Computed Tomography

EIDORS Electrical Impedance and Diffuse Optics Reconstruction Software

EIT Electrical Impedance Tomography

FEM Finite Element Method

GCV Generalized Cross-Validation

MCMC Markov Chain Monte Carlo

MAP Maximum A Posteriori

ML Maximum Likelihood

MRI Magnetic Resonance Imaging 
PD-IPM Primal-Dual Interior Point Method

PDE Partial Differential Equation

PEM Point Electrode Model

SEM Shunt Electrode Model

SNR Signal-to-Noise Ratio

SVD Singular Value Decomposition

TSVD Truncated SVD

TV Total Variation

in vivo within the living (latin)

in vitro within the glass (latin), in an artificial environment 


\section{List of Symbols}

a scalar

a vector $\quad\left[\begin{array}{lll}a_{1} & a_{2} & a_{3}\end{array}\right]^{\top}$

A matrix $\left[\begin{array}{lll}\mathbf{a}_{1} & \mathbf{a}_{2} & \mathbf{a}_{3}\end{array}\right]$

$\mathbf{A}^{\top} \quad$ matrix transpose

$\mathbf{A}^{*} \quad$ matrix complex conjugate transpose $\quad \mathbf{A}^{*}=\mathbf{A}^{\top}, \mathbf{A} \in \mathbb{R}$

$\mathbf{A}^{\dagger} \quad$ Moore-Penrose pseudoinverse

$\mathbf{A} \cdot \mathbf{B} \quad$ dot product

$\mathbf{A} \times \mathbf{B}$ cross product

J Jacobian

$\nabla a \quad$ gradient operator

$\nabla \cdot \mathbf{A} \quad$ divergence operator

$\nabla \times \mathbf{A} \quad$ curl operator

$\oint x d S \quad$ surface integral of $x$ on the surface $S$ 
$\frac{\partial}{\partial x} \quad$ partial derivative with respect to $x$

$\sigma \quad$ conductivity, reciprocal of resistivity $[\mathrm{S} / \mathrm{m}=1 / \Omega \cdot \mathrm{m}]$

$\omega \quad$ frequency $[\mathrm{Hz}]$

$\phi \quad$ potential $[\mathrm{V}]$

$\rho \quad$ charge density $\left[\mathrm{C} / \mathrm{m}^{3}\right]$

$\gamma \quad$ admittivity $[\mathrm{S} / \mathrm{m}](\sigma+\mathrm{i} \varepsilon$

$\varepsilon \quad$ susceptibility $[\mathrm{S} / \mathrm{m} \cdot \mathrm{Hz}]$

$\mu \quad$ mean

$\sigma \quad$ standard deviation

$\sigma^{2} \quad$ variance

$\Omega \quad$ domain, the region of interest

$\partial \Omega \quad$ boundary of the domain $\Omega$

$\hat{n} \quad$ unit vector, outward normal from the boundary $\partial \Omega$

$\Psi \quad$ expansion function

$J \quad$ current density $\left[\mathrm{A} / \mathrm{m}^{2}\right]$

B magnetic flux density $\left[\mathrm{T}=\mathrm{V} \cdot \mathrm{s} / \mathrm{m}^{2}\right]$

D electric flux density $\left[\mathrm{C} / \mathrm{m}^{2}=\mathrm{N} / \mathrm{V} \cdot \mathrm{m}^{2}\right]$

E electric field $[\mathrm{V} / \mathrm{m}=\mathrm{N} / \mathrm{C}]$ 

$\mathbf{H} \quad$ magnetic field $[\mathrm{A} / \mathrm{m}]$
$\epsilon_{0} \quad$ magnetic constant, permittivity of free space $[\mathrm{F} / \mathrm{m}]$
$\mu_{0} \quad$ electric constant, permeability of free space $\left[H / m=N / A^{2}\right]$
i imaginary number, $\sqrt{-1}$

A $\quad$ FEM system matrix

C connectivity matrix

D unconnected FEM conductivity matrix

L regularization matrix

$\mathrm{R}$ regularization covariance matrix $\sum_{x}^{-1}=\mathrm{R}=\mathrm{L}^{\top} \mathrm{L}$

S unconnected FEM shape matrix

T measurement selection matrix $\mathbf{v}=\mathbf{T x}$

W measurement covariance matrix $\sum_{n}^{-1}=\mathbf{W}$

b boundary currents

c change in conductivity $\mathrm{c}=\Delta \sigma$

r electrode movement

v boundary voltages

x voltages everywhere

$\mathbf{z} \quad$ change in boundary voltages $\mathbf{z}=\Delta \mathbf{v}$ 


\section{Part I}

\section{Background}




\section{Chapter 1}

\section{Introduction}

This thesis investigates the effect of shape deformation on two-dimensional Electrical Impedance Tomography (EIT). EIT is an electromagnetic modality that is inherently three-dimensional. $[1,2]$ This thesis focuses on two dimensions, rather than three, for the particular properties that occur with this two-dimensional approximation.

EIT is a means of obtaining an image of the internal conductivity of an object without resorting to destructive or invasive testing. In biomedical EIT, for example, sixteen electrodes and an additional ground electrode are attached around the circumference of the chest. Current is applied across pairs of electrodes, and simultaneously, voltage measurements are taken at the other electrodes. Another pair of electrodes are selected, and the process is repeated. A number of calculations are performed on these results to obtain an image of the internal conductivity distribution or the change in conductivity.[3] This thesis focuses on those calculations and how they are affected by deformations of the object. 


\subsection{Medical Tomography}

Medical tomography plays a critical role in modern Western healthcare.[4] Diagnostic tools such as Computed Tomography (CT) and Magnetic Resonance Imaging (MRI) are regularly called upon to assist in the determination of a course of treatment. They are used to investigate new pathologies and monitor the progress of treatments. [5]

These tools have become ubiquitous in hospitals because they provide a noninvasive, non-destructive method of testing hypotheses and searching for answers. They are used despite their prohibitive monetary costs [6]; the benefits they provide far outweigh those costs.

EIT offers a similar opportunity by providing a different type of information to investigators: conductivity. EIT has existed nearly as long as MRI but has not achieved widespread acceptance in the medical community. The issues with EIT from a clinical point of view are generally image quality and ability to provide predictive diagnostic information. EIT is relatively low resolution, has artifacts in the images, and can distort the image in unexpected ways rendering anatomical representations difficult. Despite this, EIT provides many benefits since it is inexpensive, portable, and non-invasive It also provides novel diagnostic information, conductivity, not available through other currently used modalities. EIT presents an interesting challenge, and the potential for significant benefit in medical diagnosis.[7]

\subsection{Thesis Objectives}

The objective of this thesis is to explore the effect that specific shape deformations have on two-dimensional EIT reconstructions through theoretical explorations and 
the use of simulation and tank studies. These investigations employ the Finite Element Method (FEM), inverse problem theory, and conformal transformations to explore the problem space. The focus is upon algorithms that assume isotropic conductivities such as those found in lung imaging.

\subsection{Thesis Contributions}

This thesis offers two novel contributions. First, an EIT reconstruction technique that adapts to boundary deformation is evaluated in vitro through the construction of a two-dimensional, deformable phantom. The algorithm is found to be incapable of discovering a specific class of deformations, the conformal motions. These conformal deformations are explored mathematically in an EIT context. A differential interpretation of these results is provided in a form more recognizable to the engineering community. Second, the effect of electrode models under these deformations is explored, and it is found that the Complete Electrode Model (CEM) can cause significant artifacts when the electrode does not deform in the same manner as the boundary, as is likely to occur in vivo. In addition, it is shown that contact impedance decreases result in significant artifacts while contact impedance increases do not.

These results have been published in the proceedings of the $g^{\text {th }}$ International Conference on Biomedical Applications of Electrical Impedance Tomography, Dartmouth, USA (EIT 2008) and the $10^{\text {th }}$ International Conference on Biomedical Applications of Electrical Impedance Tomography, Manchester, UK (EIT 2009).[8, 9] Software modifications supporting this work have been contributed to the Electrical Impedance and Diffuse Optics Reconstruction Software (EIDORS) package [10] under the GNU General Public License. 


\section{Chapter 2}

\section{Electrical Impedance Tomography}

Tomography is "a method of producing [an] ... image of the internal structures of a solid object (as the human body or the earth) by the observation and recording of the differences in the effects on the passage of waves of energy impinging on those structures." [11] It is, in short, a technique for making images, with all the art and science that involves. Science, in the sense that the images are created using measurements that are interpreted from fundamental physical and mathematical principles and manipulated using a multitude of mathematical techniques. Art, in the sense that the resulting images are ultimately intended to present information to physicians, technicians, equipment operators, or other individuals and systems in various fields to aid in decision making.

Electrical Impedance Tomography (EIT) is a form of tomography, and thus, a knowledge of what types of tomography currently exist provides context for understanding where EIT fits in the tomography spectrum. 


\subsection{Tomography}

There are a wide variety of tomography methods, and they vary with the underlying physical phenomena measured to construct the images. Two of the most widely recognizable medical tomography techniques are Computed Tomography (CT) and Magnetic Resonance Imaging (MRI). CT uses x-rays projected through the target and captured on the far side with a detector at a variety of angles. CT images show density; the X-rays are absorbed in proportion to the density of the medium. MRI uses a strong magnetic field and induced electromagnetic fields to measure the magnetic alignment of protons, mainly hydrogen nuclei in humans, within a target. This gives very high resolution images indicating the distribution of water molecules throughout a subject.

Other types of tomography include confocal laser scanning microscopy, various electrical impedance and capacitance tomography techniques, magnetic induction tomography, neutron tomography, ocean acoustic tomography (sonar), optical projection and optical coherence tomography, positron emission tomography (PET), single photon emission computed tomography (SPECT), ultrasound tomography, and seismic tomography (ground penetrating radar). These techniques use a wide array of physical sources including electromagnetic radiation in the ultrasound, radio and optical frequency ranges, and nuclear sources (i.e. x-rays, gamma rays, electron-positron annihilation).[12]

Tomography is used in an equally broad array of contexts: the medical field, geophysical investigations in the oil and gas industry, materials science and nondestructive testing in a range of manufacturing industries, and process monitoring in industrial manufacturing such as chemical plants. 
With an understanding of what tomography can be, an examination of the "electrical impedance" component of EIT gives meaning to the modality.

\subsection{Electrical Impedance}

EIT applies low frequency current to a target and measures the resulting voltages through electrodes. It measures the impedance (resistance, capacitance and inductance) throughout the domain. Unlike x-rays used in CT, the current does not travel in a straight line through the object but flows along a path dictated by the distribution of impedance. This makes reconstructing the impedance from the measurements particularly challenging and makes it difficult to obtain enough information to resolve an image at all, let alone one that would be considered "high resolution".

Despite these challenges, EIT remains a viable technology because it is portable, non-invasive, non-destructive, and inexpensive. MRI and CT systems can cost millions of dollars and require specially constructed rooms to safely house the machinery. A typical EIT system can be used bedside or transported in a small suitcase and costs thousands of dollars, orders of magnitude less than CT or MRI. Long term monitoring is a possibility because the system is small, portable and inexpensive. (Figure 2.1 and 2.2)

\section{$2.3 \quad$ Electrical Safety}

Because biomedical EIT is using current applied through a person's body as its mode of measurement, electrical safety becomes an immediate concern. The limit for how much current is acceptable to pass through the human body varies depending 


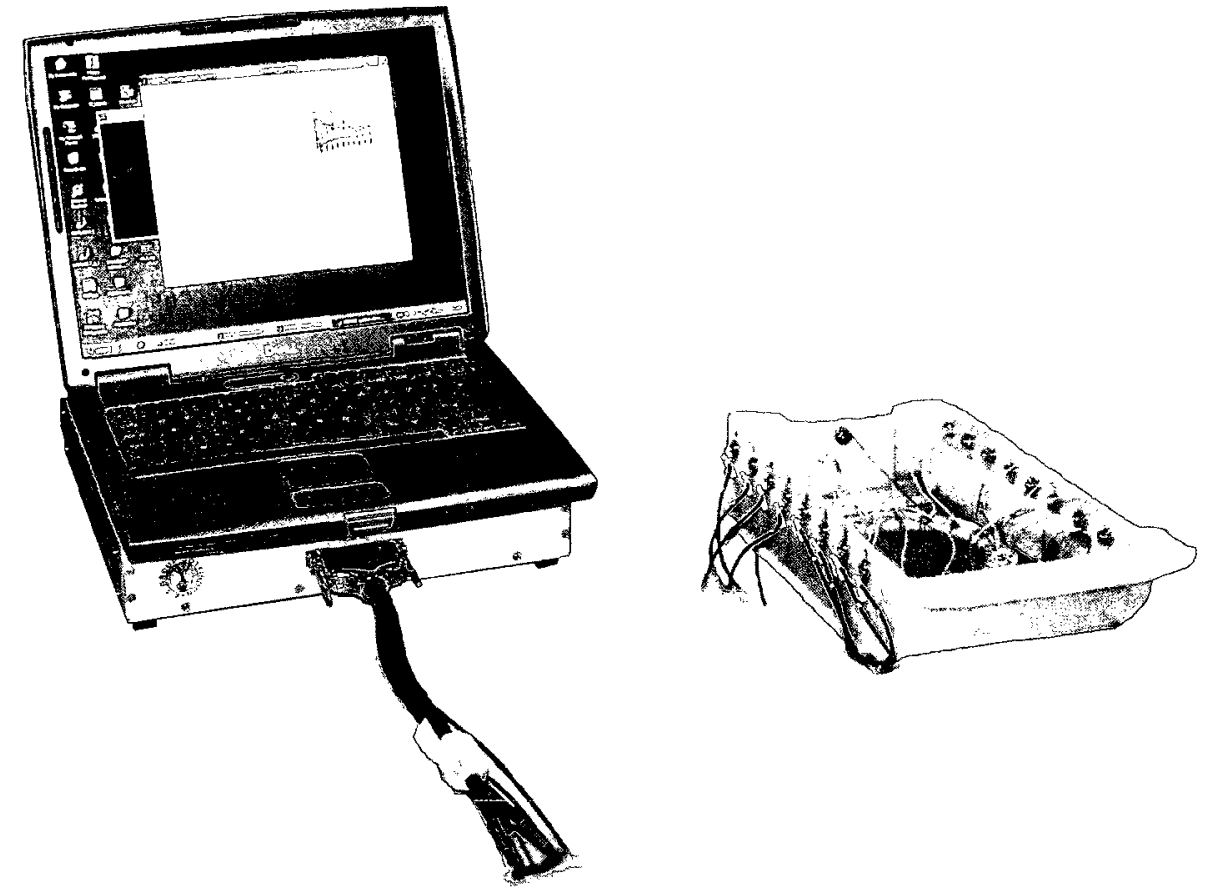

Figure 2.1: Example EIT system, photo; (left) laptop sitting on top of Goe-MF II EIT system (Viasys Healthcare, Höchberg, Germany), (right) electrode wires connecting to phantom (yellow)

on how it is applied and at what frequency. Measurements [13] have shown that as little as $10-\mathrm{mA}$ at $60-\mathrm{Hz}$ was the limit before a human could no longer release a wire when held in each hand (known as the "let-go current"). Currents above 10-mA can have serious physiological effects including respiratory paralysis, pain, and ventricular fibrillation. Beyond an Ampere, sustained myocardial contractions, burns, and other injuries occur. Below the 10-mA let-go limit, there is a region where a sensation of tingling occurs. With a further reduction in current, there is no discernible sensation. The let-go current and the corresponding thresholds for sensation fall to a minimum around a frequency of 60Hz.[14] (Figure 2.3 and 2.4)

The skin acts as an electrically protective layer around the mostly saline human 


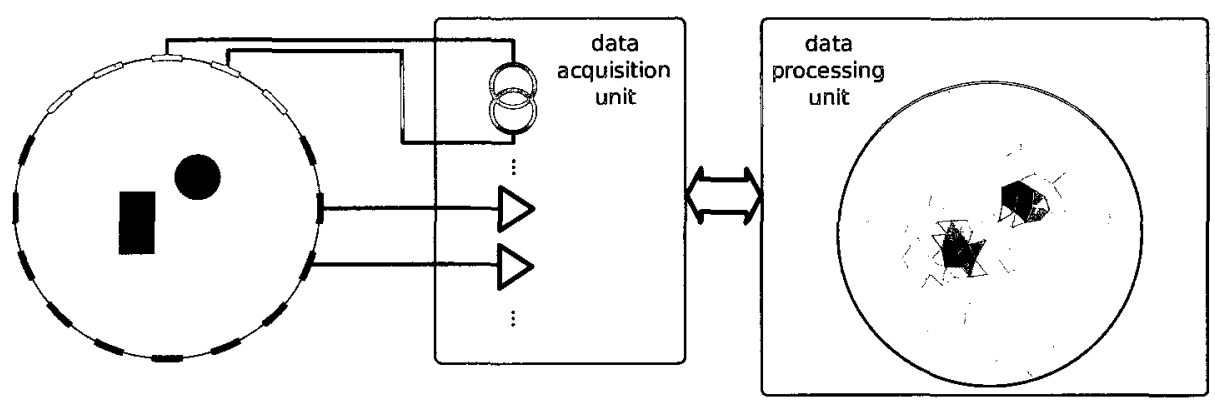

Figure 2.2: Example EIT system, schematic; (left-to-right) subject with attached electrodes, data acquisition unit including current source and voltage measurement hardware (voltage is measured relative to a ground electrode placed elsewhere on the subject), data processing unit controls data acquisition unit and handles data processing and display

body. Reduction in the resistance of this protective layer, through abrasion, the use of adhesive electrodes, or needles, significantly reduces the safe current level. The IEC 60601-1 standard [16] specifies safe current levels and leakage current limits for medical equipment operating under biomedical conditions.

Biomedical EIT typically operates with current sources running at $50 \mathrm{kHz}$ and with an upper limit of 5-mA current. These values are well within the safe operating range for biomedical equipment. In Canada, medical devices such as EIT systems intended for use with human subjects must be certified to comply with IEC 60601-1 by certified laboratories such as Underwriters Laboratories (UL) or the Canadian Standards Association (CSA) to affirm that they meet these safety standards and do not endanger researchers or subjects.

\subsection{A Brief History}

This section is a brief summary of the material contained in $[3, \S \mathrm{B}]$ which itself refers to $[17,18,19,20,21,22,23]$. 


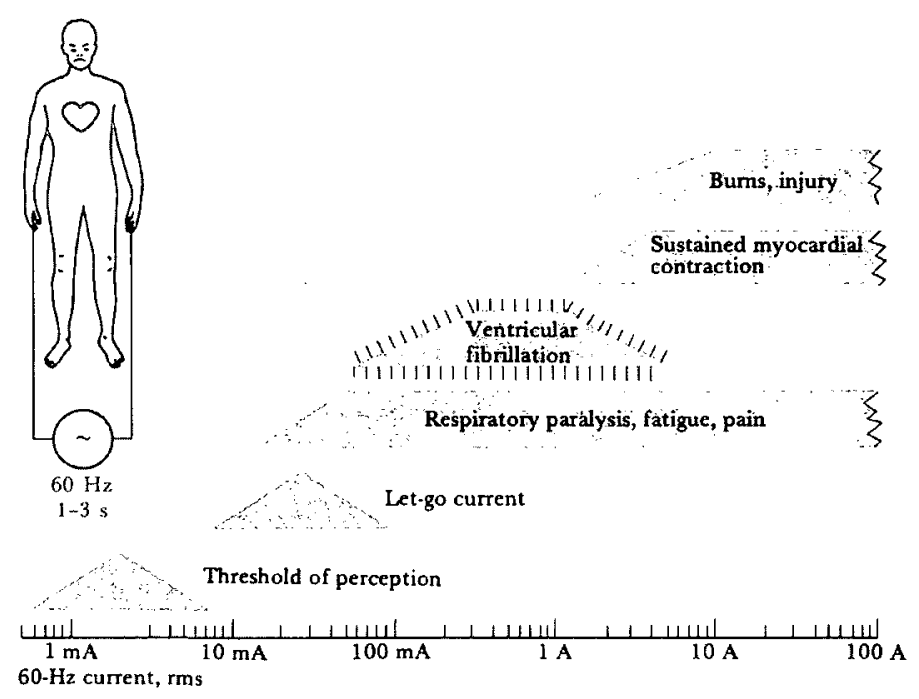

Figure 2.3: Physiological effects of electricity; threshold or estimated mean values are given for each effect in a 70-kg human for a 1- to 3-s exposure to $60-\mathrm{Hz}$ current applied via copper wires grasped by the hands. (reproduced from [14, Fig14.1])

Biomedical EIT has existed since approximately 1976, when initial images were constructed using a large number of electrodes on a single side of the chest.[17] Brain imaging for tumors was proposed shortly after with parallel electrode arrays and a saline filled tank used as a proof-of-concept.[18] The first widely used and commercially available system for what was then called Applied Potential Tomography (APT) was the Sheffield Mark I.[19] It used the, now familiar, ring of sixteen electrodes configuration. The first published images resulting from this system showed a crude cross-section of the forearm.[20,21] Other groups have continued to developed EIT systems and have taken advantage of an improved understanding of the hardware problems in EIT and the reduced size and improved quality of electronics over time. Systems have been built at various universities (Sheffield, Rensselaer Polytechnic Institute, École Polytechnique Montreal, Cardiff University, Georg-August-Universitát Góttingen, and others)[22] while commercial systems have been manufactured by 


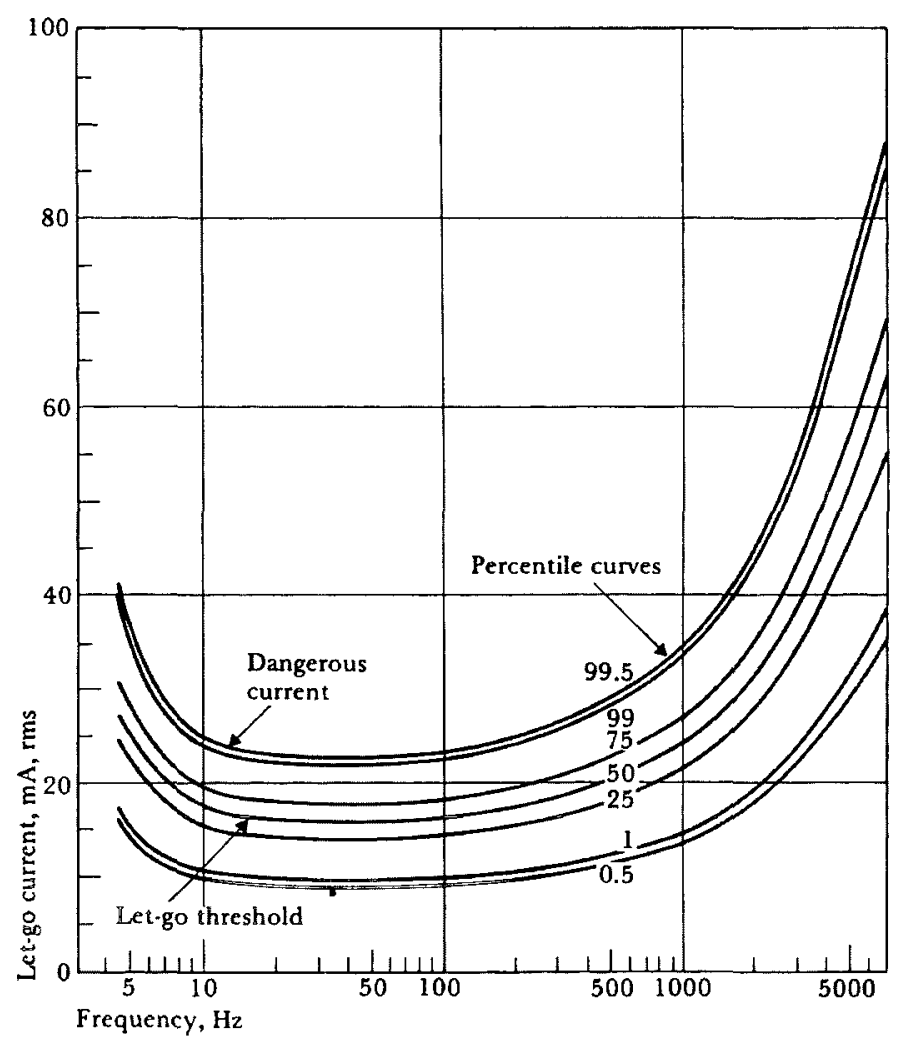

Figure 2.4: Let-go current versus frequency; percentile values indicate variability of let-go current among individuals. Let-go currents for women are about two-thirds the values of men. (reproduced from [14, Fig14.3], in-turn reproduced from [15])

CardioInspect, Draeger Medical and Viasys. EIT has not found favour within the broader medical community largely because of its low resolution when compared to ultrasound and CT. Biomedical interest has largely focused on breast cancer, the cardiac cycle, gastric emptying, brain function and pathology, and lung ventilation.[23]

More recently, research has branched out into several related fields: Magnetic Induction Tomography (MIT), Magnetic Resonance EIT (MREIT), and multifrequency EIT. MIT uses coils to induce electromagnetic fields and measure the resulting eddy currents. MREIT combines the MRI modality with simultaneous EIT to obtain detailed information on conductivity at higher resolutions. Multifrequency 
EIT applies current at a variety of frequencies to obtain information on the frequency response of the internal impedance distribution. Most systems have the capability of imaging absolute conductivities but perform difference imaging instead, finding the change in conductivity, as a means of dealing with numerous factors that otherwise introduce unacceptable artifacts into the image.

The current state of EIT can be summed up with the quote "it doesn't clearly work, so we can reap the fruits of its images, or not work, so we can change direction; it usually almost works, which is an incitement to redouble our efforts."[3]

\subsection{Breaking it Down}

The EIT problem is solved using a combination of tools. The Finite Element Method (FEM) is used to solve the forward problem, simulating the voltages on the boundary and within the domain given a set of input currents and the conductivity distribution. The inverse problem, that of discovering the conductivity distribution given currents and voltages on the boundary, requires the creation of a Jacobian to describe how the boundary voltage changes with respect to conductivity and the application of various regularization techniques to solve the problem. (Figure 2.5) The resulting image shows the conductivity distribution on the interior of the domain. (Figure 2.6) When in vivo measurements are used in the inverse problem, the forward problem's formulation is still required. 


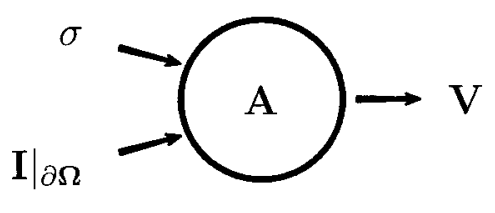

(a) forward

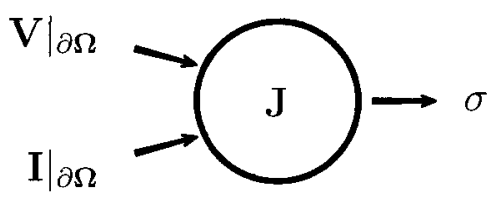

(b) inverse

Figure 2.5: Forward and inverse problem flow; forward (a) and inverse (b) problems use the variables: conductivity $\sigma$, voltage everywhere on the domain $\mathbf{V}$ and only on the boundary $\left.\mathbf{V}\right|_{\partial \boldsymbol{\Omega}}$, current on the boundary $\left.\mathbf{I}\right|_{\partial \boldsymbol{\Omega}}$, forward FEM system matrix $\mathbf{A}$, and inverse Jacobian $\mathbf{J}$

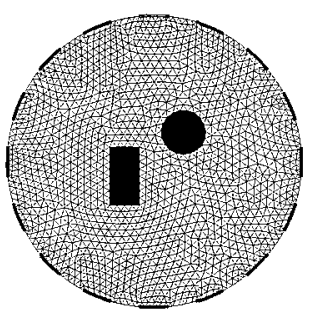

(a) forward

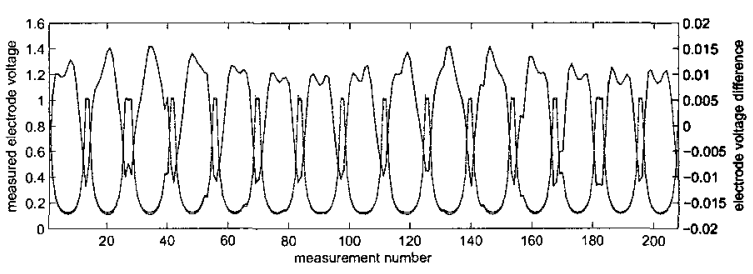

(b) voltage measurements

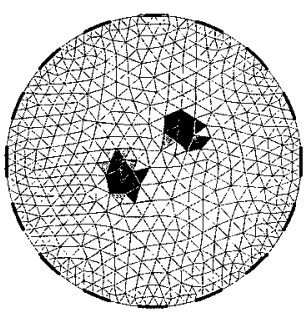

(c) inverse

Figure 2.6: Sample difference EIT simulation; forward model (a) used to generate difference voltage measurements ( $b$, red $=$ blue - green voltages) given a conductivity distribution and applied currents, measurements are then used to reconstruct a conductivity distribution (c) by solving the inverse problem 


\section{Chapter 3}

\section{The Finite Element Method}

This chapter is largely derived from $[24]$ and $[3, \S 1.8 .1]$ with additional material from [25] and [26].

Electrical Impedance Tomography (EIT) requires the use of the Finite Element Method (FEM) in both the forward and inverse problems. The FEM is a numerical technique for solving Partial Differential Equations (PDEs) by converting the problem into a system of linear equations. These linear equations are based on an approximation that breaks the domain of the problem into elements, where the connected elements are called a mesh. Each element has a portion of the solution over its region which is controlled by an expansion function that has values over the element and is zero elsewhere. The expansion function's profile over the element is, in turn, uniquely controlled by a set of node values.

Each element, in discrete form, is expressed as an element matrix that captures the solution of the problem within the element and the boundary conditions upon which the solution depends. In general, for elements that are connected, the bound- 
ary conditions of the adjoining elements will cancel. When the element matrices are assembled into a system matrix, they incorporate the underlying conditions of the problem, and the conditions on the boundary of the domain form a second vector.

In EIT, FEM models are used to solve for the voltage distribution over the domain, given a known conductivity (the underlying conditions) and the applied current at the electrodes (the boundary conditions). The solution of this forward problem allows simulation of the voltages expected at the electrodes and anywhere on the domain given a set of applied currents.

\subsection{Finding Voltage on a Domain}

In EIT, generally, the intent is to solve for the conductivity, but before getting to the inverse problem, the formulation of the forward problem is required. The derivation and corresponding assumptions for the governing equations of EIT are outlined in the following sections as well as a description of how these equations are implemented within the FEM framework.

\subsubsection{Assumptions}

The assumptions upon which single frequency EIT is based are as follows:

1. the system is quasi-static, meaning that the system is operating at a low enough frequency such that, approximately, there are no time varying components in the electric and magnetic flux density fields,

$$
\omega \simeq 0 \quad \frac{\partial \mathbf{D}}{\partial t} \simeq 0 \quad \frac{\partial \mathbf{B}}{\partial t} \simeq 0
$$


2. the medium behaves as a linear conductor, such that current flow is distributed through the medium dependent solely on conductivity and induced electric field,

3. the medium is dispersionless, such that there are no losses due to heating or other means, and

4. the electric field $\mathbf{E}$ is $C^{2}$ smooth (i.e. it has first- and second-derivatives), and the field tends to zero at a rate faster than $1 / r^{2}$ as the distance $r$ approaches infinity.

Current is represented by a complex phasor at a frequency of interest. Where that frequency is low, assumption (1) indicates that any frequency dependent susceptibility component $\varepsilon$ of admittivity $\gamma$ is assumed to have no effect since $\omega \simeq 0$.

$$
\gamma=\sigma+\mathrm{i} \omega \varepsilon \quad \stackrel{\omega \simeq 0}{\longrightarrow} \quad \gamma \simeq \sigma
$$

Thus, conductivity $\sigma$ has only a real component but is not constrained to be isotropic and therefore, may be a scalar or a matrix.

Assumption (4) is required for application of the Helmholtz decomposition and is satisfied for any real world electrostatic case but can be violated in contrived problems.

\subsubsection{Derivation}

The equation governing the behaviour of EIT systems is founded in fundamental electromagnetics. By taking the aforementioned assumptions and applying some 
algebraic manipulation, the desired equation can be derived. This in itself is not enough, however; further massaging is required to make these equations usable in a FEM setting by transforming the system of discrete equations into functions of first derivatives.

The derivation of the governing equations for EIT begins with Maxwell's equations for electromagnetics

$$
\begin{array}{rrr}
\nabla \cdot \mathbf{D} & =\rho / \epsilon_{0} & \text { Gauss' Law } \\
\nabla \cdot \mathbf{B} & =0 & \text { Gauss' Law for electromagnetism } \\
\nabla \times \mathbf{E} & =-\frac{\partial \mathbf{B}}{\partial t} & \text { Faraday's Law } \\
\nabla \times \mathbf{H} & =\mu_{0} \epsilon_{0} \frac{\partial \mathbf{D}}{\partial t}+\mu_{0} J & \text { Ampere's Law }
\end{array}
$$

Employing the quasi-static assumption where $\partial \mathbf{B} / \partial t \simeq 0$ (3.1) and applying it to Faraday's Law (3.3c) gives

$$
\nabla \times \mathbf{E} \simeq 0
$$

This indicates that assuming a quasi-static field means the field is "irrotational," and by applying the Helmholtz decomposition, the electric field $\mathbf{E}$ is seen to be the gradient of a scalar function

$$
\mathbf{E} \simeq-\nabla \phi
$$

where $\phi$ is the potential.

Taking the conservation of charge law (the divergence of curl identity),

$$
\nabla \cdot(\nabla \times \mathbf{H})=0
$$


and substituting in Ampere's law (3.3d) gives

$$
\nabla \cdot\left(\mu_{0} \epsilon_{0} \frac{\partial \mathbf{D}}{\partial t}+\mu_{0} J\right)=0
$$

and then, employing the quasi-static assumption where $\partial \mathbf{D} / \partial t \simeq 0(3.1)$ gives

$$
\nabla \cdot\left(\mu_{0} J\right) \simeq 0
$$

where rolling the permeability of free space constant into the current density $\mu_{0} J \rightarrow J$ gives

$$
\nabla \cdot J \simeq 0
$$

Kirchhoff's formulation of Ohm's law assumes a conductor operating in a linear range with no dispersion

$$
J=\sigma \mathrm{E}
$$

Substituting this into (3.9) and applying our equation for a quasi-static electric field (3.5) gives

$$
\begin{array}{r}
\nabla \cdot(\sigma \mathbf{E}) \simeq 0 \\
\nabla \cdot(\sigma \nabla \phi) \simeq 0
\end{array}
$$

which is a generalized Laplace equation that describes the relationship between conductivity and potential over a domain. It is an elliptic PDE whose solution is a harmonic function. (Harmonic functions are twice continuously differentiable and satisfy Laplace's equation.) Analytic solutions for this equation can be obtained for 
simple geometries and conductivity distributions, but for more complicated configurations, numerical solutions can be found using a FEM model with considerably less difficulty.

\subsection{Weak Form}

To resolve the governing equation for EIT over a domain, the governing equation must first be converted to a different form so that it can be solved numerically. The FEM solution of (3.12) requires that the equation be converted to its weak form by applying Stokes' Theorem to convert the original equation containing a secondorder derivative into an equation containing only first-order derivatives. To apply Stokes' Theorem, the surface must be orientable and smooth. A surface is orientable if the surface normal is well defined, as it is for most physical objects, allowing the right-hand rule to be specified. A Möbius strip is an example of an object with an undefined surface normal. An object is smooth if it is differentiable (i.e. has no singularities or discontinuities). For (3.12), the surface needs to be $C^{2}$ smooth (i.e. has first- and second-order derivatives).

The weighted residual is used to obtain a solution to the FEM model such that the integral is approximately zero when some test function $u$ is applied

$$
\nabla \cdot(\sigma \nabla \phi)=0 \quad \rightarrow \quad \int_{\Omega} u(\nabla \cdot(\sigma \nabla \phi)) d V=0
$$

By taking the vector derivative identity and applying it to the EIT governing equation, the second-order derivative is separated into two new terms, one of which has 
only first-order derivatives.

$$
\begin{aligned}
\nabla \cdot(f A) & =f(\nabla \cdot A)+(\nabla f) \cdot A \quad \text { where } f=u, \quad A=\sigma \nabla \phi \\
\nabla \cdot(u \sigma \nabla \phi) & =u(\nabla \cdot(\sigma \nabla \phi))+\underbrace{\nabla u \cdot(\sigma \nabla \phi)}_{\sigma \nabla u \cdot \nabla \phi}
\end{aligned}
$$

Rearranging (3.15) for the first term on the right-hand side and substituting into (3.13) gives a weighted residual function

$$
\int_{\Omega} \nabla \cdot(u \sigma \nabla \phi) d V-\int_{\Omega} \sigma \nabla u \cdot \nabla \phi d V=0
$$

Applying the divergence theorem to the left-hand term of (3.16) gives

$$
\int_{\Omega}(\nabla \cdot F) d V=\oint_{\partial \Omega} F \cdot \hat{n} d S \quad \rightarrow \quad \int_{\Omega} \nabla \cdot(u \sigma \nabla \phi) d V=\oint_{\partial \Omega}(u \sigma \nabla \phi) \cdot \hat{n} d S
$$

which is the weak form, where the second-derivative of potential $\phi$ in (3.13) has been reduced to a first-derivative by transferring a derivative to the test function $u$

$$
\int_{\Omega} \sigma \nabla \phi \cdot \nabla u d V=\oint_{\partial \Omega}(u \sigma \nabla \phi) \cdot \hat{n} d S
$$

The potential gradient term normal to the boundary $\nabla \phi \cdot \hat{n}$ is the outward normal current density on the boundary $(J \cdot \hat{n})$, and the current density on the boundary is zero everywhere except where electrodes are attached. 


\subsection{Expansion Functions}

A test function similar to the one used in the previous section is required to obtain a discrete approximation of the potential $\phi$. These test functions can be chosen arbitrarily. The Galerkin method, or more specifically the Rayleigh-Ritz method, elects to use the same discretization for the two test functions. The discretization functions are called the expansion functions, where $u=\Psi, \phi(x)=\sum_{i} \phi_{i} \Psi_{i}(x)$.

The choice of expansion function used for the discretization of the potential distribution affects the distribution that can be accurately approximated. To make interpretation of the results simple, it is desirable that first-order expansion functions have a value of one at a single node of the element, zero at the other nodes, and zero outside of the element. When multiplied by node values, the summation of expansion functions for an element will give the value of the approximation at a point within the element. At the exact location of a node, the approximation is independent of other nodes thereby simplifying the process of plotting the solution. The summation of all elements will give an approximation of the function over the domain. A firstorder scalar interpolatory expansion function, a straight line between node values, is the simplest starting point allowing for zero-order continuity $C^{0}$ between elements. (Figure 3.1) Expansion function choices include hierarchical interpolatory, Legendre,

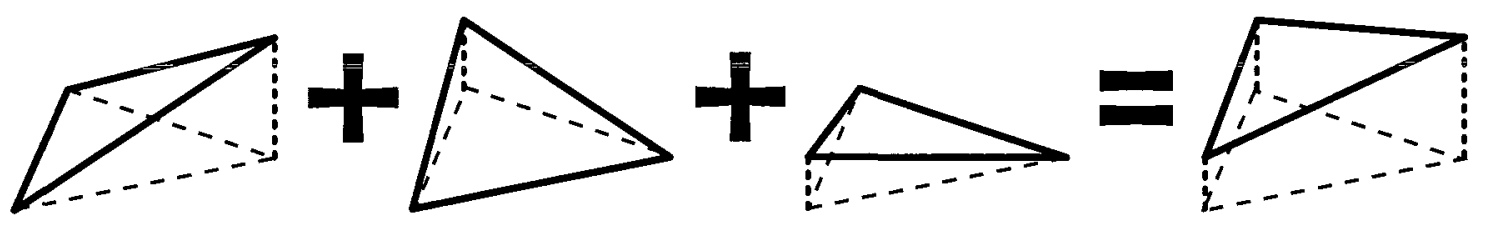

Figure 3.1: Two-dimensional first-order scalar interpolatory expansion functions; sum of expansion functions controlled by the mesh element's three corner nodes gives a linear approximation over the element 
Hermite, and other specialized polynomials that allow second and higher order curves between nodes in the discretization. These additional degrees of freedom can allow manipulation of the expansion functions to enforce physical constraints, such as enforcing continuity when assembling the system matrix or to isolate components of the expansion function in other ways. (Figure 3.2) Increasing the polynomial order of the discretization, in FEM terminology, is called $p$-refinement and can be done on an element-by-element basis. Splitting individual elements into smaller elements is called $h$-refinement. Typically, commercial FEM implementations would form an initial solution on a basic mesh and then refine the mesh based on some estimate of the discretization error.

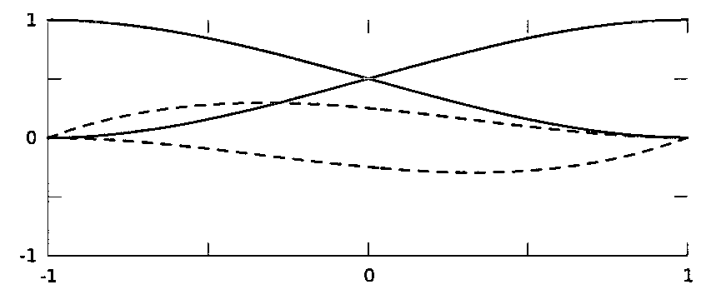

(a) 1st- and 2nd-order Hermite functions

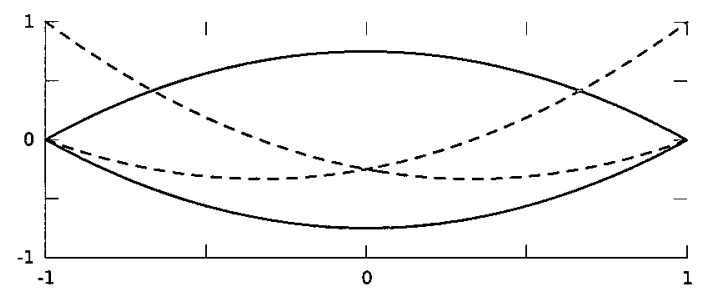

(b) Hermite derivatives

Figure 3.2: An example of an alternative expansion function; one-dimensional firstorder (solid) and second-order (dashed) Hermite expansion functions (a) and their derivatives (b); note that the value of the second-order expansion functions at the edges of the element $(+1$ and -1$)$ are zero, while the derivatives of the first-order expansion function are zero at those same points allowing independent control of slope and value at the node

In general, linear expansion functions with no refinement are the most common choice for EIT. The conductivity is generally treated as piecewise scalar constant (i.e. a constant across each element of the mesh) though first-order linear approximations have also been used.[3, 26] 
The element equations take the form, for each $i$

$$
\sum_{j} \phi_{j} \int_{\Omega} \sigma \nabla \Psi_{i} \cdot \nabla \Psi_{j} d V-\oint_{\partial \Omega}(u \sigma \nabla \phi) \cdot \hat{n} d S=0
$$

where the test function and continuous potential have been substituted for their discrete equivalents using the common expansion function $\Psi$. Potential at the node $j$ is a scalar constant $\phi_{j}$. The element equations can be written in matrix form as the element matrices

$$
\mathbf{A}^{e} \mathbf{x}^{e}=\mathbf{b}^{e}
$$

where

$$
\begin{aligned}
\mathbf{A}_{i j}^{e} & =\int_{\Omega} \sigma \nabla \Psi_{i} \cdot \nabla \Psi_{j} d V \\
\mathbf{b}_{i}^{e} & =\oint_{\partial \Omega}(u \sigma \nabla \phi) \cdot \hat{n} d S \\
\mathbf{x}_{j}^{e} & =\phi_{j}
\end{aligned}
$$

The row and column of the matrix are identified by $i$ and $j$ respectively. For a firstorder scalar interpolatory expansion function in two dimensions with triangular mesh elements, there are three expansion functions $i, j=1 . .3$ forming a 3 -by-3 element matrix $\mathbf{A}^{\mathrm{e}}$.

Combining the summation of the element matrices over all nodes gives the system matrix, where the boundary conditions $\mathbf{b}^{e}$ for adjoining elements cancel and leave only the boundary conditions on the surface of the domain.

The system matrix takes the form of a system of linear equations representing a circuit $V=I R$, and this interpretation leads to a second way of viewing the meshed 
domain. The FEM mesh can be viewed as a resistor model where resistors connect the nodes instead of a continuous domain of conductivity. Boundary conditions can be interpreted as a current source attached to a node for the Point Electrode Model (PEM), a shunt connecting each electrode's nodes for the Shunt Electrode Model (SEM), or an additional node in the resistor network with impedances connecting to the electrode's nodes for the Complete Electrode Model (CEM).

\subsection{Boundary Conditions}

In FEM terminology, there are two types of boundary conditions: "Dirichlet" and "Neumann" boundary conditions. Dirichlet boundary conditions directly assign a value to the discretized function on the boundary. In EIT, this would be assigning a specific potential $\phi$ to a node. A Neumann boundary condition sets the first derivative of the discretized function in the normal direction to the boundary. (The normal is usually an "outward" unit normal; though it depends on the "right-handedness" of the domain, whether the right-hand rule has been applied in assigning the vector components of the domain.) For EIT, this would be a current density $J$ into the domain over an electrode $\sigma \partial \phi / \partial \hat{n}=J$. Mixed boundary conditions are also possible combining the Neumann and Dirichlet boundary conditions.

For a PEM, the boundary conditions are applied by setting the appropriate input or output current $I$ in the system matrix. This does not directly represent a current density over an element but instead, requires interpretation of the FEM mesh as a resistor network.

SEMs require an additional step with respect to the PEM which is to set all nodes 
associated with an electrode to the same voltage. This is achieved by "forcing" the matrix by replacing rows and columns in the system matrix for all but one of the nodes so that they will be assigned the same voltage (i.e. $\phi_{1}=\phi_{2}$ ). The remaining node is assigned the input or output current which will be distributed across the shunt.

For a more realistic boundary condition, a CEM can be implemented as a "Neumann" boundary condition. This implies an additional contact impedance for each electrode which is distributed across the boundary associated with that electrode. The simplest approximation is to assume a uniform distribution of contact impedance over the area of the electrode.

$$
\sigma \frac{\partial \phi}{\partial \hat{n}}= \begin{cases}J & \text { electrodes } \\ 0 & \text { elsewhere }\end{cases}
$$

Taking the integral of (3.22) gives the current through the electrode $\int J d S=I$.

The system matrix with the CEM for all electrodes then becomes

$$
\left[\begin{array}{cc}
\mathbf{A}_{\alpha}+\mathbf{A}_{\beta} & \mathbf{A}_{\gamma} \\
\mathbf{A}_{\gamma}^{\top} & \mathbf{A}_{\delta}
\end{array}\right]\left[\begin{array}{l}
\mathbf{x}_{n} \\
\mathbf{x}_{k}
\end{array}\right]=\left[\begin{array}{l}
0 \\
\mathbf{b}
\end{array}\right]
$$

where $\mathbf{x}_{n}$ is the voltage on the FEM mesh's nodes, $\mathbf{x}_{k}$ is the voltage measured at the 
electrodes with outward current flow $\mathbf{b}$, and

$$
\begin{aligned}
\mathbf{A}_{\alpha i j} & =\sigma \int_{\Omega} \nabla \Psi_{i} \cdot \nabla \Psi_{j} d V \\
\mathbf{A}_{\beta i j} & =\sum_{k} \frac{1}{z_{k}} \int_{E_{k}} \Psi_{i} \Psi_{j} d S, \quad i, j \in E_{k} \\
\mathbf{A}_{\gamma i k} & =\frac{1}{z_{k}} \int_{E_{k}} \Psi_{k} d S, \quad i \in E_{k} \\
\mathbf{A}_{\delta k k} & = \begin{cases}1 & k \in E_{k} \\
0 & \text { elsewhere }\end{cases}
\end{aligned}
$$

where an electrode $k$ has a set of nodes $E_{k}$ associated with it. The sub-matrix $\mathbf{A}_{\delta}$ is an identity matrix I. As before, the row and column of the matrix are identified by $i$ and $j$ respectively, and the rows and columns associated with $k$ are those that augment the matrix to implement the CEM. Note that integrals over the expansion functions and their first derivatives, dependent on the geometry of the elements, are required. If the mesh does not change, the integrals over the expansion functions can be precomputed.

This finite element formulation, with first-order interpolatory expansion functions, is implemented in the Electrical Impedance and Diffuse Optics Reconstruction Software (EIDORS) package, version 3.3.

Equations (3.24) form the setting in which EIT is performed, but in fact, it is not the final problem to be solved. The problem when stated this way does not match the information available: a set of applied currents and measured voltages on the boundary with an unknown conductivity. To find the unknown conductivity requires a further tool: inverse problem theory. 


\section{Chapter 4}

\section{Inverse Problems}

This chapter is derived from $[27,28]$ and $[3, \S 1.1-1.7]$ with additional material from [25] and [26].

Inverse problems are those problems where parameters must be inferred from a set of data. In Electrical Impedance Tomography (EIT), the problem is inferring the interior conductivity from applied currents and the resulting voltages on the boundary. This is not the direct opposite of the forward problem (voltages found everywhere from injected current and known conductivity) but instead, a slightly different problem where the currents are assumed constant and conductivity is reconstructed by examining how the measured voltages would change if conductivities changed slightly.

According to the mathematician Jacques Hadamard (1865-1963), mathematical problems can be classified as either "well-posed" or "ill-posed". The Hadamard criteria for a well-posed problem are as follows:

1. for all admissible data, a solution exists, 
2. for all admissible data, the solution is unique, and

3. that the solution depends continuously on the data.[29]

In EIT, the existence and uniqueness of a solution, conditions 1 and 2, have been proven for some specific cases where the boundary takes a simple cylindrical form and interior conductivity is severely constrained: with all boundary data known, with gaps in the data, and with various electrode models. [30, 31, 32, 33] Typically, analytic analysis of more complex boundaries and interior conductivities rapidly becomes infeasible without resorting to broad approximations. The real challenge in EIT comes from condition 3 (that the solution should depend continuously on the data). The problem is that small changes in the measurements correlate with significant conductivity changes in the interior because changes in the applied current affect all measurements; the problem is non-local. As a result, the addition of noise to the measurements can result in significant changes in the resulting reconstructed image.

The solution of EIT images is formulated using the Finite Element Method (FEM) discretization. Because the problem is discrete, it is more properly referred to as an ill-conditioned problem.[27]

\subsection{Finding Conductivity Change}

Understanding the forward problem, from the previous chapter, of finding voltages everywhere from conductivity and applied current, the inverse problem in EIT is defined as finding an operator that converts the measurement data into a conductivity. (Recall that $\mathbf{A x}=\mathbf{b}$, where $\mathbf{x}$ are node voltages from the FEM and $\mathbf{b}$ are the bound- 
ary currents.) For difference imaging, the absolute voltages and conductivities are not of interest, but instead, their respective changes $\Lambda_{\sigma}:\left.\Delta \phi\right|_{\partial \Omega} \mapsto \Delta \sigma$ are of interest. With a linearized discrete approximation of the domain, the Dirichlet-to-Neumann operator $\Lambda_{\sigma}$ is a Jacobian $\mathbf{J}$, a matrix of all first-order partial derivatives of measured voltages with respect to conductivity. It is recognized as a transconductance operator and is sometimes called the sensitivity matrix.

The standard method of deriving the Jacobian from the forward problem uses an extraction matrix $\mathbf{T}$ on the forward solution's voltages $\mathbf{x}$ to select the nodes of the FEM mesh to use as the measured voltage $\mathbf{v}$. Since the problem is to find a difference image, only the change in voltages at the electrodes is of interest.

$$
\mathbf{z}=\Delta \mathrm{V}=\mathbb{T}\left[\mathbf{x}\left(\sigma_{2}\right)-\mathbf{x}\left(\sigma_{1}\right)\right]=\mathbb{T}\left[\mathbf{x}\left(\sigma_{\mathfrak{0}}+\Delta \sigma\right)-\mathbf{x}\left(\sigma_{0}\right)\right]
$$

Multiplying top and bottom by this change in conductivity $\Delta \sigma$

$$
\mathbf{z}=\mathbf{T}\left[\frac{\mathbf{x}\left(\sigma_{0}+\Delta \sigma\right)-\mathbf{x}\left(\sigma_{0}\right)}{\Delta \sigma} \Delta \sigma\right]
$$

and taking the limit as this change approaches zero

$$
\lim _{\Delta \sigma \rightarrow 0} \frac{\mathbf{x}\left(\sigma_{0}+\Delta \sigma\right)-\mathbf{x}\left(\sigma_{0}\right)}{\Delta \sigma} \simeq \frac{\partial \mathbf{x}\left(\sigma_{0}\right)}{\partial \sigma}
$$

gives the partial derivative. Substituting this back into the difference voltage equation (4.1), with $\mathbf{c}=\Delta \sigma$, gives

$$
\mathbf{z}=\mathbf{T}\left[\frac{\partial \mathbf{x}\left(\sigma_{0}\right)}{\partial \sigma} \mathbf{c}\right] \quad \text { where } \quad \mathbf{x}\left(\sigma_{0}\right)=\mathbf{A}\left(\sigma_{0}\right)^{-1} \mathbf{b} \quad \rightarrow \quad \mathbf{x}_{0}=\mathbf{A}_{0}{ }^{-1} \mathbf{b}
$$


and the partial derivative is used with a change in notation to identify that the FEM matrix $\mathbb{A}_{0}$ is calculated at a specific background conductivity $\sigma_{0}$.

$$
\mathbf{z}=\mathbf{T}\left[\frac{\partial\left(\mathbf{A}_{0}^{-1}\right)}{\partial \sigma} \mathbf{b c}\right]
$$

By applying the matrix derivative identity $\partial\left(\mathbf{X}^{-\mathbf{1}}\right)=-\mathbf{X}^{-1}(\partial \mathbf{X}) \mathbf{X}^{-\mathbf{1}}$ to the partial derivative

$$
\begin{aligned}
& \mathbf{z}=-\mathbf{T}\left[\mathbf{A}_{0}{ }^{-1} \frac{\partial \mathbf{A}_{0}}{\partial \sigma} \mathbf{A}_{0}{ }^{-1} \mathbf{b c}\right], \quad \mathbf{x}_{0}=\mathbf{A}_{0}{ }^{-1} \mathbf{b} \\
& \mathbf{J}=-\mathbf{T}\left[\mathbf{A}_{0}{ }^{-1} \frac{\partial \mathbf{A}_{0}}{\partial \sigma} \mathbf{x}_{0}\right]
\end{aligned}
$$

This equation can be solved as a least squares problem to find the change in conductivity $\mathbf{c}$ with

$$
\arg \min _{\mathbf{c}} i \mid \mathbf{J} \mathbf{c}-\mathbf{z} \|
$$

but is severely ill-conditioned in the presence of noise, and thus, further regularization steps are required to obtain a reasonable solution.

The standard method, outlined here, can be used to calculate the Jacobian but requires the calculation of the inverse $\mathbf{A}_{0}{ }^{-1}$ and partial derivative $\partial \mathbf{A}_{0} / \partial \sigma$ of the system matrix along with the node voltages $\mathbf{x}_{0}$. Other methods are available, including various perturbation techniques that approximate the Jacobian by simulating small changes in the conductivity, where small changes are large enough to avoid numerical precision errors while still giving a reasonably accurate solution. Many of the methods are significantly more efficient, but some approximations can be inappropriate, so an alternative must be selected with care. $[34,35,36,1]$ 


\subsection{Regularization Methods}

There is a large variety of regularization methods which can be employed to "stabilize" the solution of the inverse EIT problem. These can broadly be separated into methods that provide a solution in a single step and those that require an iterative solution process. Considering these solutions with a probabilistic framework in mind is useful when considering the effect of regularization, whatever its form.

\subsubsection{A Bayesian View}

It is helpful to consider these regularization techniques in a Bayesian framework where the a priori knowledge is clearly separated from the measurements and fixed qualities of the problem. All variables are treated as random variables with their respective probability distributions. Bayes formula

$$
P(\sigma \mid \phi)=\frac{P(\phi \mid \sigma) P(\sigma)}{P(\phi)}
$$

shows the posterior probability $P(\sigma \mid \phi)$, the probability of $\sigma$ given $\phi$, is related to the conditional probability $P(\phi \mid \sigma)$ and the prior probability $P(\sigma)$. The marginal probability $P(\phi)$ is not relevant when the posterior probability does not need to be normalized. An example is the Maximum A Posteriori (MAP) estimate where the solution is

$$
\hat{\sigma}_{M A P}=\arg \max _{\sigma} P(\sigma \mid \phi)
$$


the maxima of the posterior probability. The Conditional Mean (CM) estimate is another Bayesian solution

$$
\hat{\sigma}_{C M}=E\{\sigma \mid \phi\}=\int \sigma P(\sigma \mid \phi)
$$

which gives the mean value of the posterior distribution which is not necessarily the same as the MAP estimate particularly when the posterior distribution has more than one peak. The Maximum Likelihood (ML) estimate

$$
\hat{\sigma}_{M L}=\arg \max _{\sigma} P(\phi \mid \sigma)
$$

asks "What is the most likely value $\sigma$ to have produced this outcome $\phi$ ?" It is possibly the most common statistical estimate but frequently corresponds to solving the unregularized solution.

The Bayesian interpretation shows that there is always some quantity of prior knowledge incorporated into a reconstruction and rarely, are there any certainties involved in either the measurements or the solution. With regularization techniques that do not appear to incorporate prior information, it is not obvious what assumptions are being made. For example, with difference imaging in EIT, a prior of zero conductivity change across the domain is frequently being implicitly assumed. Another common prior assumption is one of smoothness on the conductivity distribution. Likewise, a single reconstructed image represents only a single selection from the posterior distribution. Regularized reconstruction algorithms bias that selection towards prior assumptions, but without detailed analysis, there is no guarantee that the posterior distribution has a single maximum (i.e. there may be similar conduc- 
tivity images that result in the same measurements). There is no perfect solution for a given set of measurements when there is noise in those measurements due to the instability of the inverse problem.

A desirable property for any regularization mechanism is that there be a clear separation between the a priori information, the measurement data and the regularization mechanism.

\subsubsection{Single Step Solutions}

The simplest reconstruction algorithm for the problem $\mathbf{J c}=\mathbf{z}$ would be one using the inverse of $\mathbf{J}$ such that $\mathbf{c}=\mathbf{J}^{-1} \mathbf{z}$. This will not work for EIT problems because the matrix $\Xi$ is not square and therefore, does not have an inverse. A simple solution is to use the Moore-Penrose pseudoinverse $\mathbf{J}^{\dagger}=\left(\mathbf{J}^{*} \mathbf{J}\right)^{-1} \mathbf{J}^{*}$ where the columns of $\mathbf{J}$ are linearly independent. This is the least squares solution $\mathbf{c}_{l s}=\arg \min _{\mathbf{c}}\|\mathbf{J c}-\mathbf{z}\|^{2}=$ $\mathbf{J}^{\dagger} \mathbf{z}$ given by the backslash operator $(\mathbf{J} \backslash \mathbf{z})$ in many scientific computing environments (MatLab, Octave, SciLab, etc.).

This will not give a satisfactory solution because of the instability of the EIT problem as observed by looking at the condition number of the Jacobian $\mathbf{J}$ such that $\kappa(\mathbf{J})=\|\mathbf{J}\| \cdot\left\|\mathbf{J}^{-1}\right\|$. The condition number can be understood as the ratio of the smallest to largest singular values of the Singular Value Decomposition (SVD). The singular values of an ill-conditioned problem tend to fall off sharply with a significant number of small values. When the inverse problem is considered with the addition of noise, these small singular values are affected by the noise resulting in large fluctuations in the solution. (Figure 4.1) A small condition number indicates that an inversion will magnify any errors in the data. EIT problems typically have very 
small condition numbers unless some form of regularization is applied. To improve the stability of the solution, additional information can be added to the matrices to reduce the effect of the small singular values. This is the essence of regularization. An intuitive fix for these small singular values is the Truncated SVD (TSVD) which sets a certain number of the smallest values to zero. Unfortunately, the TSVD does not give good results in many situations, and more complex algorithms are called for.

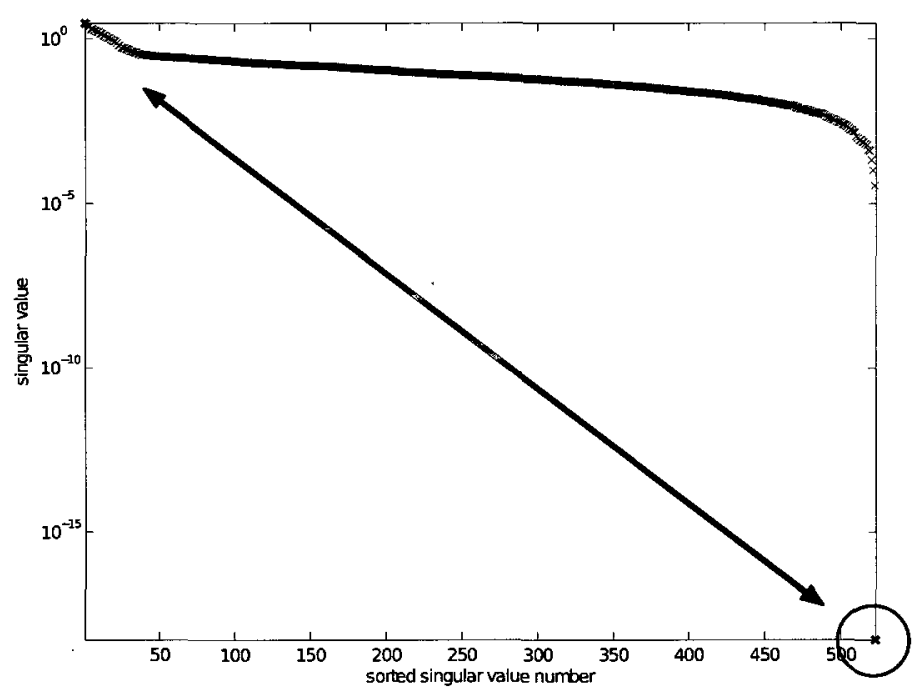

Figure 4.1: Singular values of an inverse EIT problem shown in Figure 2.6(c); 523 singular values organized from largest to smallest; note the smallest values are 17 orders of magnitude smaller than the largest values and match the machine precision

Tikhonov regularization is a basic form of regularization that admits many variations and forms a good framework for examining a variety of priors.

$$
\mathbf{c}_{\lambda}=\arg \min _{\mathbf{c}}\|\mathbf{J} \mathbf{c}-\mathbf{z}\|^{2}+\lambda^{2}\|\mathbf{c}\|^{2}
$$


which has the solution

$$
\mathbb{C}_{\lambda}=\left(\mathbb{J}^{\top} \mathbb{J}+\lambda^{2} \mathbb{I}\right)^{-1} \mathbb{J}^{\top} \mathbb{Z}
$$

where $\lambda$ is the hyper-parameter which controls the intensity of regularization.[37, 27, 35] Observe that as the hyper-parameter $\lambda$ approaches zero, reducing the amount of regularization, the solution becomes the pseudoinverse. Increasing the hyperparameter affects the condition number and admits a more stable solution.

There are three modifications that improve the broad applicability of Tikhonov regularization:

1. allow weighting of the relationship between solution values $\mathbf{L}$,

2. allow a prior solution $\mathbb{c}_{0}$ to be preferred, and

3. allow weighting of the data $\mathbf{W}$ so that unreliable data can be discounted.

This gives the generalized form of Tikhonov regularization

$$
\mathbf{c}_{\lambda}=\arg \min _{\mathbf{c}}\|\mathbf{J} \mathbf{c}-\mathbf{z}\|_{\mathbf{W}}^{2}+\lambda^{2}\left\|\mathbf{L}\left(\mathbf{c}-\mathbf{c}_{0}\right)\right\|^{2}
$$

which has the solution

$$
\begin{gathered}
\mathbf{c}_{\lambda}=\left(\mathbf{J}^{\top} \mathbf{W} \mathbf{J}+\lambda^{2} \mathbf{L}^{\top} \mathbf{L}\right)^{-1}\left(\mathbf{J}^{\top} \mathbf{W} \mathbf{z}+\lambda^{2} \mathbf{L}^{\top} \mathbf{L} \mathbf{c}_{0}\right), \text { or } \\
\mathbf{c}_{\lambda}=\left(\mathbf{L}^{\top} \mathbf{L}\right)^{-1} \mathbf{J}^{\top}\left(\mathbf{J}\left(\mathbf{L}^{\top} \mathbf{L}\right)^{-1} \mathbf{J}^{\top}+\lambda^{2} \mathbf{W}^{-1}\right)^{-1}\left(\mathbf{z}-\mathbf{J} \mathbf{c}_{0}\right)+\mathbf{c}_{0}
\end{gathered}
$$

where the latter equation (4.17) is the Wiener filter form $\mathbf{J}^{*} /\left(\mathbf{J} \mathbf{J}^{*}+\mathrm{SNR}\right)$. See Appendix B for derivations of these Tikhonov solutions (4.14) (4.16) (4.17). Setting $\mathbf{L}$ to a discrete derivative approximation creates a smoothing norm that penalizes 
sudden changes in slope, preferring "smooth" solutions. Setting $\mathbf{L}=\mathbf{I}$ gives the basic Tikhonov regularization, preferring "small" solutions that are near the image prior. This single step solution framework is implemented in the Electrical Impedance and Diffuse Optics Reconstruction Software (EIDORS) package, version 3.3.

There are a variety of regularization techniques that can be shown to be a constrained form of Tikhonov regularization such as the TSVD. Note that, with a bit of manipulation, the Bayesian MAP estimate can be reformulated as Tikhonov regularization where the covariance matrices for the measurement and regularization terms can be transformed to the Tikhonov variables $\mathbf{L}$ and $\mathbf{W}$; thus, philosophically tying the Tikhonov and Bayesian frameworks together.

\subsubsection{Iterative Solutions}

Iterative solutions can provide a means to solve problems insoluble with a single step solution or provide a significant improvement in calculation efficiency.

Non-linear Landweber, the modified Newton-Raphson method (also known as the Gauss-Newton method), the Levenburg-Marquardt method (Gauss-Newton with Tikhonov type regularization), and various flavours of non-linear conjugate gradient algorithms allow the solution of non-linear problems, such as EIT, through iterative updates of the operational linearization. [38]

Total Variation (TV) applies a regularization term that is not differentiable, and therefore, iterative solutions are required. The Primal-Dual Interior Point Method (PD-IPM) is one such technique specific to TV regularization.

Conjugate gradient and its many variants provide an efficient iterative solution to the Tikhonov functional. They provide a variety of trade-offs in efficiency versus 
accuracy, and a number of parameters, such as stopping criteria or iteration limits, which must be predetermined.

Other options include statistical methods such as Markov Chain Monte Carlo (MCMC) and multigrid techniques. [39, 40]

However, this thesis focuses on single step solutions. Some iterative techniques such as PD-IPM are implemented in the EIDORS package, version 3.3.

\subsection{Hyper-parameter Selection}

Frequently, the hyper-parameter $\lambda$, the parameter used to adjust the amount of regularization applied to the solution, is set by tweaking its value over successive runs until an image that resembles the expected image appears. This procedure roughly corresponds to two different procedures for choosing the hyper-parameter value: the Morozov distinguishability criteria and the L-curve method.[41, 42] These methods both strive to set the hyper-parameter in a rigorous and repeatable manner, so no more regularization is applied than is strictly necessary to overcome the noise in the measurements.

The Morozov discrepancy principle asserts that regularization should be limited to a level where the solution fits the data no more tightly than the noise level.

$$
\left\|\mathbf{J} \mathbf{c}_{\lambda}-\mathbf{z}\right\| \leq \varepsilon
$$

Morozov requires prior knowledge or some estimate of the norm of the measurement noise $\varepsilon$ which is not necessarily available. For tank studies, it is possible to characterize the system, but in biomedical scenarios, this is frequently not possible due 
issues such as electrode movement and posture change. With knowledge of the norm of the measurement noise, the appropriate hyper-parameter value can be found by solving for the hyper-parameter $\lambda$ in the regularized solution when the error norm equals the noise norm.

The L-curve, on the other hand, explores the solution space by plotting the norm of the regularized solution $\left\|\mathbf{L} \mathbf{c}_{\lambda}\right\|$ versus the norm of the residual $\left\|\mathbf{J c}_{\lambda}-\mathbf{z}\right\|$. The ideal hyper-parameter, using this plot, is at the "L" in the curve where the norm of the regularized solution falls drastically with respect to the norm of the residual and represents the point at which the regularization is suppressing the noise without hiding image features. (Figure 4.2)

The L-curve method relies on a reasonably smooth plot of the norms, but for EIT, this plot can be irregular resulting in error-prone results. [43] The L-curve method also requires many solutions to form the plot of the norms which can be time consuming. A similar technique is Generalized Cross-Validation (GCV) which uses error curves to estimate when an appropriate level of regularization is being applied.

For the purposes of this thesis, the hyper-parameter is set through trial-and-error; though, this is not the most rigorous possible technique. 


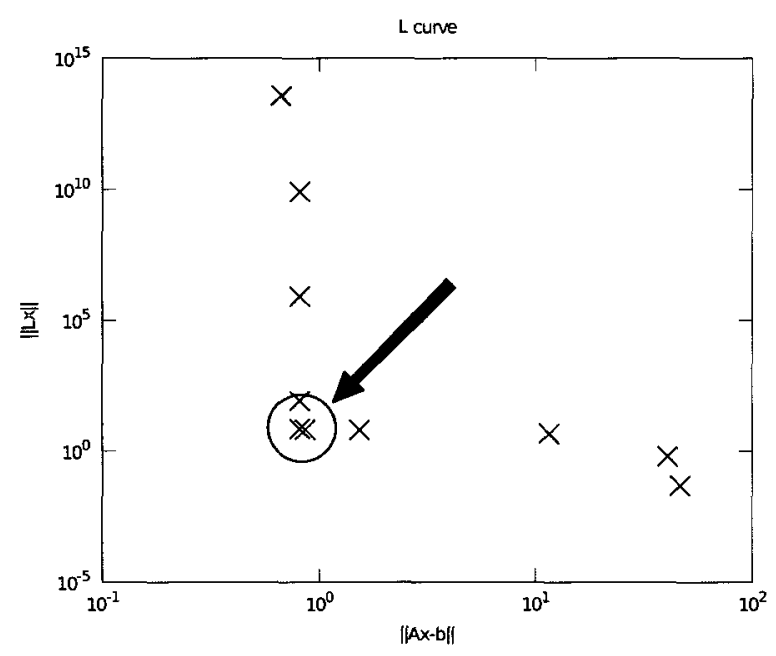

Figure 4.2: Hyper-parameter selection with the L-curve: back-projection algorithm on CT scan simulation with partial data; for the simulated noise level, the hyperparameter corresponding to the reconstructions at the corner of the curve where || $\mathbf{L} \mathbf{c}_{\lambda} \| \simeq 1$ and $\left\|\mathbf{J} \mathbf{c}_{\lambda}-\mathbf{z}\right\| \simeq 1$ is the optimal one for this scenario 


\section{Chapter 5}

\section{Boundary Movement}

Boundary shape uncertainty has been a problem since the inception of Electrical Impedance Tomography (EIT). Electrode positioning errors have been recognized as one of the major factors preventing in vivo, absolute conductivity reconstructions.[44] In biomedical EIT, imaging of the chest immediately encounters these problems due to chest expansion in subjects during breathing and posture change.[45, 46, 47] Theoretically, it has been shown that, given an isotropic conductivity and complete boundary data, a three-dimensional reconstruction of both electrode position and conductivity is possible.[48] The lungs represent an isotropic conductivity if the regions near the boundary containing muscle and other anisotropic material are ignored.

Various electrode positioning algorithms have been implemented previously including $[49,50]$. This thesis focuses on [51] where electrode position is directly manipulated.

In practical EIT of objects with fixed boundaries, it would be unusual to use 
the electrical measurement to recover the boundary shape, as one could employ mechanical or optical measurement devices to determine the external shape of the body and the position of the electrodes. $[52,53]$ In the case of the chest, however, the boundary shape changes with breathing, so it is desirable to correct the boundary shape using the EIT data so that a consistent isotropic conductivity can be fitted to the data. This should result in a distorted image due to the anisotropic nature of chest muscle yet still preserve useful features of the lungs.

\subsection{An Electrode Movement Algorithm}

This section summarizes material from [51] and [54].

This formulation takes the conductivity changes and electrode movement from difference EIT data and forms a regularized inverse with an augmented Jacobian. The augmented Jacobian takes into account the regular conductivity change and an additional factor: electrode movement. Rephrasing the inverse problem with this new electrode movement variable $r$ gives

$$
\mathbf{z}=\mathbf{T}\left[\mathbf{x}\left(\sigma_{2}, r_{2}\right)-\mathbf{x}\left(\sigma_{1}, r_{1}\right)\right]
$$

where $\mathbf{T}$ selects the electrode voltages from the full Finite Element Method (FEM) mesh voltages and $\mathbf{z}$ is the change in measured voltages on the boundary of the domain.

The additional factor in this algorithm is the variable position of electrodes. They must be accounted for in the inverse problem. To do this, the Maximum A Posteriori (MAP) estimate $\mathbf{c}_{\mathrm{MAP}}$ is used where the Jacobian $\mathbf{J}$ and regularization 
covariance matrix inverse $\mathbf{R}$ are expanded to add a section that represents movement along the coordinate axis for each electrode. (Figure 5.1) The MAP estimate of conductivity is

$$
\mathbf{c}_{\mathrm{MAP}}=\arg \min _{\mathrm{MAP}}\|\mathbf{J} \mathbf{c}-\mathbf{z}\|_{\sum_{n}^{-1}}^{2}+\left\|\mathbf{c}-\mathbf{c}_{0}\right\|_{\sum_{x}^{-1}}^{2}
$$

which closely resembles the generalized Tikhonov formulation. The regularization covariance matrix $\sum_{x}^{-1}=\mathbf{R}$ can be seen to be the smoothing norm in generalized Tikhonov regularization where $\mathbf{R}=\mathbf{L}^{\top} \mathbf{L}$. The measurement covariance matrix $\sum_{n}^{-1}$ corresponds to the generalized Tikhonov measurement noise estimate $\mathbf{W}$.

The columns $k$ of the Jacobian $\mathbf{J}$ can be approximated by repeated calculations of the forward model as each of the FEM's electrode positions and mesh element conductivities $k$ is individually perturbed by a small amount.

$$
\mathbf{J}_{k} \simeq \mathbf{T}\left[\frac{\mathbf{x}\left(\sigma_{0}+\Delta \sigma_{k}\right)-\mathbf{x}\left(\sigma_{0}\right)}{\Delta \sigma_{k}}\right]
$$

The regularization covariance matrix has an additional block on the diagonal added to enforce a smoothing criteria on adjacent electrodes with a small positive cost function to penalize solutions that translate all electrodes. Also, the regularization covariance matrix assumes no correlation between conductivity and electrode movement, though this is not strictly true, rather than assign an incorrect correlation that may bias the image and introduce unexpected artifacts.

The inverse problem, when formulated using the Tikhonov generalized form, has a hyper-parameter $\lambda$ to control how much regularization is applied to the solution for conductivity. Similarly, the regularization of the electrode movement is controlled via a new hyper-parameter $\mu$. 


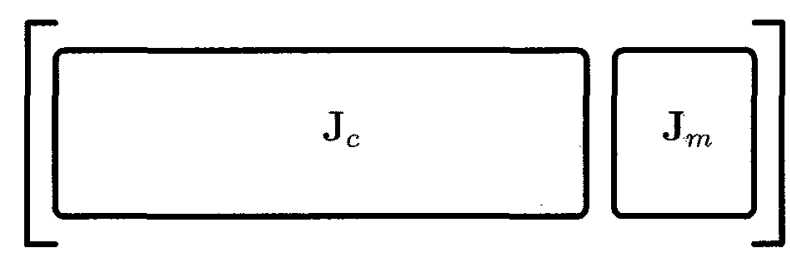

(a) Jacobian $\mathbf{J}$

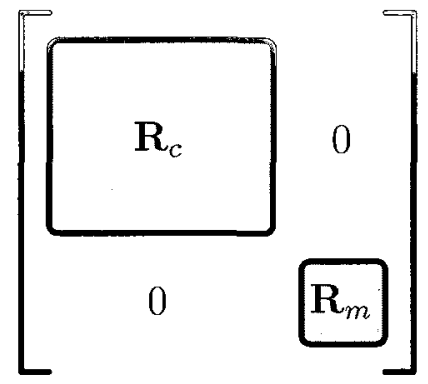

(b) Covariance Matrix $\mathbf{R}$

Figure 5.1: Jacobian and regularization matrices for electrode movement; the conductivity Jacobian $\mathbf{J}_{c}$ has a row per measurement and a column per element (or node if first-order conductivity discretization is used) of the FEM mesh, the movement Jacobian $\mathbf{J}_{m}$ has two (or three in three dimensions) columns per electrode one for each coordinate axis, the covariance matrices are square where the conductivity $\mathbf{R}_{c}$ has a row/column per element/node and movement $\mathbf{R}_{m}$ has two (or three) rows/columns per electrode

Soleimani, et al. [51] implemented a discrete Laplacian for the conductivity regularization; though, there are many choices available. A discrete Laplacian was also selected for the electrode movement regularization because it was thought reasonable to require that the boundary deformation be "smooth". The regularization matrix in the generalized Tikhonov formulation (4.16) takes the form

$$
\mathbf{L}^{\top} \mathbf{L}= \begin{cases}n_{D}+1 & \text { if } i=j \text { and } i \leq n_{N} \\ -1 & \text { if element } i \text { is adjacent to } j \text { and } i \leq n_{N} \\ 2.1 \mu^{2} & \text { if } i=j \text { and } i>n_{N} \\ -\mu^{2} & \text { if electrode } i \text { is adjacent to } j \text { and } i>n_{N} \\ 0 & \text { otherwise }\end{cases}
$$

where $n_{D}$ is the number of dimensions: two for two-dimensional and three for three- 
dimensional reconstructions. For the indices of the regularization matrix, $n_{N}$ indicates the delineation between the conductivity regularization and the electrode movement regularization.

The movement hyper-parameter $\mu$ is the relative weighting in the regularization matrix between conductivity change and electrode movement. The conductivity changes for breathing are on the order of the initial conductivity estimate $\sigma_{0}$, and chest expansion has been found to be approximately $5 \%$ of medium diameter. This gives an initial estimate for the movement hyper-parameter of $\mu=1 / 0.05=20$.

For the purposes of this thesis, the movement hyper-parameter $\mu$ is set through trial-and-error; though, this is not the most rigorous possible technique.

The Jacobian calculation (5.3) can be made more efficient by using a rank-one matrix perturbation as shown in [54]. The electrode movement rank-one perturbation optimization has been observed to reduce the calculation time required for image reconstructions that account for electrode movement by two to three orders of magnitude. The scheme creates a separable product of the system matrix in the forward problem $\mathbf{A}_{s}$ into a connectivity matrix $\mathbf{C}$, an unconnected shape matrix $\mathbf{S}$, and conductivity matrix $\mathbf{D}$.

$$
\mathbf{A}_{s}=\mathbf{C}^{\top} \mathbf{A}(\mathbf{r}, \sigma)_{e} \mathbf{C} \quad \rightarrow \quad \mathbf{A}_{s}=\mathbf{C}^{\top} \mathbf{S}(\mathbf{r}) \mathbf{D}(\sigma) \mathbf{C}
$$

To determine the conductivity related portion of the Jacobian, the partial derivative of the node voltages with respect to conductivity is manipulated as follows:

$$
\frac{\partial \mathbf{x}_{0}}{\partial \sigma}=\frac{\partial\left(\mathbf{A}_{0}^{-1} \mathbf{b}\right)}{\partial \sigma}=\mathbf{A}_{0}^{-1} \frac{\partial \mathbf{A}_{0}}{\partial \sigma} \mathbf{A}_{0}^{-1} \mathbf{b}=\mathbf{A}_{0}^{-1} \frac{\partial \mathbf{A}_{0}}{\partial \sigma} \mathbf{x}_{0}
$$


where

$$
\frac{\partial \mathbb{A}_{0}}{\partial \sigma}=\mathbb{C}^{\top} \mathbb{S} \frac{\partial \mathbb{D}}{\partial \sigma} \mathbb{C}
$$

and $\partial \mathbf{D} / \partial \sigma$ is approximated using the regular conductivity perturbation technique. To determine the electrode movement related components of the Jacobian, an analogous manipulation of the equations is performed as follows:

$$
\frac{\partial \mathbf{x}_{0}}{\partial \mathbf{r}}=\frac{\partial\left(\mathbf{A}_{0}^{-1} \mathbf{b}\right)}{\partial \mathbf{r}}=\mathbf{A}_{0}^{-1} \frac{\partial \mathbf{A}_{0}}{\partial \mathbf{r}} \mathbf{A}_{0}^{-1} \mathbf{b}=\mathbf{A}_{0}^{-1} \frac{\partial \mathbf{A}_{0}}{\partial \mathbf{r}} \mathbf{x}_{0}
$$

where

$$
\frac{\partial \mathbf{A}_{0}}{\partial \mathbf{r}}=\mathbf{C}^{\top} \frac{\partial \mathbf{S}}{\partial \mathbf{r}} \mathbf{D C}
$$

For first-order linear interpolatory expansion functions in two dimensions, the FEM expansion functions are defined with a block matrix $\mathbf{S}_{e}$ for each element of the mesh, and the blocks are arranged on the diagonal into the unconnected shape matrix $\mathbf{S}$. The element shape matrix $\mathbf{S}_{e}$ is

$$
\mathbf{S}_{e}=\frac{2}{n_{D} !} \frac{1}{\operatorname{det}(\mathbf{M})} \mathbf{N}^{\top} \mathbf{N}
$$

where the number of dimensions $n_{D}$ is two and the matrix $\mathbf{M}$, in two dimensions, is defined as

$$
\mathbf{M}=\left[\begin{array}{ccc}
1 & r_{x, 1} & r_{y, 1} \\
1 & r_{x, 2} & r_{y, 2} \\
1 & r_{x, 3} & r_{y, 3}
\end{array}\right]^{-1}
$$

The coordinates of the triangular element vertices are $\left(r_{x, n}, r_{y, n}\right)$ for the $n$-th vertex. The matrix $\mathbf{N}$ is the matrix $\mathbf{M}$ but removing the first row. 
The rank-one perturbation of the electrode positions requires the derivative of the Sherman-Morrison formula for a small perturbation $\alpha$

$$
\begin{aligned}
\left(\mathbf{X}+\alpha \mathbf{u} \mathbf{v}^{\top}\right)^{-1} & =\mathbf{X}^{-1}-\frac{\alpha \mathbf{X}^{-1} \mathbf{u} \mathbf{v}^{\top} \mathbf{X}^{-1}}{1+\alpha \mathbf{v}^{\top} \mathbf{X}^{-1} \mathbf{u}} \quad, \quad \alpha \neq-\left(\mathbf{v}^{\top} \mathbf{X}^{-1} \mathbf{u}\right)^{-1} \\
\frac{\partial}{\partial \alpha}\left(\mathbf{X}+\alpha \mathbf{u} \mathbf{v}^{\top}\right)^{-1} & =-\frac{\mathbf{X}^{-1} \mathbf{u} \mathbf{v}^{\top} \mathbf{X}^{-1}}{1+\alpha \mathbf{v}^{\top} \mathbf{X}^{-1} \mathbf{u}} \stackrel{\alpha \rightarrow 0}{\simeq} \quad-\mathbf{X}^{-1} \mathbf{u} \mathbf{v}^{\top} \mathbf{X}^{-1}
\end{aligned}
$$

The rank-one perturbation also uses the derivative of the matrix determinant lemma for a small perturbation

$$
\begin{aligned}
\operatorname{det}\left(\mathbf{X}+\alpha \mathbf{u} \mathbf{v}^{\top}\right) & =1+\alpha \mathbf{v}^{\top} \mathbf{X}^{-1} \mathbf{u} \operatorname{det}(\mathbf{X}) \\
\frac{\partial}{\partial \alpha} \operatorname{det}\left(\mathbf{X}+\alpha \mathbf{u} \mathbf{v}^{\top}\right) & =\mathbf{v}^{\top} \mathbf{X}^{-1} \mathbf{u} \operatorname{det}(\mathbf{X})
\end{aligned}
$$

Knowing that the FEM's structural equations (5.10) are first-order, the partial derivative with respect to electrode movement can be expanded such that

$$
\frac{\partial \mathbf{S}_{e}}{\partial \mathbf{r}}=\frac{2}{n_{D} !}\left(\frac{\partial}{\partial \mathbf{r}}\left(\frac{1}{\operatorname{det}(\mathbf{M})}\right) \mathbf{N}^{\top} \mathbf{N}+\frac{1}{\operatorname{det}(\mathbf{M})} \frac{\partial \mathbf{N}^{\top}}{\partial \mathbf{r}} \mathbf{N}+\frac{1}{\operatorname{det}(\mathbf{M})} \mathbf{N}^{\top} \frac{\partial \mathbf{N}}{\partial \mathbf{r}}\right)
$$

where there are now three partial derivative terms to solve.

The first term involves the inverse absolute value of a determinant. The sign function $\operatorname{sgn}(x)$ is used to resolve the absolute value of $x$ and gives +1 if greater than $0,-1$ if less than 0 , and 0 if $x=0$. Using the property of inverse determinants $\operatorname{det}\left(\mathbf{X}^{-1}\right)=1 / \operatorname{det}(\mathbf{X})$, the perturbation approximation $\mathbf{X}^{-1} \simeq\left(\mathbf{X}+\alpha \mathbf{u v}^{\top}\right)^{-1}$ for small perturbations $\alpha \rightarrow 0$, and the derivative of the matrix determinant lemma 
(5.15) provides the following solution:

$$
\begin{aligned}
\frac{\partial}{\partial \mathbf{r}} \frac{1}{\operatorname{det}(\mathbf{M}) \mid} & =\frac{\partial}{\partial \mathbf{r}} \frac{-\operatorname{sgn}(\operatorname{det}(\mathbf{M}))}{\operatorname{det}(\mathbf{M})}=-\operatorname{sgn}(\operatorname{det}(\mathbf{M})) \frac{\partial}{\partial \mathbf{r}} \operatorname{det}\left(\mathbf{M}^{-1}\right) \\
& =-\operatorname{sgn}(\operatorname{det}(\mathbf{M})) \mathbf{v}^{\top} \mathbf{M} \mathbf{u} \operatorname{det}\left(\mathbf{M}^{-1}\right) \\
& =\frac{\mathbf{v}^{\top} \mathbf{M} \mathbf{u}}{|\operatorname{det}(\mathbf{M})|}
\end{aligned}
$$

The second derivative term is a transpose of the third derivative term.

$$
\frac{\partial \mathbf{N}^{\top}}{\partial \mathbf{r}}=\left(\frac{\partial \mathbf{N}}{\partial \mathbf{r}}\right)^{\top}
$$

The third derivative term is manipulated using the matrix $\mathbf{N}$, which is the matrix $\mathbf{M}$ with the first row removed $\mathbf{N}=\left.\mathbf{M}\right|_{\text {/row1 }}$, the perturbation approximation $\mathbf{X}^{-1} \simeq$ $\left(\mathbf{X}+\alpha \mathbf{u v}^{\top}\right)^{-1}$ for small perturbations $\alpha \rightarrow 0$, and the derivative of the ShermanMorrison formula (5.13) to obtain the following:

$$
\begin{aligned}
\frac{\partial \mathbf{N}}{\partial \mathbf{r}} & =\left.\left.\frac{\partial \mathbf{M}}{\partial \mathbf{r}}\right|_{/ \text {row } 1} \stackrel{\mathbf{M}=\mathbf{P}^{-1}}{=} \frac{\partial}{\partial \alpha}\left(\mathbf{P}+\alpha \mathbf{u v}^{\top}\right)^{-1}\right|_{/ \text {row } 1} \\
& \simeq-\left.\left(\mathbf{P}^{-1} \mathbf{u v}^{\top} \mathbf{P}^{-1}\right)\right|_{/ \text {row1 }} \\
& =-\left.\left(\mathbf{M u v}^{\top} \mathbf{M}\right)\right|_{/ \text {row } 1}
\end{aligned}
$$

The vectors $\mathbf{u}$ and $\mathbf{v}$ are applied to select the direction of perturbation for each electrode node by selecting the node in the matrix $\mathbf{M}^{-1}$ which is to be perturbed. The vector $\mathbf{u}$ selects the row, and the vector $\mathbf{v}$ selects the column when each vector has a single 1 and is zero in all other entries. Thus, a perturbation of an electrode that exists at local node 1 , along the $y$-axis, would require the vectors $\mathbf{u}=\left[\begin{array}{lll}1 & 0 & 0\end{array}\right]^{\top}$ and $\mathbf{v}=\left[\begin{array}{lll}0 & 0 & 1\end{array}\right]^{\top}$. 
This boundary movement algorithm and the rank-one matrix perturbation optimization are implemented in the Electrical Impedance and Diffuse Optics Reconstruction Software (EIDORS) package and used extensively throughout this work.

\subsection{Conformal Motion}

As shown later in this thesis, conformal motions are relevant to the boundary movement problem. Conformal motions are angle preserving. They maintain the angular relationship between intersecting curves while not constraining changes in the length of those curves.

Adding or multiplying conformal motions results in a new conformal motion that is a linear combination of the two. This allows the construction of complicated deformations in two dimensions from four basic types of conformal motion: translation, rotation, dilation, and inversion. With the first three, translation, rotation, and dilation, it is straight forward to visualize how an infinitesimally small triangle will have the lengths of its sides change while the angles of the corners remain fixed. Inversions are more challenging to visualize, but in the extreme, they are a means of turning our triangle "inside-out." Combining a translation and inversion gives a conformal motion that may be familiar to engineers: the Joukowski transformation. This deformation distorts a circle into an airfoil-like shape. It allows the application of closed form analytic solutions for potential flow around a circular object to be applied to a "Joukowski airfoil".[55] (Figure 5.2)

The inversions provide the most interesting set of conformal deformations for EIT since EIT is invariant under translation and rotation. Centered dilations provide a 
simple, symmetric conformal deformation. Combinations of conformal deformations can result in deformations that are not intuitively conformal in their appearance. (Figure 5.3)

The focus of this thesis is upon two-dimensional conformality, but there are threedimensional conformal deformations. The set of conformal deformations in three dimensions is significantly restricted when compared to the two-dimensional case but it is not clear whether similarly complex effects can occur in three-dimensional reconstructions.

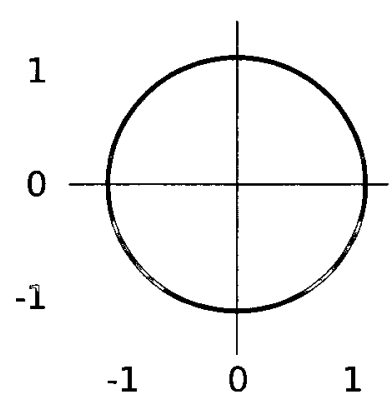

(a) circle

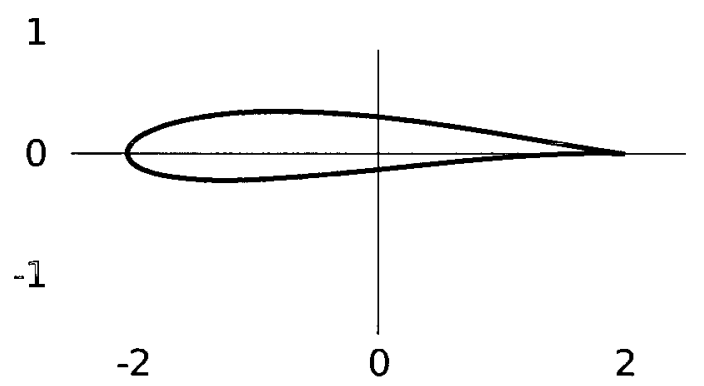

(b) airfoil

Figure 5.2: The Joukowski transform is a well known conformal mapping; a circle (a) with radius 1.121 and center $(-0.117,0.048)$ is transformed into an idealized airfoil (b) via the Joukowski transform $z \mapsto z+1 / z$

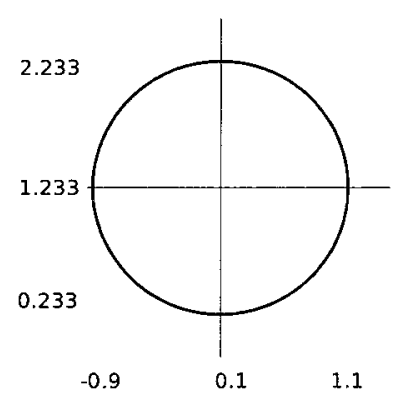

(a) circle

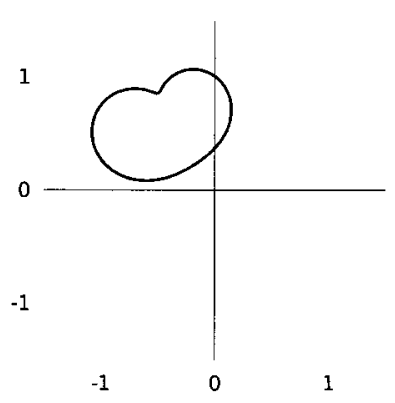

(b) transformation

Figure 5.3: A less intuitive conformal motion; a circle (a) with radius 1.121 and center $(0.1,1.233)$ is transformed into new shape $(b)$ via the conformal transform $z \mapsto 1 /(z+1)^{2 / 3}(z-1)^{2 / 3}$ 


\section{Part II}

\section{Contributions}




\section{Chapter 6}

\section{Phantom}

The material in this chapter was presented at the $9^{\text {th }}$ International Conference on Biomedical Applications of Electrical Impedance Tomography, Dartmouth, USA (EIT 2008). [8]

In biomedical Electrical Impedance Tomography (EIT), it has long been suspected that errors in the knowledge of the boundary shape are an important factor in the accuracy of reconstruction. This effect is most important in chest EIT where the chest shape deforms as the patient breathes and changes posture[46][56].

An algorithm has previously been developed that assumes, given an isotropic conductivity distribution, that a boundary movement will result in an anisotropic distribution. $[48,51]$ The result is that a deformation of the boundary can be detected by a change in the boundary measurements. This additional information has been used to implement an algorithm that perturbs electrode positions in an attempt to better fit the measurement data for small changes in the boundary.

The following describes a deformable phantom, used to obtain in vitro EIT mea- 
surements and evaluate the performance of the electrode perturbation algorithm on arbitrary boundary deformations obtained using this phantom.

\subsection{Construction}

The phantom is constructed of a sponge rubber plumbing gasket placed in a shallow pan. The gasket forms a thick rubber ring that is easily compressed yet rigid enough to return to its original shape easily.

Sixteen electrodes were constructed from stainless-steel wire pressed into the gasket and then looped over the edge of the gasket such that they lay along the inner wall of the gasket in a vertical orientation. An additional stainless-steel electrode placed roughly in the geometric center of the gasket formed the ground connection. A shallow layer of saline solution was employed to limit conductivity in the vertical direction, thereby presenting an approximately two-dimensional section in the experimental measurements. The electrodes were each wired to a terminal that was bolted to the plastic pan which provided a good connection to the EIT system. (Figure 6.1)

The thickness of the gasket allowed the electrodes to be securely attached to the phantom as it is deformed. The thickness of the gasket also provided electrical insulation between the saline solution inside and outside the gasket.

\subsection{Method}

A 16 electrode Goe-MF II EIT system (Viasys Healthcare, Höchberg, Germany) was used for taking measurements from the deformable phantom. The phantom was submerged in a saline bath $(0.68 \% \mathrm{NaCl}$ salinity) such that the bottom of the 


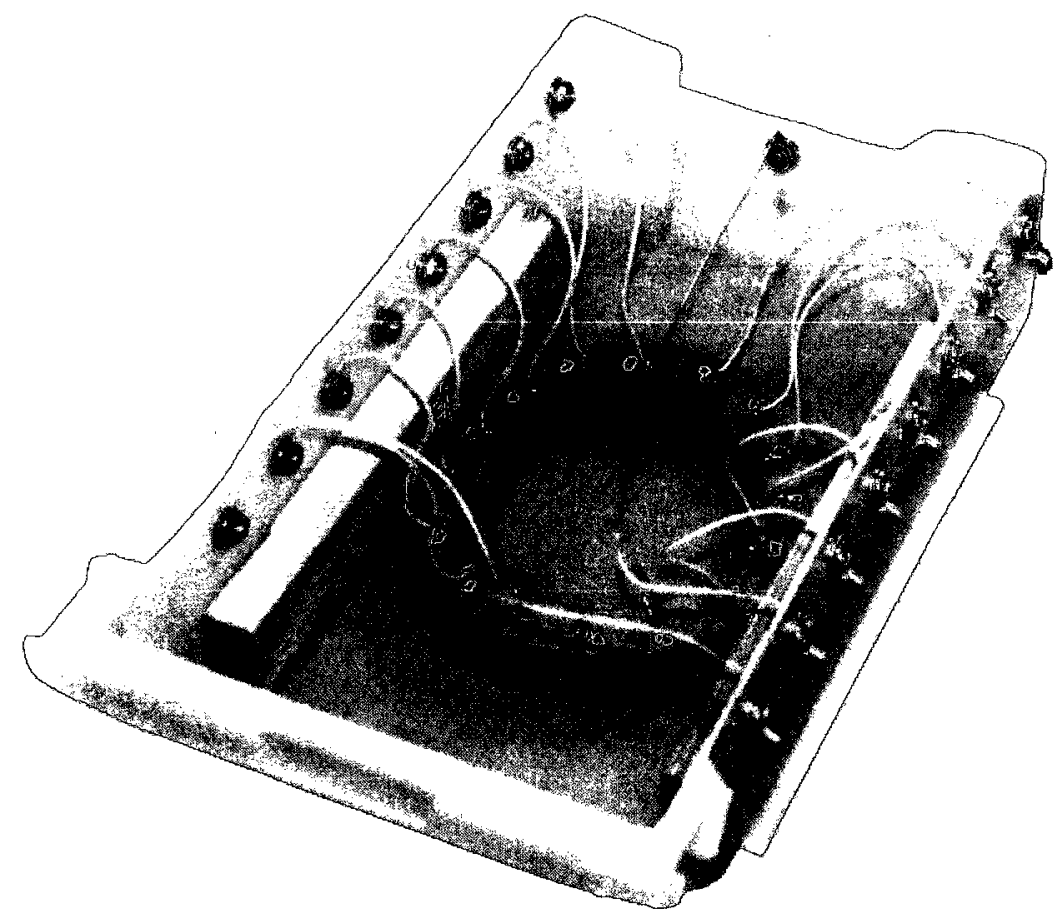

Figure 6.1: Photograph of the phantom, built from a rubber gasket and stainless steel electrodes. The yellow dish is filled with a saline solution. 
ring was in contact with the bottom of the container, and the top of the ring broke the surface which provides insulation between the inside and outside of the ring. Salinity was set such that a nominal electrical impedance of $250 \Omega$ was measured between adjacent electrodes.

\subsection{Deformations}

Measurements were taken with the phantom in

- an approximately circular (relaxed) arrangement,

- with a side-to-side compression from two points, and

- with the ring under three points of compression. (Figure 6.2)

For each of these deformations, measurements were obtained with

- a conductive target,

- a non-conductive target, and

- no target.

An iron cylinder with a diameter of $6 \mathrm{~mm}$ was used as the conductive target, and a glass cylinder of $42 \mathrm{~mm}$ was used for the non-conductive target.

\subsection{Electrode Displacements}

The true physical displacements of the electrodes were found by taking a digital photograph from above the phantom. A piece of graph paper was placed under 


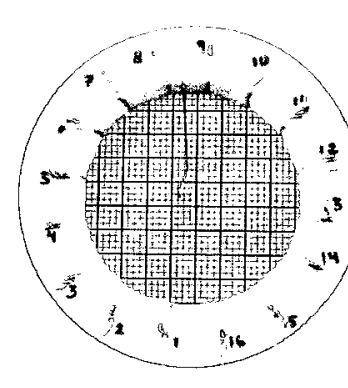

(a)

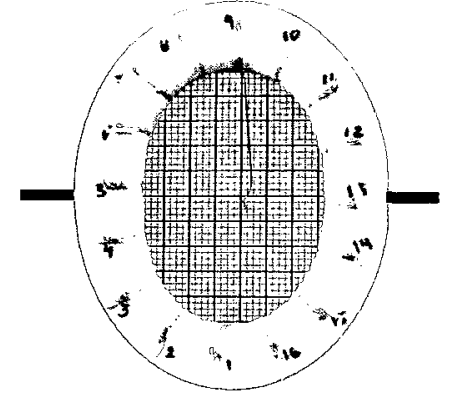

(b)

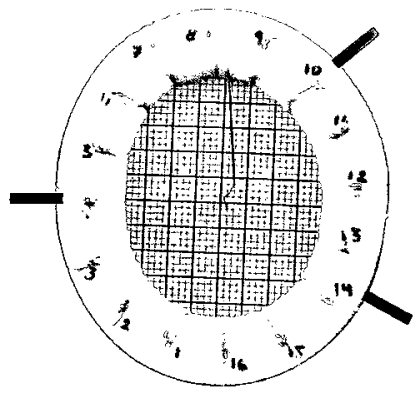

(c)

Figure 6.2: Compressions: none (a), 2 points (b), 3 points (c)

the phantom and was taped to the bottom of the pan. The locations of the electrodes were measured from the photograph, in pixels, and then normalized using the graph paper grid. This corrected scaling issues due to different camera altitudes above the phantom as well as skew issues caused by the lens axis not being exactly perpendicular to the phantom's floor.

The norm of the error in initial electrode location relative to a circular model of a radius matching the mean radius of the true electrode locations was found to be 0.0662 . (The norm, in this case, being the square root of the sum of squared errors.) This indicated a fairly good match between the initial state of the phantom and the circular model in the reconstruction. The majority of the error norm was found to be related to errors in the angular location of the electrodes, rather than the radial displacement. The mean of angular electrode error was found to be $1.3^{\circ}$, where electrodes were expected every $22.5^{\circ}$ : a $5.8 \%$ mean angular error.

The estimated electrode displacements were found using the electrode perturbation algorithm as described earlier. Boundaries were fitted to both the estimated and true electrode displacements using a radial Fourier series which allowed both 
rotation and the Fourier series radial displacements:

$$
\begin{aligned}
& \theta^{\prime}=\theta+\theta_{k} \\
& r^{\prime}=r \sum_{n=0}^{N} a_{n} \cos \left(n \theta^{\prime}\right)+b_{n} \sin \left(n \theta^{\prime}\right)
\end{aligned}
$$

For the initial terms of the summation $n=0$, note that $\cos \left(n \theta^{\prime}\right)=1$, and therefore, $a_{0}$ forms a radial scaling term while $b_{0}$ disappears $\operatorname{since} \sin \left(n \theta^{\prime}\right)=0$. With $N=5$, reasonable fits to the displaced electrode locations were obtained.

Figures $6.3,6.4,6.5$ and 6.6 are examples of the fitting of boundaries to the true and estimated electrode locations and comparison of the resulting boundary parameters. In each case subfigure (a) shows the true electrode displacements (green arrows) as measured from a digital photo with the fitted boundary (blue dashed line). The boundary was fitted using the radial Fourier series (6.1a) (6.1b). Subfigure (b) shows a naïve reconstruction without the electrode perturbation algorithm. Note in particular the significant artifacts around the boundary in the reconstruction in all cases. Subfigure (c) shows the reconstructed electrode movement (green arrows) with fitted boundary (blue dashed line). Subfigure (d) shows the a bar chart of the difference in parameter values between the fitted boundary of the true and reconstructed images. The "parameters" on the y-axis correspond to $1: \theta_{k}, 2: a_{0}, 3,4: a_{1}, b_{1}, 5,6: a_{2}, b_{2}, 7,8$ : $a_{3}, b_{3}, 9,10: a_{4}, b_{4}$ from (6.1a) (6.1b).

Figures $6.3,6.5$ and 6.6 are examples of the boundary fitting working well. The electrode movement algorithm significantly reduced the artifacts in the reconstructed image. The conductive target did not show up very well when compared to the insulating target which may be due to the small size of the conductive target. The most 
significant difference in the fitted boundaries is the scaling parameter $a_{0}$. Figure 6.5 shows the boundary fitting algorithm failing to fit the estimated electrode movement.

Using the fitted boundary, the norm of the error in the parameters was found. (Figure 6.3) For most cases except where no deformation occurred, it was found that the majority of the error was in the $a_{0}$ term which corresponds to a simple dilation. (Table 6.1)

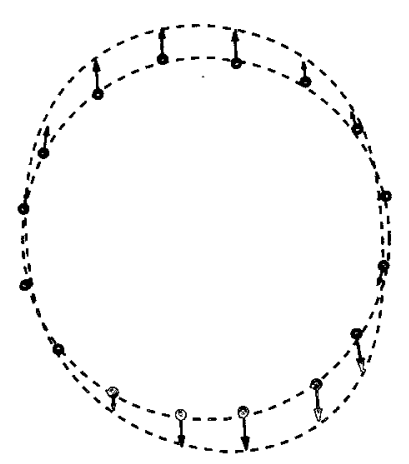

(a) true displacements

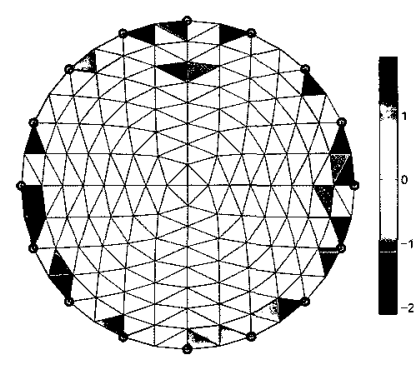

(b) naïve reconstruction

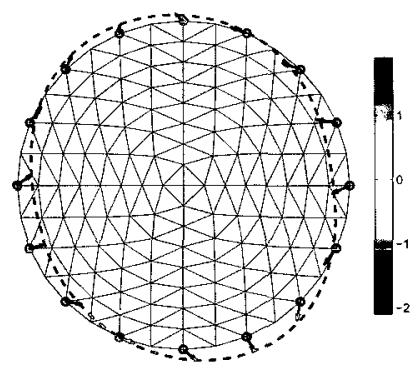

(c) movement reconstruction

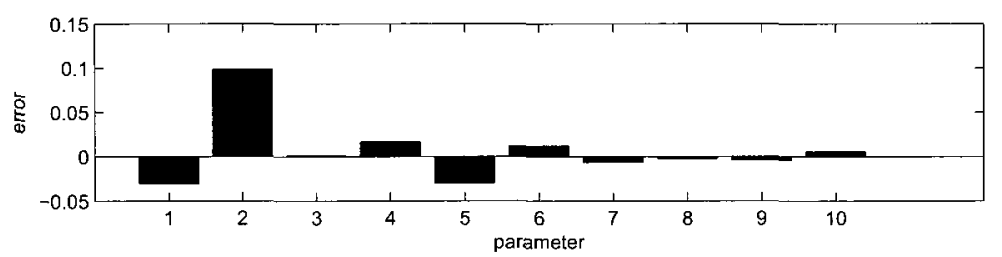

(d) parameter error

Figure 6.3: Boundary reconstruction from electrode movement for 3-point compression with a conductive target showing artifact reduction when accounting for electrode movement and reasonable agreement between fitted boundaries.

\subsection{Summary and Discussion}

This work describes the construction of a deformable EIT phantom applicable for testing two-dimensional deformations. Results with experimental data suggest that 
Table 6.1: Boundary Reconstruction Accuracy

\begin{tabular}{rlccc}
\hline & & \multicolumn{2}{c}{ Parameter Error Norm } & \\
\cline { 3 - 4 } Deformation & Target & Full & Without $a_{0}{ }^{*}$ & Comment \\
\hline \multirow{2}{*}{ None } & none & 0.004 & 0.004 & \\
& insulator & 0.930 & 0.930 & \\
& conductor & 0.235 & 0.235 & \\
& none & 0.276 & 0.239 & \\
& insulator & 0.561 & 0.530 & Figure 6.4 \\
& conductor & 0.139 & 0.121 & Figure 6.5 \\
& none & 0.185 & 0.049 & \\
& insulator & 0.087 & 0.086 & Figure 6.6 \\
& conductor & 0.109 & 0.048 & Figure 6.3 \\
\hline
\end{tabular}

${ }^{*} a_{0}$ parameter from the Fourier series (6.1b)

some electrode movement and boundary distortions can be reconstructed based on conductivity changes alone while reducing image artifacts in the process. The electrode displacement errors were evaluated against photos of the true electrode positions. The error calculations show that the electrode perturbation algorithm does not perfectly reconstruct the electrode movement. A significant class of deformations that is not accurately captured by this algorithm includes those that result in dilation of the boundary.

With a number of the experiments, notably the two-point compressions, there are visible errors in the "true" deformation where the phantom's total circumference should not be changing significantly. These errors are due to the coordinate normalization where the graph paper grid beneath the phantom was measured and used to correct for the varying altitudes above the phantom from which the true measurement photographs were taken. A single grid unit of $2.5 \mathrm{~mm}$ square was used as the basis for normalizing coordinates. In retrospect, choosing a larger basis measure 
would have reduced the error margin in the normalization. An alternative would be to fix the camera's location with an armature such that there would be a common unit length when measuring coordinates from the photographs.

The quality of results for the Fourier fitting algorithm are dependent on the electrode movements. Movements that result in angular changes in electrode locations are not well accounted for by the fitting algorithm since it only allows for a single universal rotation. Potentially, an algorithm that allowed more freedom in the angular direction could result in closer fits. Despite these shortcomings, the boundary fits were generally found to be reasonable.

The data handling and reconstruction algorithms (both naïve and electrode movement) were those implemented in the Electrical Impedance and Diffuse Optics Reconstruction Software (EIDORS) package, version 3.3. Boundary fitting and associated analysis was implemented as separate code. 


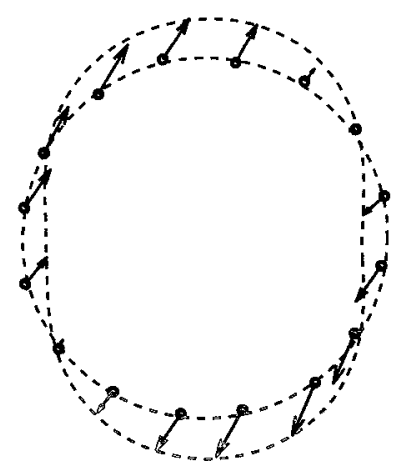

(a) true displacements

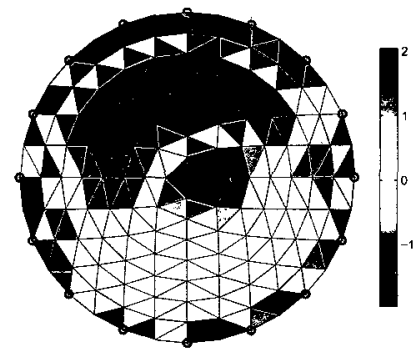

(b) naïve reconstruction

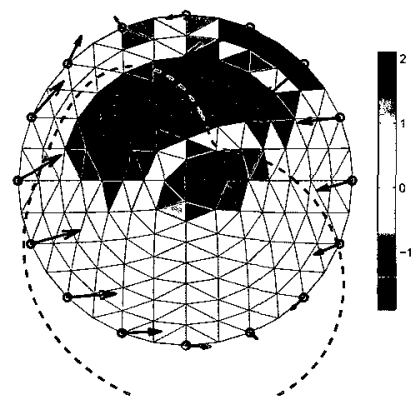

(c) movement reconstruction

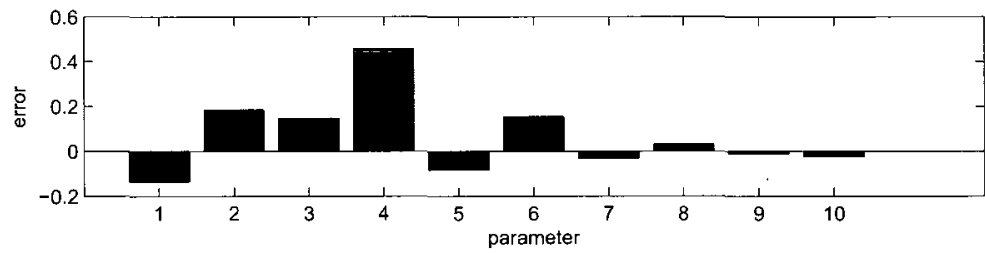

(d) parameter error

Figure 6.4: Boundary reconstruction from electrode movement: 2-point compression with insulating target showing poorly fitted boundary in reconstruction (c); true displacement scaled by 0.6 to correct for photo-scaling errors 


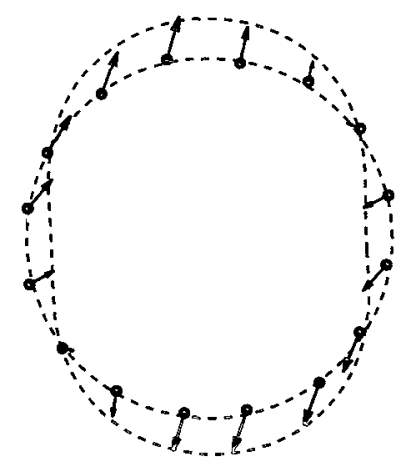

(a) true displacements

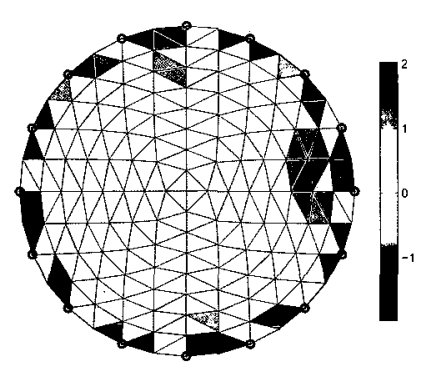

(b) naïve reconstruction

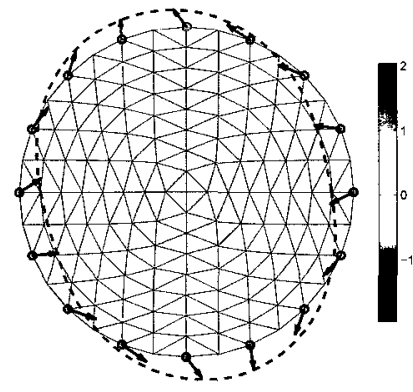

(c) movement reconstruction

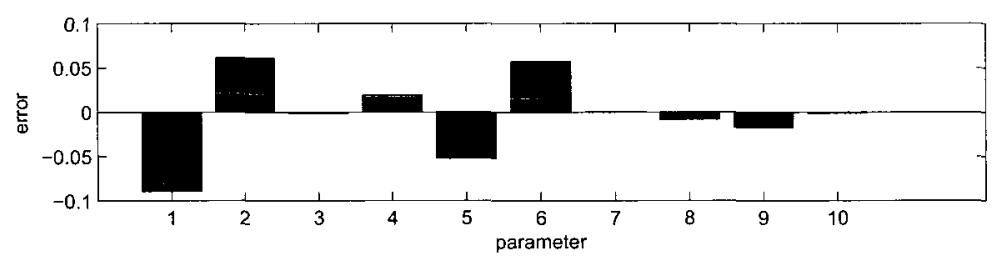

(d) parameter error

Figure 6.5: Boundary reconstruction from electrode movement: 2-point compression with conductive target showing artifact reduction when accounting for electrode movement and reasonable agreement between fitted boundaries; true displacement scaled by 0.7 to correct for photo-scaling errors 


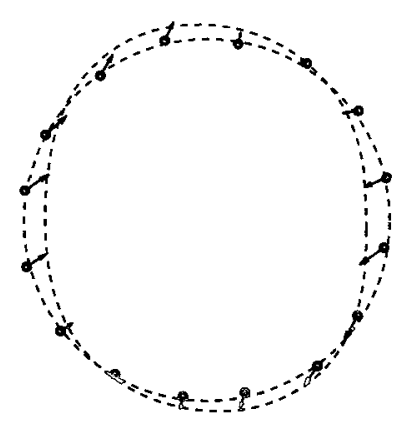

(a) true displacements

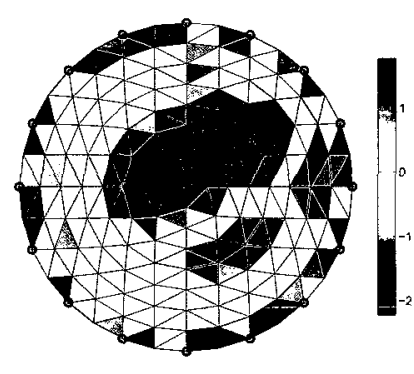

(b) naïve reconstruction

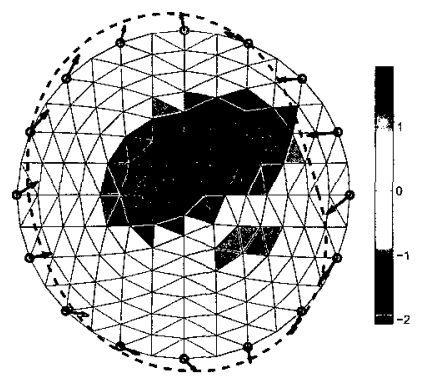

(c) movement reconstruction

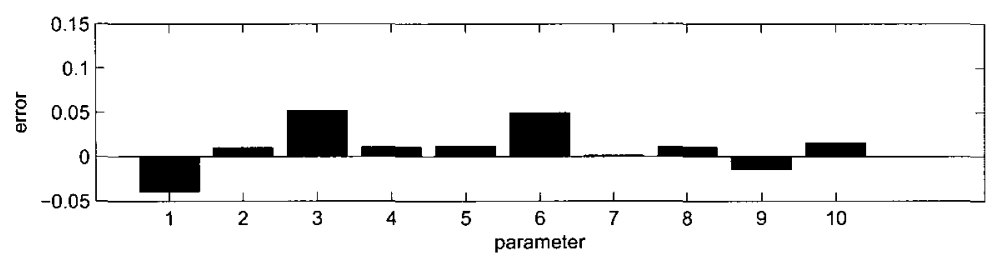

(d) parameter error

Figure 6.6: Boundary reconstruction from electrode movement: 3-point compression with insulating target showing artifact reduction when accounting for electrode movement and reasonable agreement between fitted boundaries; true displacement scaled by 0.8 to correct for photo-scaling errors 


\section{Chapter 7}

\section{Simulated Conformal Motion}

The material in this chapter was also presented at the $9^{\text {th }}$ International Conference on Biomedical Applications of Electrical Impedance Tomography, Dartmouth, USA (EIT 2008).[8]

The ability and limits of Electrical Impedance Tomography (EIT) to resolve conductivity changes and reject boundary distortion are explored below. It is shown that the theoretical results given in [48] still hold in the case of a finite number of electrodes and a finite element discretization of the forward problem. Our example simulations in two dimensions, using a linearization of the forward problem, suggest that the boundary shape and electrode positions can be recovered up to an infinitesimal conformal map. This provides an adequate and necessary correction for acceptable reconstruction of the conductivity. 


\subsection{Simulated Results}

In order to explore the effect of reconstructing EIT images from media with conformal and non-conformal conductivity changes, two-dimensional simulations using the Electrical Impedance and Diffuse Optics Reconstruction Software (EIDORS) package were constructed.[10] Using a 576 element Finite Element Method (FEM) mesh which filled the unit circle and using adjacent stimulation and measurement on 16 point electrodes, two distortion fields were simulated. Representing each boundary point by a complex $z=x+\mathrm{i} y$, the distortions were as follows:

$$
\begin{array}{lr}
z \rightarrow 0.99 x+\mathrm{i} 1.01 y & \text { Non-conformal } \\
z \rightarrow z+0.01 z^{2} & \text { Conformal }
\end{array}
$$

Additionally, one small conductive and one small non-conductive target were simulated.

Using the approach of Soleimani et al [51], the time-difference conductivity and movement images were reconstructed. An assumed movement to conductivity change parameter $\mu=0.01$ was used. For comparison, an algorithm was used which assumes no boundary movement.

Results are shown in Figure 7.1 illustrating that conformal distortion fields cannot be distinguished from conductivity changes. When examining Figure 7.1 row-by-row, the top subfigures (a), (b), (c) show a Non-conformal Distortion field $z \rightarrow 0.99 x+$ i1.01y. The middle subfigures $(d),(e),(f)$ show a Conformal Distortion field $z \rightarrow$ $z+0.01 z^{2}$. The bottom subfigures $(g),(h),(i)$ show a combined Conformal and Nonconformal Distortion field. When examining Figure 7.1 column-by-column, the left- 
most subfigures $(a),(d),(g)$ show the simulation movement and conductivity changes (the forward model). The middle subfigures (b), (e), (h) show the reconstruction assuming only conductivity change. The right-most subfigures $(c),(f),(i)$ show the reconstruction of conductivity change and movement. The green arrows indicate electrode movement (40× exaggeration).

For the case of non-conformal movements, there are dramatic artifacts in the conductivity only reconstruction, and there is a clear benefit to movement reconstruction. In the case of conformal movements, no such benefit is seen, and the movement reconstruction is unable to detect movement. The combined distortion shows reduced artifacts courtesy of the electrode movement reconstruction algorithm but shows significant errors in the reconstructed electrode movement.

Following the same layout as Figure 7.1, the phantom deformations of Chapter 6 where simulated by applying the deformation as indicated by the fitted boundary parameters. The true boundary deformation (bottom row) was fitted from photographs of the deformation, while the boundary resulting from the phantom reconstruction (top row) were assumed to be similar to the non-conformal reconstruction of Figure 7.1. Finally, the difference between the two boundary parameters was used to perform a final set of simulations (middle row). For all simulations in Figure 7.2 and Figure 7.3, the interior conductivity changes were the same as those in Figure 7.2. (Figure 7.2 and 7.3) Results were inconclusive and appear to be compromised by the inability of the boundary fitting algorithm to match angular movement of the electrodes. 


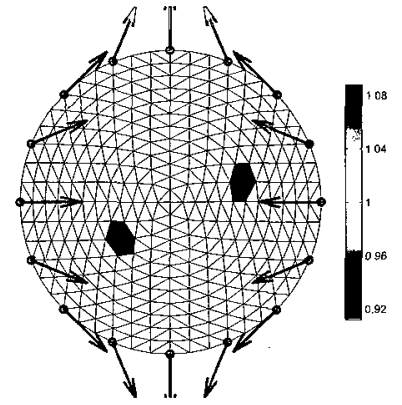

(a)

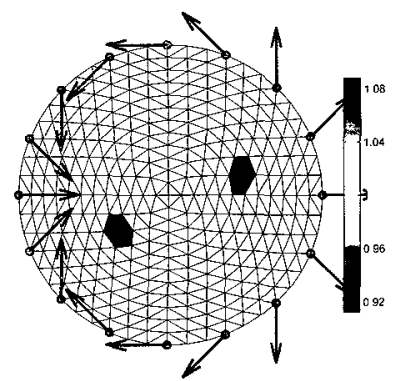

(d)

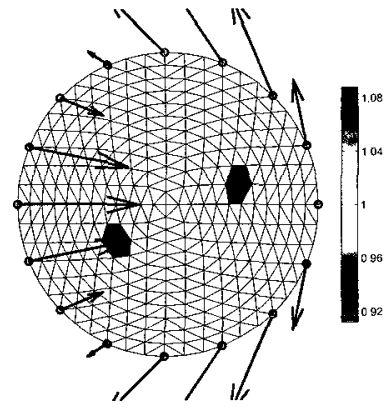

(g)

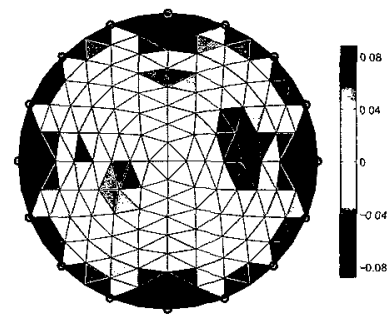

(b)

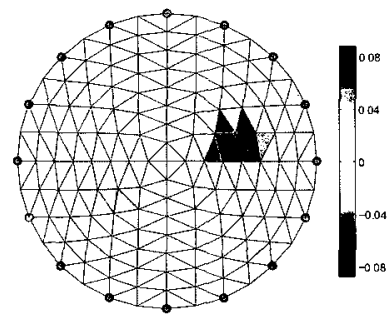

(e)

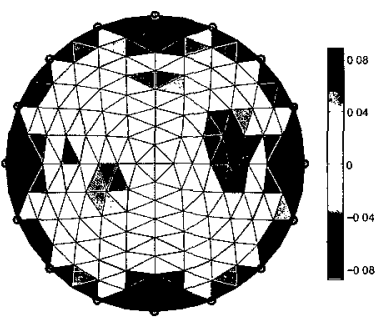

(h)

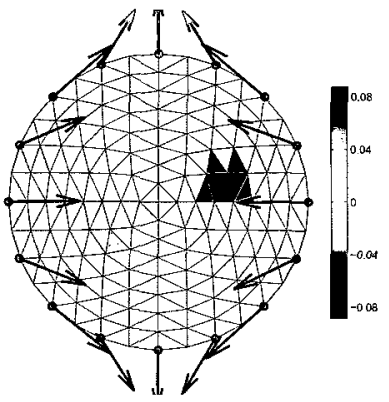

(c)

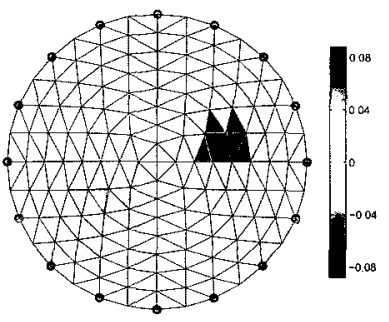

(f)

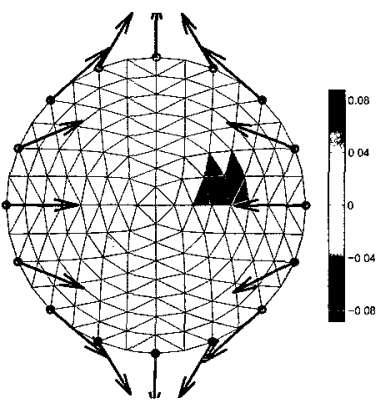

(i)

Figure 7.1: Images reconstructed from three different movement patterns illustrating that conformal distortion fields cannot be distinguished from conductivity changes. 


\subsection{Summary and Discussion}

This work describes conformal and non-conformal vector fields applied to electrode movement and boundary distortion in EIT. Results in simulation suggest that, with non-conformal mappings, electrode movement and boundary distortions can be reconstructed based on conductivity changes alone while reducing image artifacts in the process, whereas conformal mappings remain undetected but do not introduce artifacts.

The results of the phantom shape deformation parameter simulations do not match the conformal and non-conformal simulations. This appears to be a result of the boundary parameter fitting algorithm not allowing angular movement of the electrodes except when the change is uniform amongst electrodes. Further work to improve the boundary fitting algorithm could significantly improve the quality of these results.

The forward models and reconstruction algorithms (both naïve and electrode movement) were those implemented in the EIDORS package, version 3.3. The shape deformations were implemented as separate code. 


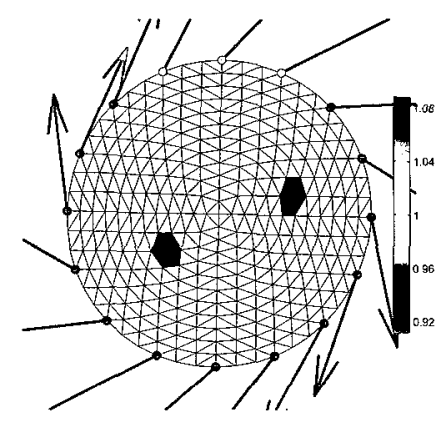

(a)

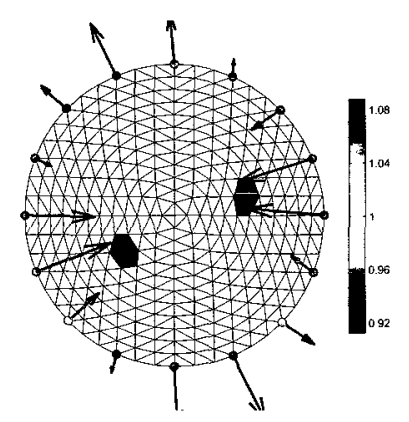

(d)

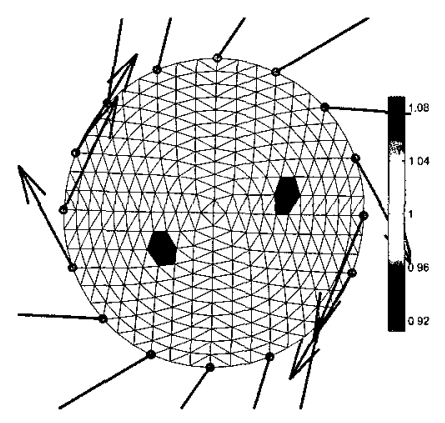

(g)

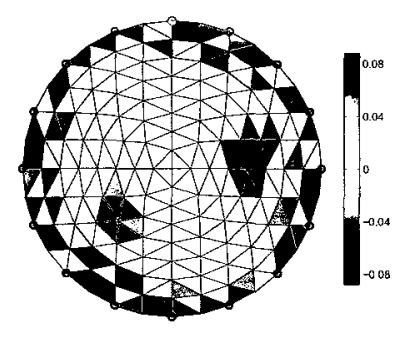

(b)

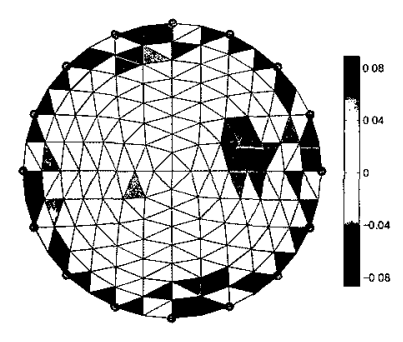

(e)

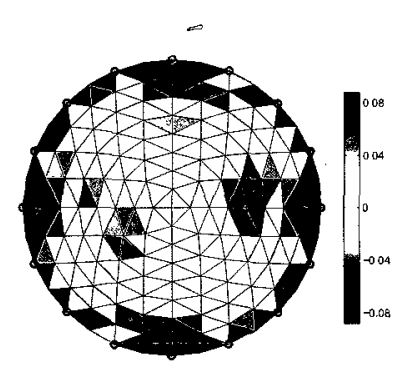

(h)

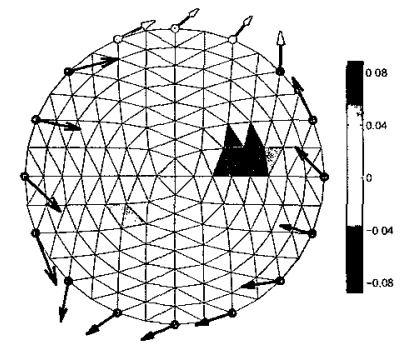

(c)

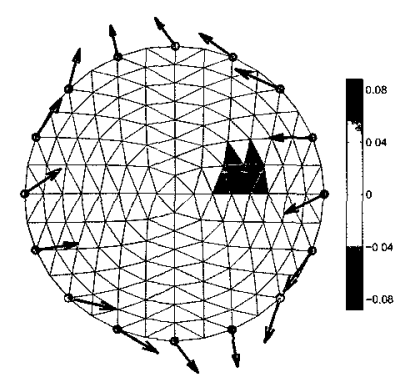

(f)

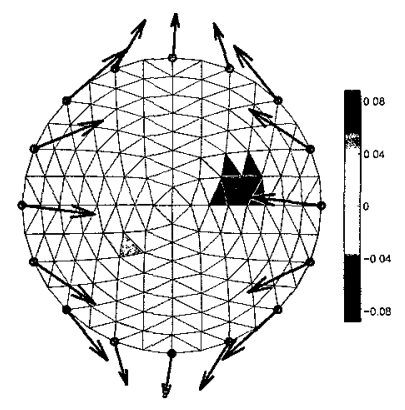

(i)

Figure 7.2: Simulation of two point deformation; images reconstructed from portions of the approximated phantom deformation. No clear conclusions regarding conformal movement can be drawn when comparing these reconstructions. 


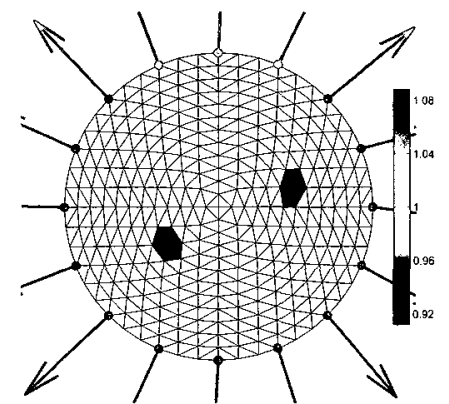

(a)

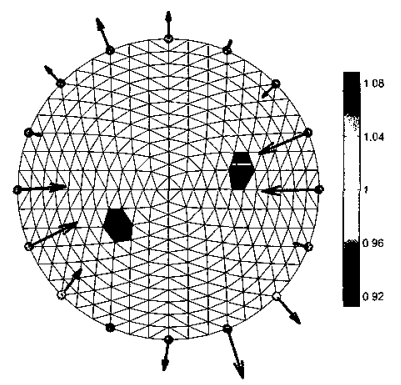

(d)

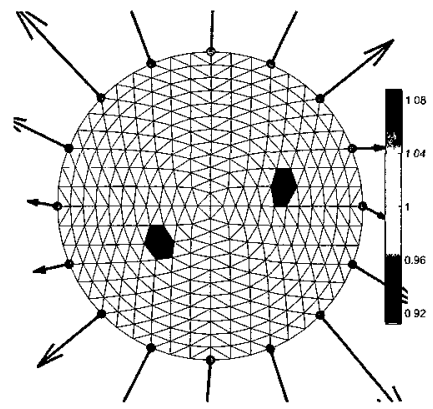

(g)

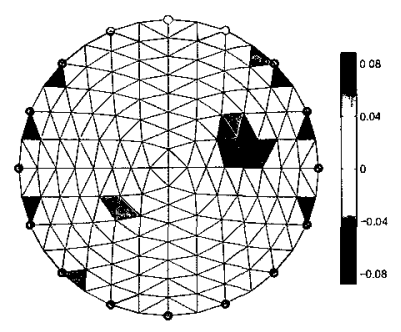

(b)

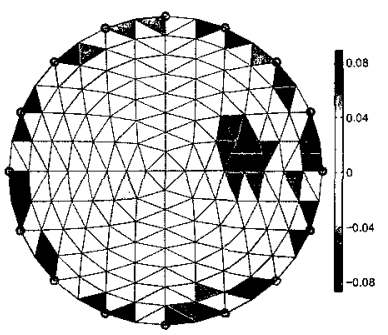

(e)

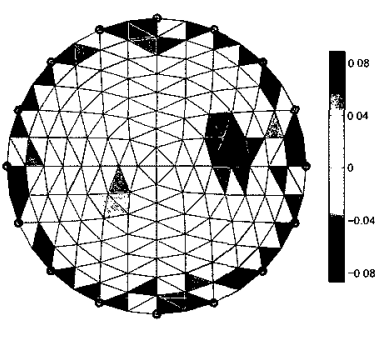

(h)

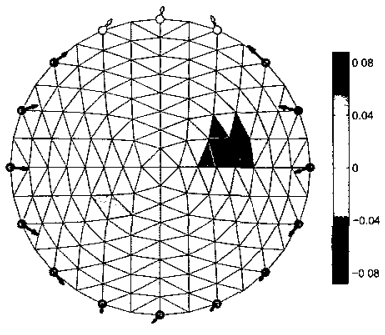

(c)

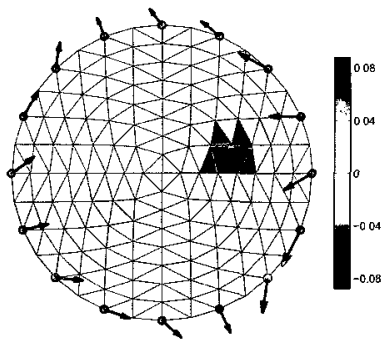

(f)

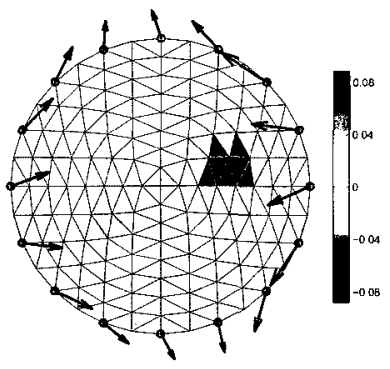

(i)

Figure 7.3: Simulation of three point deformation; images reconstructed from portions of the approximated phantom deformation. No clear conclusions regarding conformal movement can be drawn when comparing these reconstructions. 


\section{Chapter 8}

\section{Finite Element Deformations}

The material in this chapter is based on a conference paper presented at the $10^{\text {th }}$ International Conference on Biomedical Applications of Electrical Impedance Tomography, Manchester, UK (EIT 2009).[9]

In the previous chapter, two-dimensional shape deformations where explored through simulations. This exploration is extended into the theoretical realm by exploring what occurs to the interior conductivity when a boundary deformation occurs in Electrical Impedance Tomography (EIT).

\subsection{Conductivity as a Tensor}

When a boundary deformation occurs, how does the new conductivity have to change to allow the voltages over the domain to remain the same? In this case, conductivity $\sigma$ is treated as a tensor; it is allowed to be either isotropic or anisotropic.

To explore this question, the weak form of EIT's constitutive equation is examined 
as it is applied in the EIT Finite Element Method (FEM) model:

$$
\int_{\Omega} u \nabla \cdot \sigma \nabla \phi d V=0
$$

and when a deformation occurs, it results in a new conductivity $\sigma^{\prime}$ and potential distribution over the domain $\phi^{\prime}$ as well as some change in the volume $d V^{\prime}$ over which the integral is calculated. A new test function $u^{\prime}$ is also introduced.

$$
\int u \nabla \cdot \sigma \nabla \phi d V=\int u^{\prime} \nabla \cdot \sigma^{\prime} \nabla \phi^{\prime} d V^{\prime}
$$

In two dimensions, the deformation is defined by some transformation from $(x, y)$ coordinates to $\left(x^{\prime}, y^{\prime}\right)$ coordinates:

$$
\left(x^{\prime}, y^{\prime}\right)=f(x, y)
$$

This can be thought of as a transformation of the coordinate system from a euclidean $x$ - and $y$-axis to $\left(x^{\prime}, y^{\prime}\right)$ in a new coordinate system. For notational consistency, the initial $x$-axis is referred to as $x_{1}$ and the $y$-axis as $x_{2}$, and the new $x^{\prime}$ - and $y^{\prime}$ axes are $X_{1}$ and $X_{2}$. (Figure 8.1) The transformation can be represented by a set of partial derivatives of the transformation function

$$
\left[\begin{array}{c}
\frac{\partial}{\partial x_{1}} \\
\frac{\partial}{\partial x_{2}}
\end{array}\right]=\left[\begin{array}{ll}
a & b \\
c & d
\end{array}\right]\left[\begin{array}{c}
\frac{\partial}{\partial X_{1}} \\
\frac{\partial}{\partial X_{2}}
\end{array}\right]
$$

where $a=\frac{\partial X_{1}}{\partial x_{1}}, b=\frac{\partial X_{2}}{\partial x_{1}}, c=\frac{\partial X_{1}}{\partial x_{2}}$, and $d=\frac{\partial X_{2}}{\partial x_{2}}$. Taking the inverse of the transfor- 

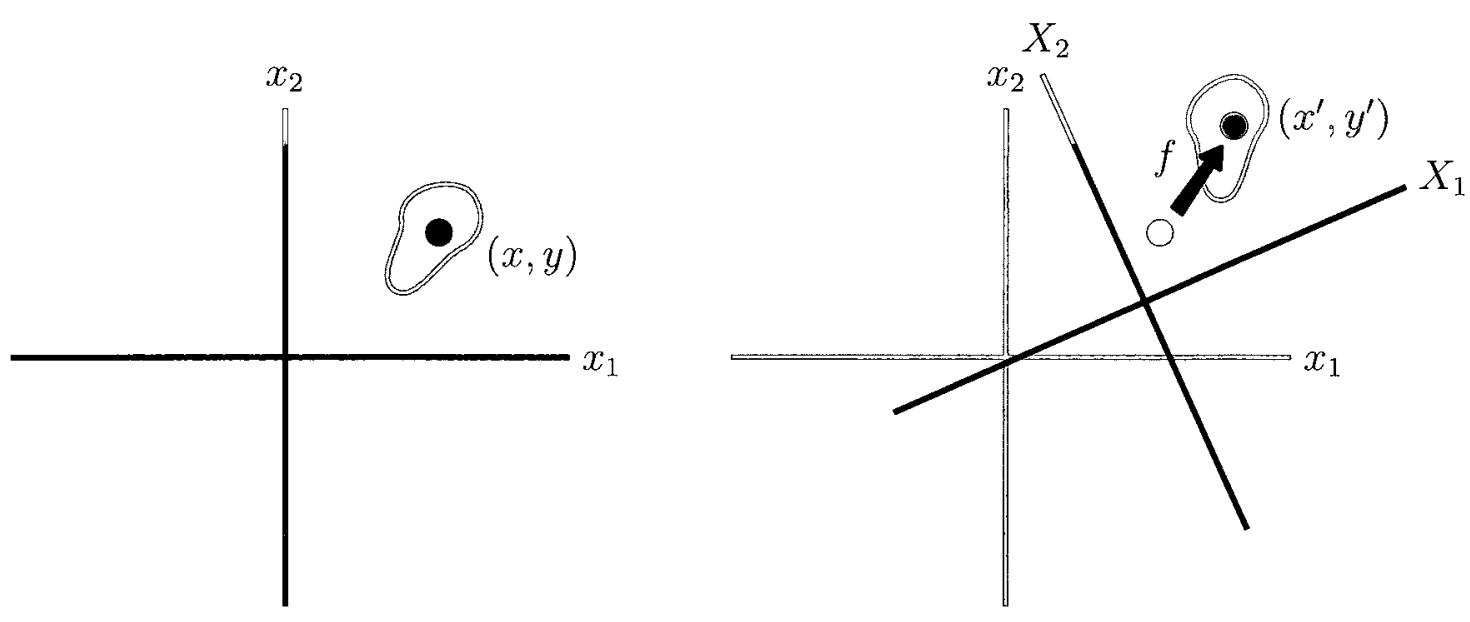

Figure 8.1: A change of coordinate systems transforms the point $(x, y)$ to the new coordinates $\left(x^{\prime}, y^{\prime}\right)$; the old coordinates $(x, y)$ relative to the original $\mathrm{x}$-, $\mathrm{y}$-axis (the $x_{1}$ - and $x_{2}$-axis) are at the same location as the new coordinates relative to the new axis (the $X_{1}$ - and $X_{2}$-axis) where in this case, a translation and rotation have been applied.

mation gives

$$
\left[\begin{array}{c}
\frac{\partial}{\partial X_{1}} \\
\frac{\partial}{\partial X_{2}}
\end{array}\right]=\underbrace{\frac{1}{a d-b c}\left[\begin{array}{cc}
d & -b \\
-c & a
\end{array}\right]}_{\mathbf{T}}\left[\begin{array}{c}
\frac{\partial}{\partial x_{1}} \\
\frac{\partial}{\partial x_{2}}
\end{array}\right]
$$

where $\mathbf{T}$ is the two-by-two matrix inverse (Appendix A.7) of the transformation matrix in (8.4). The volume derivative scales by the transformation where that scaling factor is given by the determinant (Appendix A.1).

$$
d V^{\prime}=\frac{1}{\operatorname{det}(\mathbf{T})} d V
$$

Taking the equivalence equation (8.2), the new conductivity $\sigma^{\prime}$ must be modified in some way if the voltages on the domain are to be equal $\left(\phi=\phi^{\prime}\right)$. The test functions can be chosen arbitrarily and are set equal $\left(u=u^{\prime}\right)$. Expanding (8.2) into 
its matrices gives

$$
\left[\begin{array}{ll}
\frac{\partial}{\partial x_{1}} & \frac{\partial}{\partial x_{2}}
\end{array}\right] \sigma\left[\begin{array}{c}
\frac{\partial}{\partial x_{1}} \\
\frac{\partial}{\partial x_{2}}
\end{array}\right] \phi d V=\left[\begin{array}{ll}
\frac{\partial}{\partial X_{1}} & \frac{\partial}{\partial X_{2}}
\end{array}\right] \sigma^{\prime}\left[\begin{array}{c}
\frac{\partial}{\partial X_{2}} \\
\frac{\partial}{\partial X_{2}}
\end{array}\right] \phi^{\prime} d V^{\prime}
$$

where potential is equal $\left(\phi=\phi^{\prime}\right)$ and applying the partial derivative (8.5) and volume (8.6) transformations to (8.7) gives

$$
\left[\begin{array}{ll}
\frac{\partial}{\partial x_{1}} & \frac{\partial}{\partial x_{2}}
\end{array}\right] \sigma\left[\begin{array}{c}
\frac{\partial}{\partial x_{1}} \\
\frac{\partial}{\partial x_{2}}
\end{array}\right] \phi d V=\left[\begin{array}{ll}
\frac{\partial}{\partial x_{1}} & \frac{\partial}{\partial x_{2}}
\end{array}\right] \mathbf{T}^{\top} \sigma^{\prime} \mathbf{T}\left[\begin{array}{c}
\frac{\partial}{\partial x_{1}} \\
\frac{\partial}{\partial x_{2}}
\end{array}\right] \phi \frac{1}{\operatorname{det}(\mathbf{T})} d V
$$

Simplifying (8.8), where the volume derivative $d V$, potential $\phi$ and partial derivatives cancel, leaves

$$
\sigma=\mathbf{T}^{\top} \sigma^{\prime} \mathbf{T} \frac{1}{\operatorname{det}(\mathbf{T})}
$$

and rearranging for the new conductivity $\sigma^{\prime}$, gives

$$
\sigma^{\prime}=\mathbf{T}^{-\mathrm{T}} \sigma \mathbf{T}^{-1} \operatorname{det}(\mathbf{T})
$$

This equation (8.10) shows that when conductivity $\sigma$ is a tensor, for any transformation $\mathbf{T}$, the conductivity can be adjusted in some manner such that the new boundary data will match the old data. However, there is no guarantee that an initially isotropic conductivity will map to a new conductivity that is isotropic. 


\subsection{Current Density}

If the total current entering or leaving a given electrode does not change, the current density must change when a deformation changes the surface area of the domain connected to the electrode. In two dimensions, intuitively, the current density for an electrode $J_{n}$ must scale with the change in the length of the boundary associated with the electrode $1 / \ell$.

This can be seen when taking the current density equation where the new and old current for electrode $n$ must be the same

$$
\begin{aligned}
I_{n}=\int J_{n} d S & =\int J^{\prime}{ }_{n} d S^{\prime} \\
J^{\prime}{ }_{n} & =J_{n} \frac{d S}{d S^{\prime}}
\end{aligned}
$$

which shows that the change in current density is proportional to the inverse of the change in surface area over which the current is being applied.
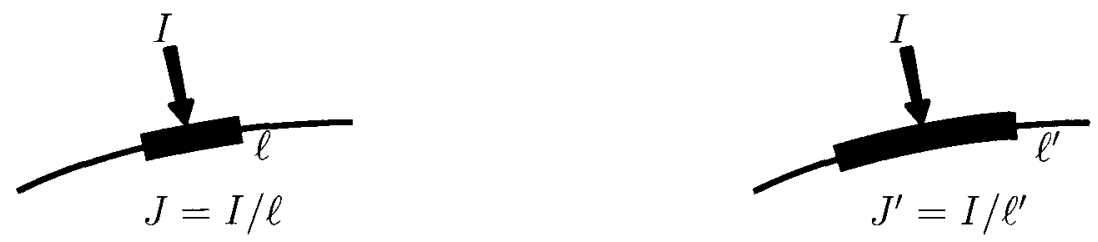

Figure 8.2: Electrode current density under deformation

\subsection{Isotropic Conductivity}

If the measurements $\phi$ remain constant when a deformation occurs and the initial conductivity is isotropic, when is the new conductivity $\sigma^{\prime}$ isotropic? Taking the 
conductivity equivalence (8.10) derived earlier and assuming isotropic conductivities allows the new conductivity $\sigma^{\prime}$ to be pulled out of the middle of the equation such that

$$
\sigma=\sigma^{\prime} \mathbf{T}^{-\mathrm{T}} \mathbf{T}^{-1} \operatorname{det}(\mathbf{T})
$$

where $\mathbf{T}^{-T} \mathbf{T}^{-1} \operatorname{det}(\mathbf{T})$ must be isotropic such that $k \mathbf{I}=\mathbf{T}^{-\top} \mathbf{T}^{-1} \operatorname{det}(\mathbf{T})$.

Expanding and then simplifying the equation gives

$$
\begin{gathered}
k \mathbf{I}=\left[\begin{array}{ll}
a & c \\
b & d
\end{array}\right]\left[\begin{array}{ll}
a & b \\
c & d
\end{array}\right] \frac{1}{a d-b c} \\
k \mathbf{I}=\frac{1}{a d-b c}\left[\begin{array}{ll}
a^{2}+c^{2} & a b+c d \\
a b+c d & b^{2}+d^{2}
\end{array}\right]
\end{gathered}
$$

and solving for $a b+c d=0$ and $a^{2}+c^{2}=b^{2}+d^{2}$ results in $a= \pm d$ and $c=\mp b$. Substituting these into (8.15) gives

$$
\begin{gathered}
k \mathbf{I}=\frac{1}{a^{2}+b^{2}}\left[\begin{array}{cc}
a^{2}+b^{2} & 0 \\
0 & a^{2}+b^{2}
\end{array}\right] \\
k=1
\end{gathered}
$$

The first solution $a=+d$ and $c=-b$ turns out to be the Cauchy-Riemann equations, which indicates that conformal deformations, and only conformal deformations, can result in a new isotropic conductivity if the original conductivity was isotropic. The alternative solution $a=-d$ and $c=+b$ is the first solution multiplied 
by $\left[\begin{array}{cc}1 & 0 \\ 0 & -1\end{array}\right]$, which corresponds to switching from a right-hand rule coordinate system to a left-hand rule coordinate system. (Figure 8.3)

The solution with $k=1$ shows that transformations that map an isotropic conductivity to another isotropic conductivity will have boundary measurements that match. There is no multiplicative constant $k$ which need be applied to the conductivity to achieve identical boundary measurements.

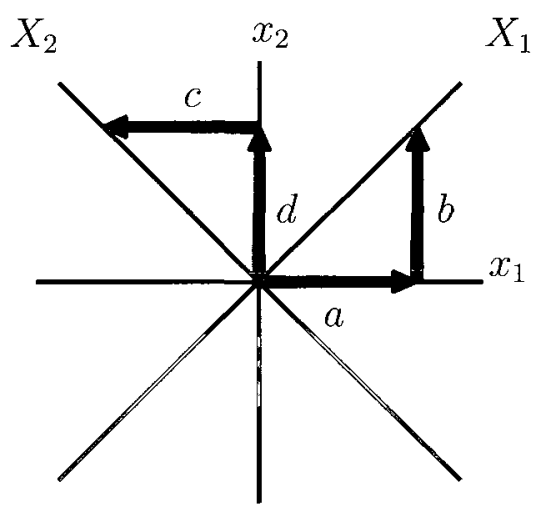

(a) $a=d, b=-c$

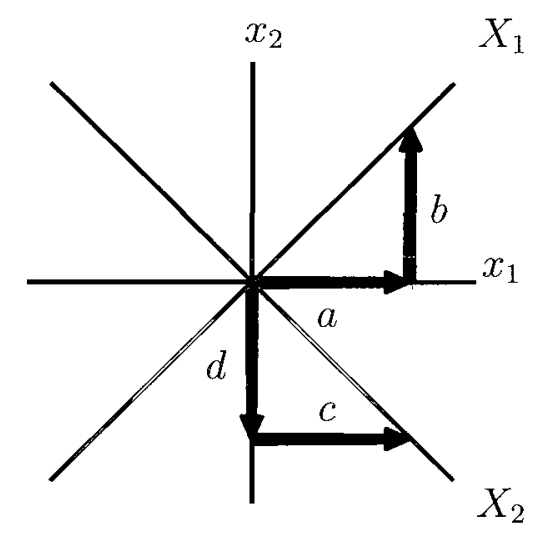

(b) $a=-d, b=c$

Figure 8.3: The two conformal solutions; (a) and (b) are solutions for conformal transformations that will maintain an isotropic conductivity. Note that (b) is a solution that flips the $X_{2}$-axis.

\subsection{Simulation}

To test the behaviour of an isotropic conductivity when a conformal deformation is applied, a convergence study was undertaken. The undeformed simulation consisted of a rectangular domain with an electrode extending across either end. An L-shaped non-conductive region existed in the middle, between the two electrodes (conductiv- 
ity $\sigma=0.01 \mathrm{~S} / \mathrm{m}$, background $1 \mathrm{~S} / \mathrm{m})$. To exactly control the boundary conditions, a Complete Electrode Model (CEM) for each of the two electrodes was approximated using Point Electrode Models (PEMs) at individual nodes and current was distributed across these to achieve the appropriate current density boundary condition. When the deformation was applied, the current density was adjusted by the deformation, and no further manipulation of the PEM currents was required.

At each step, the forward simulation of the conductivity distribution shown in Figure 8.4(a) was computed to find the voltage on the domain at the nodes. The voltage at the FEM nodes was compared to the voltage after the FEM nodes were conformally deformed such that

$$
z \rightarrow \frac{z-20+80 \mathrm{i}}{100}(z+20 i)(z-10 i), \quad z=x+y \mathrm{i}
$$

(shown in Figure 8.4(b)).

The number of nodes in the simulations ranged from 217 nodes to 455 thousand nodes. Throughout this range, the triangular elements remained approximately equilateral. Convergence was measured as the 2-norm of the difference between the original voltages and the voltages on the mesh after the conformal deformation was applied, normalized against the average of the original voltages $\left(\left\|V_{2}-V_{1}\right\| /\left\|V_{1}\right\|\right)$. As the number of nodes increased and consequently, the element size was reduced, convergence of the undeformed and deformed voltages was observed. (Figure 8.4(c)) 


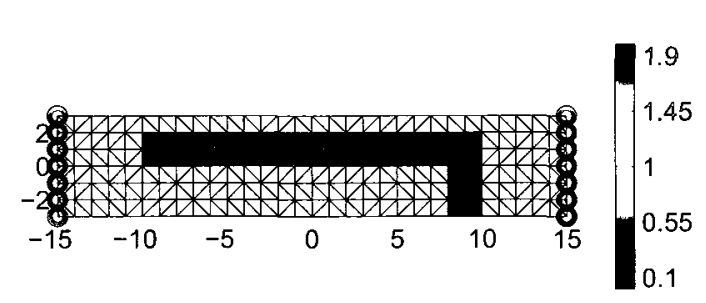

(a) original

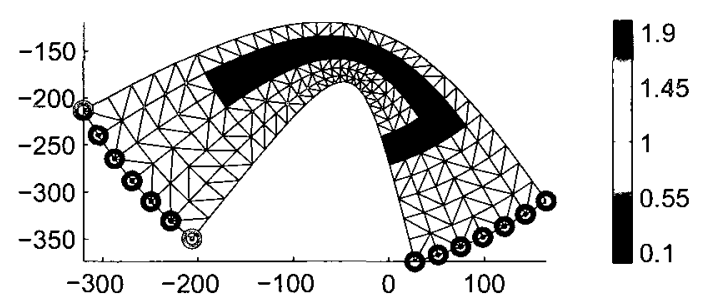

(b) deformed

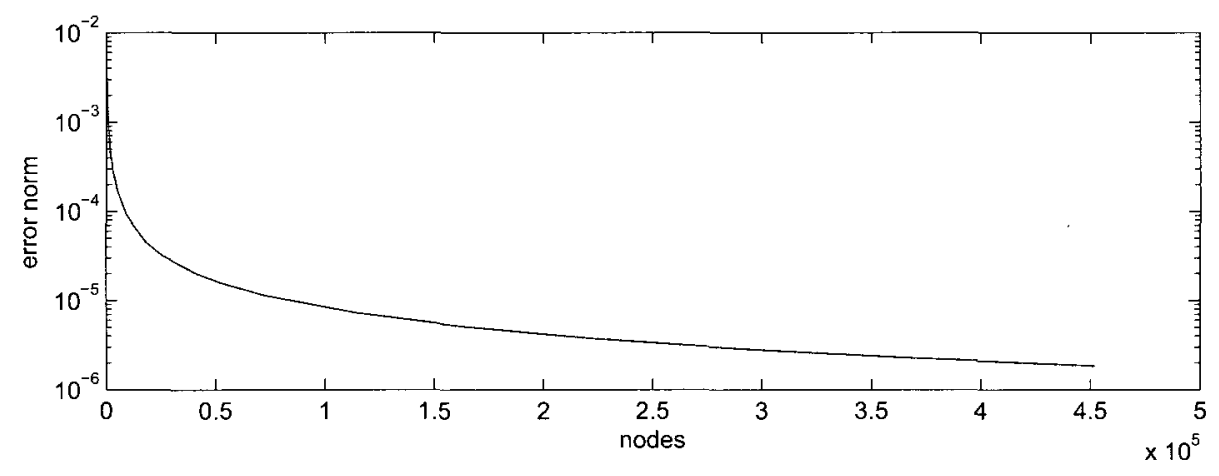

(c) deformed: convergence

Figure 8.4: Conformal deformation convergence; (a) the original problem, (b) the conformal deformation (c) plots of the error norm between the deformed and undeformed voltage distributions; (c) shows solution convergence for (b) as the number of nodes in the FEM mesh increases

\subsection{Summary and Discussion}

This work describes conformal motions in two-dimensional EIT, as applied to the FEM mesh. It is intended to provide a more accessible description of the behaviour of conformal deformations for the engineering community. The results show how conformal motion affects the matrices describing conformal deformations. Simulations show that the conformally deformed mesh converges on the undeformed solution as the mesh size is reduced.

Considering the effect of a conformal motion on a single element of the FEM mesh when using triangular two-dimensional linear expansion functions gives some 
insight into the behaviour of the entire FEM system under conformal motions. The conformal motions, as previously mentioned, can be classified as translation, rotation, dilation, and inversion. Translations and rotations on a single element result in a geometry that is unchanged, and thus, when solving the FEM system as a whole, the values found at transformed nodes of the mesh will be unchanged. Conformal dilations change the geometry of the problem but still result in those same node voltages. When a deformation is applied globally, the nodes in the FEM mesh are displaced, and this can change the angles at the corners of the FEM mesh since the elements themselves are not deformed. They remain strictly triangular. The result is that, for simulations with large elements, the deformed and undeformed solutions do not match. As the element size is reduced, the amount of discrepancy at a local level between an idealized deformation of the element, where its sides are bent, and the actual implementation, where the sides of the elements are always straight, approaches zero. In all conformal deformation cases, the resulting conductivity remains isotropic.

These observations help to explain why the convergence study presented here does not converge more rapidly. The convergence plot clearly shows a trend toward convergence, but when the number of nodes is large, there is still a significant gap between the observed results (an error norm of $10^{-6}$ ) and the hypothetical convergence towards machine precision $\left(10^{-17}\right)$ that might be explained by the strictly triangular nature of the FEM elements.

The convergence studies used the forward solver implemented in the Electrical Impedance and Diffuse Optics Reconstruction Software (EIDORS) package, version 3.3. The shape deformations and analysis were implemented as separate code. 


\section{Chapter 9}

\section{Conformal Motion}

The material in this chapter is based on conference papers presented at the $g^{\text {th }}$ International Conference on Biomedical Applications of Electrical Impedance Tomography, Dartmouth, USA (EIT 2008) and the $10^{\text {th }}$ International Conference on Biomedical Applications of Electrical Impedance Tomography, Manchester, UK (EIT 2009). $[8][9]$

This work describes, in a fundamental mathematical framework, how conformal vector fields do not affect Electrical Impedance Tomography (EIT) images, and exactly which deformations constitute those conformal motions.

In general terms, if a distortion is applied to a domain in two- or three-dimensional space, the assumed isotropy of the conductivity distribution is not preserved.[48] If the conductivity is assumed to be isotropic, generally, the boundary voltage and current data on the distorted domain will not be consistent with an isotropic conductivity. This means that, in the isotropic deformation case, the boundary data contains information about both the conductivity and the boundary shape. This 
additional information has been used to implement an algorithm that perturbs electrode position in an attempt to better fit the measurement data to the reconstruction for small changes in the boundary. [51]

However, not all distortions lead to an anisotropic conductivity containing this additional information. The exception is the distortions that are conformal maps. In two-dimensional space, there are an infinite number of conformal maps, whereas in three dimensions, there is only a finite set of conformal maps: the Möbius transformations.

\subsection{Conformal Maps}

The following develops an understanding of what conformal maps mean in an EIT context and shows that a known boundary deformation can be decomposed into its conformal and non-conformal components.

Let $X$ be a vector field which is assumed to be small in magnitude. A domain $\Omega$ is distorted by the map $x \mapsto x+X \epsilon$. This vector field $X$ indicates the velocity of change in shape over time everywhere over the domain, where multiplying the vector field by some small time $\epsilon$ will give a map that is a new geometry for the domain.(Figure 9.1)

A conformal map is one that preserves the angles but not necessarily the lengths between vectors on the domain. An example is Figure 9.2: a rectangular region with electrodes across the ends and L-shaped inhomogeneity (top left) with a conformal map $z \rightarrow \exp ((z-20-\mathrm{i} 80) / 100) \cdot(z+\mathrm{i} 20) \cdot(z-\mathrm{i} 10)$ applied to it (bottom left). Note that simulated voltages at individual nodes appear to be the same despite the 


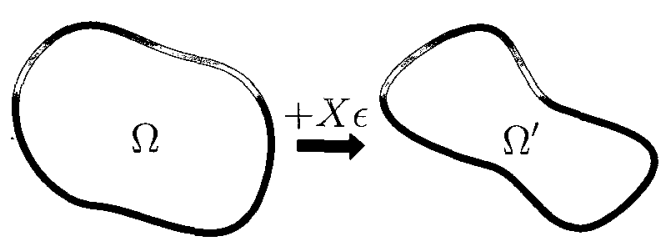

Figure 9.1: A mapping of the domain $\Omega$ to $\Omega^{\prime}$ by the vector field $X$ in some small amount of time $\epsilon$
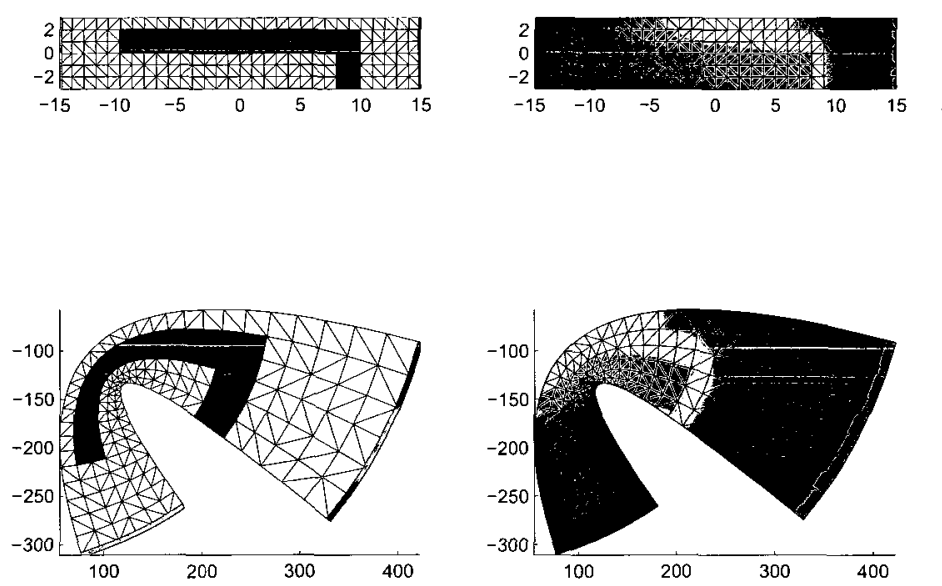

Figure 9.2: A conformal mapping of a rectangular grid illustrating that lines still intersect at $90^{\circ}$ after deformation.

deformation (right-side, top and bottom).

If the map $x \mapsto x+X \epsilon$ is a conformal map, the vector field $X$ is referred to as an infinitesimal conformal map, known classically as an infinitesimal conformal motion, conformal Killing field or more simply a conformal vector field.

A conformal vector field is defined by the fact that, if the distorted domain is to have an isotropic field (e.g. conductivity in EIT) consistent with the boundary conditions, for a change in the boundary of the domain $\Omega$, the vector field $X$ must be conformal and sufficiently smooth. Therefore, $X$ is a conformal vector field if and only if the conformal Killing field equation is satisfied (i.e. the symmetrized 
derivative of $X$ is a multiple of the identity) $(9.1)$. $[57, \S 3.7][58, \S 1.4]$

$$
\frac{\partial X_{i}}{\partial x_{j}}+\frac{\partial X_{j}}{\partial x_{i}}=\alpha \delta_{i j}
$$

where $\alpha$ is a scalar on the domain $\Omega .[57,(3.7 .3)]$

In two dimensions, summing over $i$ and $j$, from taking the trace of (9.1), $\alpha$ must be the divergence of $X$.

$$
\frac{\partial X_{i}}{\partial x_{j}}+\frac{\partial X_{j}}{\partial x_{i}}=(\nabla \cdot X) \delta_{i j}
$$

More explicitly, in two dimensions, (9.1) becomes:

$$
\frac{\partial X_{i}}{\partial x_{j}}+\frac{\partial X_{j}}{\partial x_{i}}=\left(\frac{\partial X_{1}}{\partial x_{1}}+\frac{\partial X_{2}}{\partial x_{2}}\right) \delta_{i j}
$$

Now setting $i=j=1$ gives the first Cauchy-Riemann equation,

$$
\begin{gathered}
2 \frac{\partial X_{1}}{\partial x_{1}}=\frac{\partial X_{1}}{\partial x_{1}}+\frac{\partial X_{2}}{\partial x_{2}} \\
\frac{\partial X_{1}}{\partial x_{1}}-\frac{\partial X_{2}}{\partial x_{2}}=0
\end{gathered}
$$

And on the other hand, setting $i=1, j=2$ gives the second Cauchy-Riemann equation,

$$
\frac{\partial X_{1}}{\partial x_{2}}+\frac{\partial X_{2}}{\partial x_{1}}=0
$$

Thus, if a function $X$ is differentiable, its derivative is continuous and it satisfies the Cauchy-Riemann equations, it is complex analytic on the part of the plane that satisfies the Cauchy-Riemann equations. With any complex analytic function the real and imaginary parts are harmonic conjugate.[59] Specifically, since the components 
of a conformal vector field $X_{1}+\mathrm{i} X_{2}$ are complex analytic and satisfy $\nabla X_{1} \cdot \nabla X_{2}=0$, $\left\|\nabla X_{1}\right\|^{2}=\left\|\nabla X_{2}\right\|^{2}$, and Laplace's equation $\nabla^{2} X_{1}=\nabla^{2} X_{2}=0$, the components of the vector field $\left(X_{1}, X_{2}\right)$ are perpendicular, but furthermore, $\nabla X_{2}$ is $90^{\circ}$ anticlockwise from $\nabla X_{1}$ and equal in magnitude. (Figure 9.3)

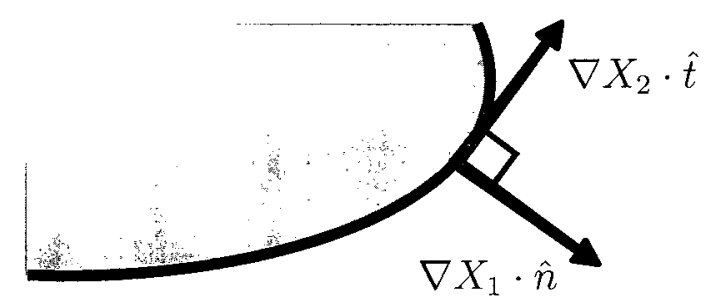

Figure 9.3: Derivatives of the components of a conformal vector field at the boundary

Recall the conductivity equation $\nabla \cdot \sigma \nabla \phi=0$ and that a Dirichlet-to-Neumann map $\Lambda_{\sigma}$ takes a potential $\phi$ restricted to the boundary of the domain $\partial \Omega$ and maps it to a current density $J=\sigma \partial \phi / \partial \hat{n}$ where $\hat{n}$ is the outward unit normal on the boundary. In particular, the Dirichlet-to-Neumann map $\Lambda_{1}$ is a solution for Laplace's equation with conductivity $\sigma=1$. In EIT, this map $\Lambda_{1}:\left.\phi\right|_{\partial \Omega} \mapsto J$ is solved using an Finite Element Method (FEM) over any arbitrarily shaped domain. This same map can be used to solve Laplace's equation such that $\Lambda_{1}:\left.\phi\right|_{\partial \Omega} \mapsto X$, where the operator $\Lambda_{1}$ is no longer acting as a transconductance operator but instead as a voltage-to-movement operator.

The Dirichlet-to-Neumann operator $\Lambda_{1}$ is used to convert Dirichlet data on the boundary $\left.X_{1}\right|_{a \Omega}$ to Neumann data normal to the boundary $\left(\nabla X_{1} \cdot \hat{n}\right)$ which is the 
same as the tangential Neumann boundary data for $X_{2}\left(\nabla X_{2} \cdot \hat{t}\right)$.

$$
\begin{aligned}
\left.\Lambda_{1} \phi\right|_{\partial \Omega} & =\nabla \phi \cdot \hat{n}=\frac{J_{n}}{\sigma} \\
\left.\Lambda_{1} X_{1}\right|_{\partial \Omega} & =\nabla X_{1} \cdot \hat{n}=\nabla X_{2} \cdot \hat{t}
\end{aligned}
$$

where $\hat{t}$ is the tangent vector and $\hat{n}$ is the normal vector on the boundary $\partial \Omega$.

Knowing the tangential derivative of $X_{2}$ at the boundary is essentially the same as knowing the Dirichlet data for $X_{2}$ since it can be integrated along the boundary.

$$
\nabla X_{2} \cdot \hat{t}=\frac{\partial X_{2}}{\partial s}
$$

where $s$ is the anti-clockwise arc length along the boundary $\partial \Omega$.

Combining (9.5) (9.6) and integrating along $d s$ gives

$$
X_{2}=\int_{0}^{s} \Lambda_{1} X_{1} d s+\text { const }
$$

such that $X_{1}$ and $X_{2}$, within the domain $\Omega$, are determined by values on the boundary $\partial \Omega$, and the boundary values of a conformal $X$ are exactly those with

$$
X_{2}-\int_{0}^{s} \Lambda_{1} X_{1} d s=\text { const }
$$

or

$$
\frac{\partial}{\partial s} X_{2}-\Lambda_{1} X_{1}=0
$$


and in matrix form

$$
\left[\begin{array}{ll}
-\Lambda_{1} & L_{1}
\end{array}\right]\left[\begin{array}{l}
X_{1} \\
X_{2}
\end{array}\right]=0
$$

where $L_{1}$ is the difference operator along the boundary $\frac{\partial}{\partial s}$.

Thus, $X_{1}$ can be specified arbitrarily on the boundary, and its tangential derivative $d X_{2} / d s$ is determined. $X_{2}$ is the solution of a Neumann problem for Laplace's equation and is determined up to a constant. Constants added to $X_{1}$ and $X_{2}$ correspond to a translation, which would not be expected in EIT data.

This means that, in the discrete setting of the FEM, specifying a distortion on the boundary nodes will give a unique conformal map. In the FEM setting, the infinitesimal vector field $X$ becomes a vector that translates each node of the finite element mesh. Simply applying the vector field to the mesh will result in a globally conformal transformation, but locally about the nodes, the angles between edges joining elements will have changed. The vector field $X$ is continuous, and therefore, an accurate application of the field will "bend" the shape of the mesh elements to maintain the local angles about each node.

\subsection{Summary and Discussion}

This work describes the mathematical constraints under which conformal motions occur in isotropic EIT images. The results show that for any boundary deformation a corresponding conformal map can be found, and hence, any boundary motion can be decomposed into its conformal and non-conformal components.

This information would be useful when evaluating the performance of algorithms 
that correct for boundary movement when that change is known because these algorithms can not detect the conformal component of the boundary movement and therefore, should not be penalized for their lack of performance on the conformal component of boundary movements.

This approach could be applied, rather than the one presented in Chapter 6, to take the true deformation of the phantom's boundary from the photographs and decompose it into its conformal and non-conformal components. The work here suggests that the conformal component should not match the reconstructed electrode movement while the non-conformal component should match closely up to a limit where the FEM mesh structure and boundary deformation near the translated electrodes interferes with the free movement of the electrodes. This requires an implementation of the conformal/non-conformal decomposition, which has not been achieved at this time.

Figure 9.2 used the forward solver implemented in the Electrical Impedance and Diffuse Optics Reconstruction Software (EIDORS) package, version 3.3. The shape deformations were implemented as separate code. 


\section{Chapter 10}

\section{Applied Electrode Models}

Electrical Impedance Tomography (EIT) applies current and measures the resulting voltage on the surface of a target. In biomedical applications, this current is applied, and voltage is measured through electrodes attached to the surface. Electrode models represent these connections in the reconstruction of the conductivity image, but changes in the contact impedance or boundary relative to the electrode area can introduce artifacts in the reconstructed image. The effects of boundary deformation and contact impedance variation are investigated here.

Mathematical proofs of solution existence and uniqueness for EIT initially employed a Continuum Model for the electrodes, which implied complete knowledge of all boundary data. [30, 31] Effectively, it was an infinite set of infinitesimally small electrodes without gaps between them. The proof was later extended to allow for regular gaps in the boundary data (Gap Model), but experimental data did not match forward simulations using this model. The Gap Model was insufficient because simplistically, electrodes are conductive, and thus, the boundary surface attached to 
a single electrode might have a uniform voltage thus leading to the Shunt Electrode Model (SEMI). The mathematical model was further refined as the Complete Electrode Model (CEM) which allows an additional complex impedance for each electrode and models the metal electrode, conductive gel and chemical interaction at the skin-electrode interface.[32, 33] (Figure 10.1)

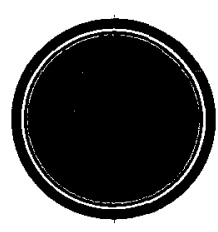

CM

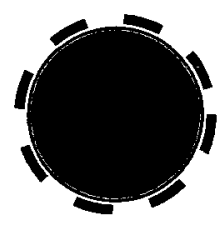

GM

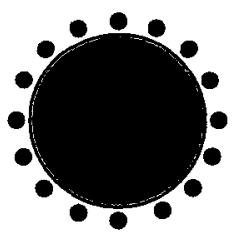

SEM

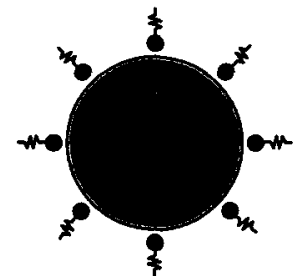

CEM

Figure 10.1: Electrode models used in mathematical proofs: CM - Continuous Model, GM - Gap Model, SEM - Shunt Electrode Model, CEM - Complete Electrode Model

As described in Chapter 3, the Finite Element Method (FEM) is used in the numerical solution of EIT images. The simplest possible electrode model to implement in the FEM is the Point Electrode Model (PEM) which applies current and measures voltage at single nodes on the boundary. The PEM does not consider the geometry or contact impedance of an electrode. An alternative electrode model mirrors the mathematical SEM in forcing all nodes associated with an electrode to the same voltage. The SEM is appropriate when contact impedances are so small that the matrices become ill-conditioned (contact impedance is in the denominator), and thus, forcing nodes to the same voltage is a reasonable means of making the problem more tractable.[3] Finally, to reconstruct accurate images from real in vivo data, an accurate electrode model is required, and thus, the CEM is generally preferred.[32] (Figure 10.2, 10.3) In solving the inverse problem for contact impedance, previous reconstructions of the CEM contact impedance under homogeneous conductivity 
conditions have been successful. $[60,61,62]$

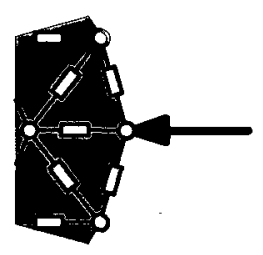

PEM

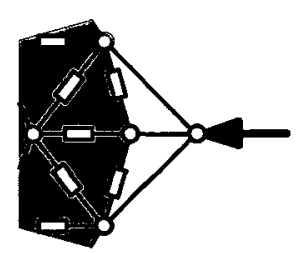

SEM

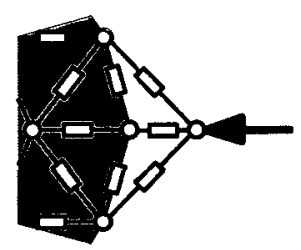

CEM

Figure 10.2: Electrode models implemented in a Finite Element Model, viewed as a resistor network: PEM - Point Electrode Model, SEM - Shunt Electrode Model, CEM - Complete Electrode Model

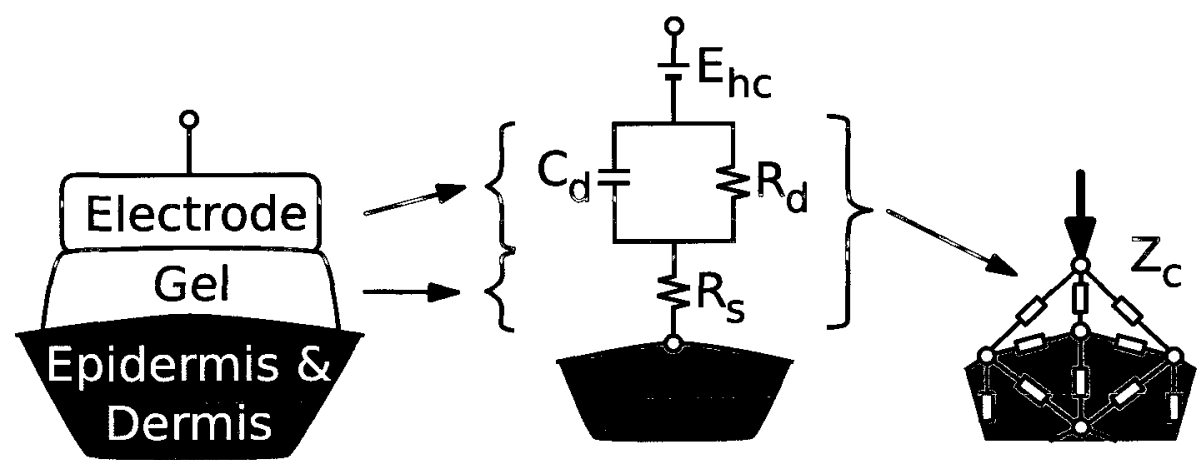

Figure 10.3: Generalized electrode model and its relationship to the Complete Electrode Model, adapted from [14,63], $E_{h c}$ electrode half-cell potential, $R_{d} C_{d}$ electrode impedance, $R_{s}$ gel related impedance, $R_{s}+\left(R_{d} \|-\mathrm{i} / \omega C_{d}\right)=Z_{c}$ contact impedance.

\subsection{Contact Impedance and Electrode Area}

Electrode contact impedance is commonly defined in units of impedance and area $\left(\Omega \cdot \mathrm{m}\right.$ in $2 \mathrm{D}, \Omega \cdot \mathrm{m}^{2}$ in $\left.3 \mathrm{D}\right)$. In EIT, conformal movements of the boundary, such as dilation, occur with no change in the reconstructed image when using a PEM [8].

For a CEM spanning multiple edges on the FEM mesh boundary, there must be a distribution of the contact impedance amongst those edges. One method is to use 
a linear expansion function that assigns the contact impedance based on the length between nodes in two dimensions or area of a boundary element in three dimensions as in [64].

If contact impedance is specified in $\Omega \cdot \mathrm{m}^{2}$ or $\Omega \cdot \mathrm{m}$, the location of the FEM nodes for the electrode must accurately reflect the total area of the electrode to achieve the correct overall electrode impedance. This is not trivial because the FEM mesh's node locations do not necessarily represent the physical boundary; conformal deformations can be applied to the FEM mesh without affecting the voltage at the nodes of the FEM.

An in vivo deformation could be decomposed into independent conformal and non-conformal components, but the electrodes themselves are generally fabric or plastic backed and thus, can not stretch as the deformation occurs. Therefore, it is likely that, if a conformal deformation such as a dilation occurs, the area of the electrodes will not change, and the result will be artifacts in the image due to the change in the boundary definition. One would expect that an error in electrode area is the inverse of an error in specifying the contact impedance because the overall electrode impedance is a function of contact impedance over area. Figure 10.4 shows this to be incorrect; increasing electrode area has the same effect as increasing contact impedance.

Simulations were performed on a two-dimensional circular tank with homogeneous conductivity (33439 elements, 16 CEM $0.2 \mathrm{~m}$ electrodes, background conductivity of $1 \mathrm{~S} / \mathrm{m}$ ) and circular and rectangular targets (conductivity $2 \mathrm{~S} / \mathrm{m}$ ). The measurements were reconstructed using a course mesh (7207 elements, 16 CEM 0.2m electrodes, Tikhonov single step inverse solver $\mu=1 \mathrm{e}-5$, Tikhonov image prior). Im- 


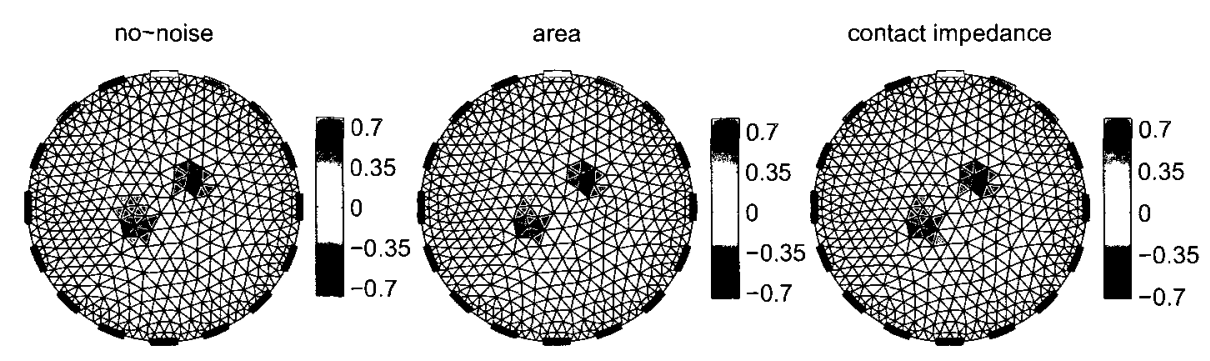

Figure 10.4: Electrode area and contact impedance artifacts are quite similar: (left) reconstruction, no noise; (middle) reconstruction, electrode areas reduced to $1 / 4$, $\mathrm{AAM}_{n}=5.5$; (right) reconstruction, contact impedances reduced to $1 / 16, \mathrm{AAM}_{n}=$ 7.04

age artifacts were quantified using the Artifact Amplitude Measure (AAM), the sum of the squared normal error of an image's conductivity elements $\mathbf{c}_{1}$, where error is relative to a no-noise conductivity reconstruction $\mathbf{c}_{0}$.

$$
\mathrm{AAM}_{n}=\sum\left[\frac{\mathbf{c}_{1}-\mathbf{c}_{0}}{\mathbf{c}_{0}}\right]^{2}
$$

The behaviour of the PEM and CEM were explored under two types of conformal deformation: a 10\% dilation, and a more complex deformation defined in Figure 10.5. The PEM was not affected by conformal changes, whether the electrode model fixed the area or it changed to match the boundary. For the CEM, changes that were symmetric, e.g. dilation, did not result in significant artifacts if the electrode deformed with the boundary change. When the area of the CEM was fixed and a dilation occurred, "ringing" artifacts were observed. (Figure 10.4, middle image) When the CEM was deformed in a complex conformal manner, deformation of the reconstructed image was observed as a form of artifact.(Table 10.1) 


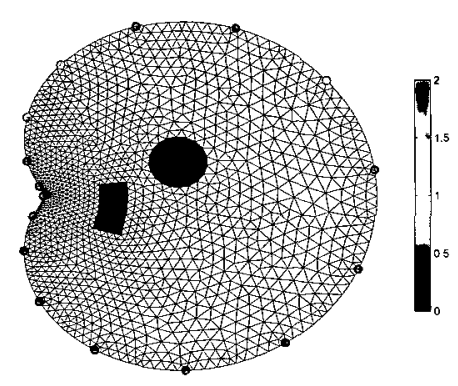

Figure 10.5: Complex conformal deformation used in electrode model deformation simulations: $z=x+\mathrm{i} y ; z \rightarrow z+0.4 z^{2}$

Table 10.1: Electrode Model Behaviour under Deformation

\begin{tabular}{ccccc}
\hline & \multicolumn{2}{c}{ Deformation } & & \\
\cline { 2 - 3 } Model & Domain & Electrode & $\mathrm{AAM}_{n}$ & Comment \\
\hline PEM & dilation & matching & 0 & \\
& complex & & 0.0807 & \\
& dilation & fixed & 0 & \\
CEM & dilation & matching & 0.0010 & \\
& complex & & 2.013 & artifacts (deformed) \\
& dilation & fixed & 5.5 & artifacts (ringing) \\
\hline
\end{tabular}

\subsection{Contact Impedance Variation}

Depending on current injection patterns, there can be a significant requirement for correct specification of electrode impedance when an absolute image reconstruction is to be attempted. [65] For difference imaging, two "frames" of data are subtracted to form an image which removes the need to resolve the component of both contact and internal conductivity common to the two "frames". It is commonly assumed in the reconstruction process that the contact impedance remains constant, and thus, all measurement changes are due to internal conductivity changes. As electrodes age, their contact impedance changes, but changes that are not correlated will be 
minimized by difference imaging with frames taken at short intervals. (Typical EIT systems, such as the Goe-MF II EIT system, acquire at 13 frames-per-second.) It was found that changes that were correlated did not affect the reconstruction with PEMs; while for CEMs, ringing was observed when impedances decreased which was analogous to the fixed area electrodes under a dilation deformation. Impedance increases had a minimal effect. Independent of the electrode model, a large change in contact impedance for a single electrode appeared in the reconstructed image as an artifact near the electrode. The effects were less clear where contact impedance changed in a manner that was uncorrelated amongst the electrodes. For the purposes of this work, this will be termed "electrode noise."

Simulations of the effect of uncorrelated electrode contact impedance changes, as might be found with in vivo electrodes, were performed. For comparison, a reconstruction with no electrode noise or measurement noise and a similar reconstruction with only measurement noise (50dB SNR) were simulated. (Figure 10.6)
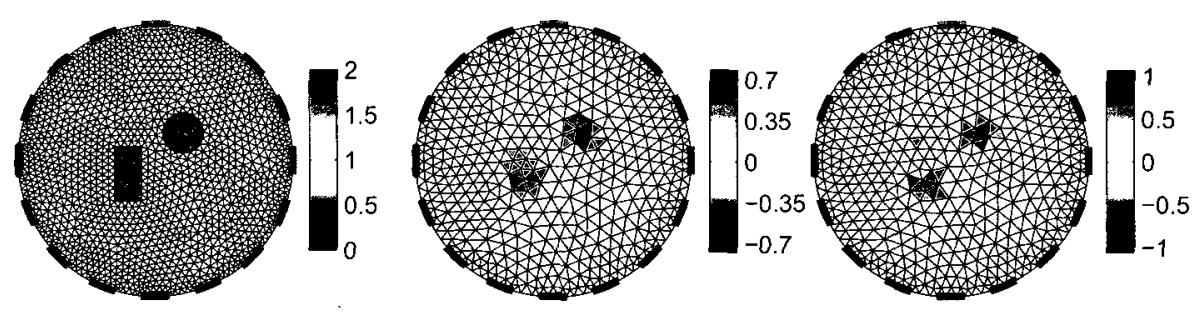

Figure 10.6: Difference image of two targets illustrating the effect of measurement noise on reconstruction quality: (left) forward model; (middle) reconstruction, no noise, $\mathrm{AAM}_{n}=0$; (right) reconstruction, $50 \mathrm{~dB} \mathrm{SNR}, \mathrm{AAM}_{n}=7.0$

These initial reconstructions were compared to reconstructions with only electrode noise at various levels (Figure 10.7). Electrode contact impedance $z_{c}[\Omega \cdot \mathrm{m}]$ 
(i.e. electrode noise) was assigned using an exponential Gaussian distribution

$$
z_{c}=10^{\mathcal{N}}\left(\mu, \sigma^{2}\right)
$$

where $\mathcal{N}$ is a Gaussian distribution with a given mean $\mu$ and variance $\sigma^{2}$.

With minimal electrode noise $\mu=0$ and $\sigma^{2}=0.25(\simeq 1 \Omega \cdot \mathrm{m})$, no noticeable reconstruction artifacts were observed, similar to the no noise reconstruction of Figure 10.6. With an increase in electrode contact impedance variability, $\mu=-0.75$ and $\sigma^{2}=1.5$, image artifacts resembling those seen in the presence of measurement noise (50dB SNR) were observed. Electrode impedances varying such that $\mu=-1.5$ and $\sigma^{2}=3$ resulted in significant artifacts throughout the image.
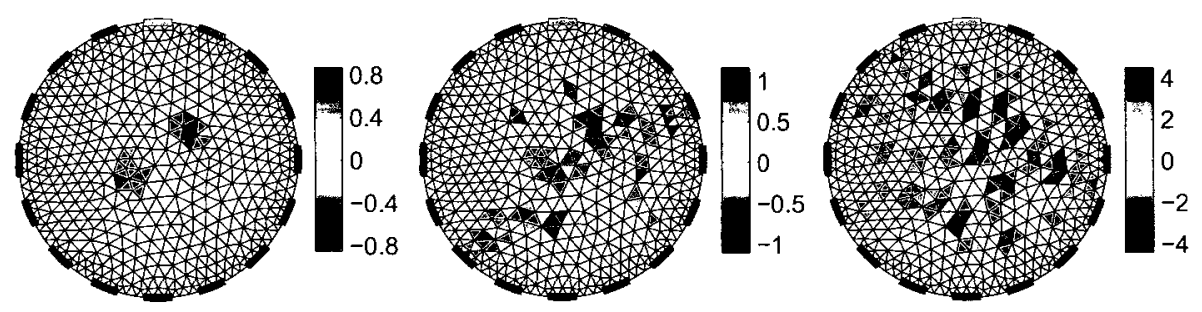

Figure 10.7: Difference image of two targets illustrating the effect of increasing electrode noise on reconstruction quality; (left) $\mu=0, \sigma^{2}=0.25, \mathrm{AAM}_{n}=5.9$; (middle) $\mu=-0.75, \sigma^{2}=1.5, \mathrm{AAM}_{n}=24.5 ;$ (right) $\mu=-1.5, \sigma^{2}=3, \mathrm{AAM}_{n}=$ 74.1

It would be reasonable to expect that, as electrode contact impedance variability increases, the magnitude and quantity of image artifacts increases whether the electrode's impedance has increased or decreased. To explore this supposition, the effect of differing electrode noise distributions on mean artifact amplitude was simulated in Figure 10.8. At each step, a specific mean and variance $\left(-3 \leq \mu \leq 3\right.$ and $\left.\sigma^{2}=|\mu|\right)$ were used to draw 100 electrode contact impedance configurations. These configura- 
tions were simulated and the mean and variance of the AAM in the reconstructions was plotted (top). The contact impedance distribution was also plotted (bottom). Somewhat unexpectedly, increases in contact impedances resulted in no observable artifacts in the reconstructed image. This was confirmed by holding the variance constant and varying the mean of the contact impedance distribution $(-3 \leq \mu \leq 3$ and $\left.\sigma^{2}=1\right)$. (10.9)
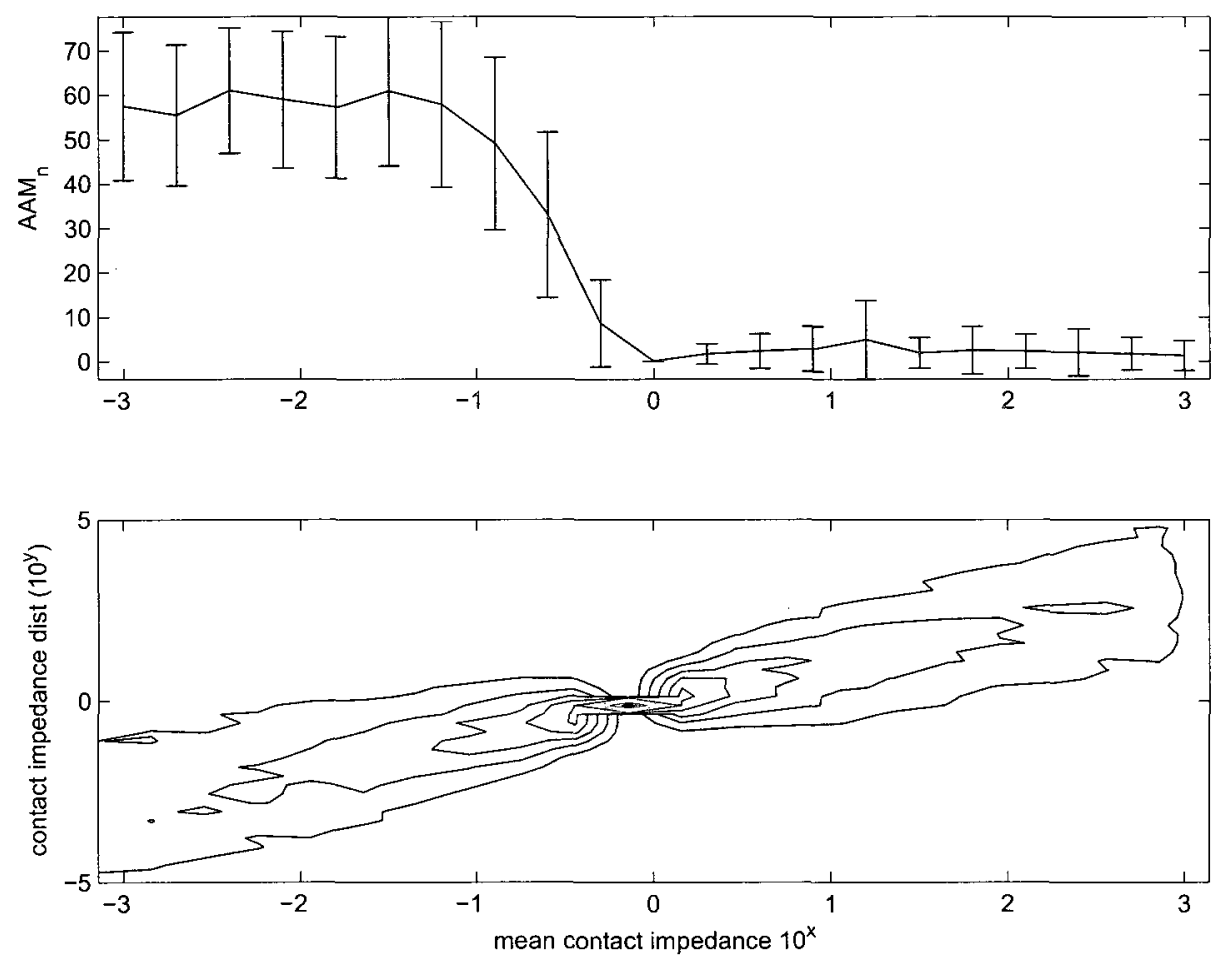

Figure 10.8: Electrode noise distributions plotted versus artifact amplitude, illustrating that increasing contact impedance does not introduce artifacts.

\subsection{Summary and Discussion}

This work develops a method for understanding and quantifying the effect of errors in electrode area and contact impedance that occur in two-dimensional reconstructions 

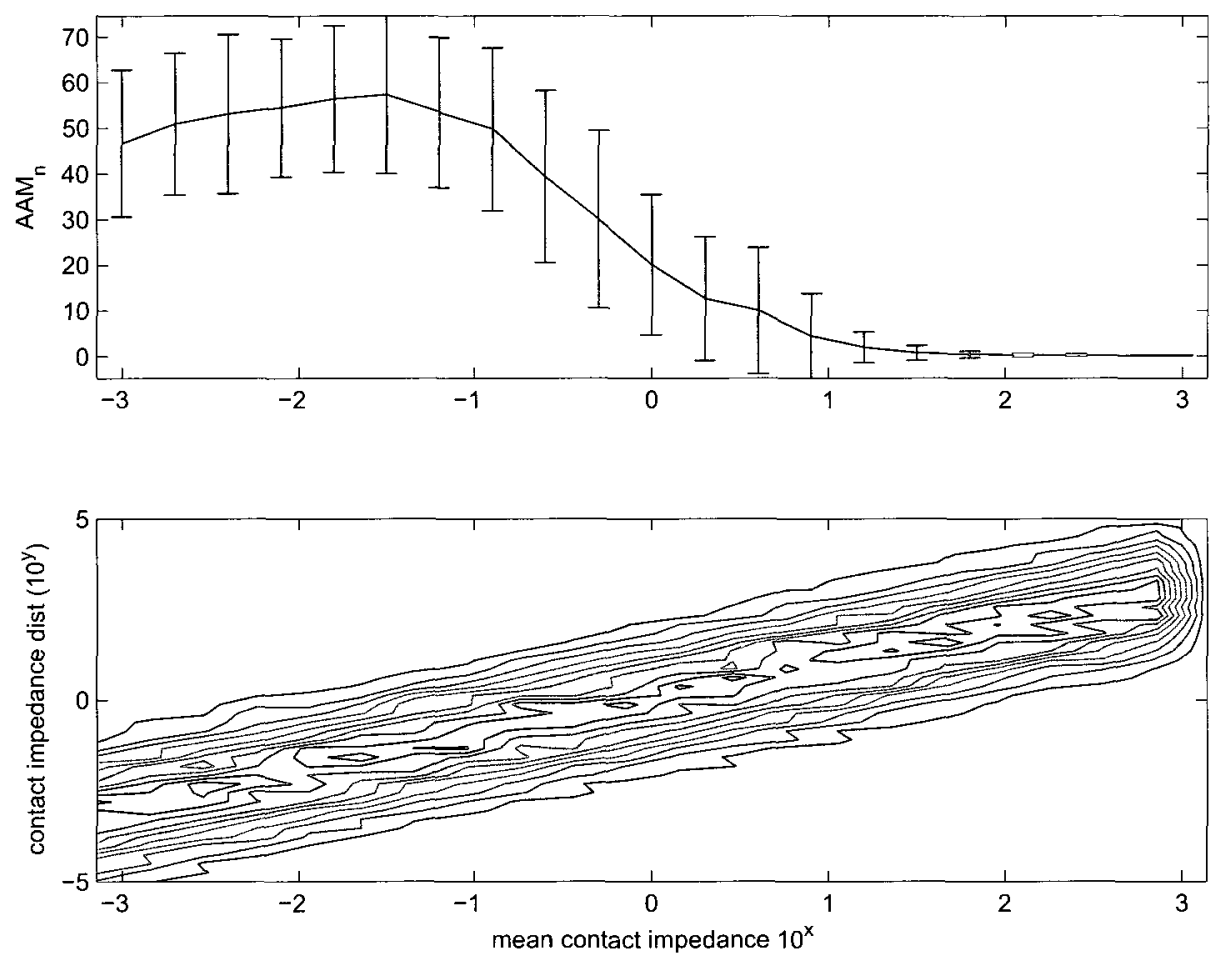

Figure 10.9: Electrode noise distributions plotted versus artifact amplitude, illustrating that increasing contact impedance does not introduce artifacts. 
when the boundary is deformed. Results show that the CEM produces artifacts when conformal deformations are applied. The results obtained for contact impedance variation simulations generally agree with previously published results [66] which indicated that variation of as little as $20 \%$ can result in an image that has artifacts significant enough to render the image "almost meaningless."

In the context of electrode displacement correcting algorithms using PEM or CEM electrodes, as in [51], where electrodes are translated without rotation, scaling or other deformation, the elements of the FEM associated with the electrode maintain their relative geometry, and no modification of the contact impedance distribution is required to maintain the original current density across the electrode. Restricting the movement of the electrode to translation simplifies the electrode movement algorithm but introduces unwanted boundary distortions on the elements adjoining the electrode that restricts the range of detectable deformations as the size of the mesh elements on the boundary are reduced. This represents a significant drawback to this technique if finer FEM meshes along the boundary are to be implemented in the future.

Typically, these electrode related image-reconstruction issues do not arise in simulation because a common electrode model is used in the forward problem and its inverse solution. The electrode contact impedance is assumed constant throughout and therefore, cancels in difference imaging. In vivo changes in contact impedance due to electrode movement do occur to some degree, and simulations that do not apply appropriate variation to the contact impedances are likely to get optimistic results. The results show that, in most cases, applying some level of measurement noise may have the same effect as contact impedance variation and should be an 
appropriate approximation.

The unexpectedly artifact free reconstructions when contact impedance increases might be explained by considering the electrode model as a resistor network attached to the FEM, itself a low impedance resistor network. Large contact impedances mean that the voltage measured at the electrode is approximately the average of the boundary voltage connected to the electrode; however, a small contact impedance will result in an electrode voltage that is highly dependent on the surrounding conductivity. In this environment, electrode voltages measured across small contact impedances will be heavily affected by reconstructed conductivity artifacts near the boundary and are therefore more likely to introduce these artifacts in the inverse problem.

In general biomedical and industrial applications, achieving a minimal contact impedance is desirable to maximize measurement sensitivity. The EIT simulations described in Section 10.2 show that, with contact impedance variability, reconstruction artifacts can be a significant factor in image quality as contact impedance is reduced. Increasing the electrode contact impedance or simultaneously reconstructing the contact impedance and interior conductivity through some form of regularization may mitigate these effects. Further in vivo studies of electrode contact impedance under boundary movement are required to determine if electrode impedances do in fact vary by a sufficient amount to warrant further attempts at mitigating the effect of impedance variability.

The electrode models, forward models and reconstruction algorithms were those implemented in the Electrical Impedance and Diffuse Optics Reconstruction Software (EIDORS) package, version 3.3. The shape deformations and analysis were implemented as separate code. 


\section{Chapter 11}

\section{Conclusion and Future Work}

This thesis explored the effect of specific shape deformations on two-dimensional Electrical Impedance Tomography (EIT) through theoretical explorations and the use of simulation and phantom studies. It describes conformal and non-conformal vector fields and develops their application to electrode movement and boundary distortion in EIT.

An EIT reconstruction technique that adapts to boundary deformation was evaluated in vitro through the construction of a two-dimensional, deformable phantom. The algorithm was shown theoretically to be incapable of discovering a specific class of deformations, the conformal motions. These conformal deformations were explored mathematically in an EIT context, and a differential interpretation of these results was provided in a form more recognizable to the engineering community. The effect of electrode models under these deformations was explored, and it was found that the Complete Electrode Model (CEM) can cause significant artifacts when the electrode does not deform in the same manner as the boundary as is likely to occur 
in vivo. It was also shown that contact impedance reductions resulted in significant artifacts while contact impedance increases resulted in artifact free images.

Results, both in simulation and with experimental data, suggest that, with nonconformal mappings, electrode movement and boundary distortions can be reconstructed based on conductivity changes alone which reduces image artifacts in the process.

These results have been published in the proceedings of the $g^{\text {th }}$ International Conference on Biomedical Applications of Electrical Impedance Tomography, Dartmouth, USA (EIT 2008) and the $10^{\text {th }}$ International Conference on Biomedical Applications of Electrical Impedance Tomography, Manchester, UK (EIT 2009).[8][9] Software modifications and updates supporting this work have been contributed to the Electrical Impedance and Diffuse Optics Reconstruction Software (EIDORS) package [10] under the GNU General Public License.

\subsection{Recommendations}

Some recommendations coming from this body of work are as follows:

- Be aware that conformal deformations are possible in two-dimensional EIT and that, therefore, distortions of the reconstructed conductivities may not match expected shapes while at the same time not introducing obvious artifacts into the image by which the situation might be recognized. These distortions will be due to the conformal element of a deformation. An exception to this occurs with images reconstructed using a CEM where images containing

- ringing are indicative of symmetric errors in contact impedance or area 
which in turn may indicate dilation if the electrode area is fixed, or

- distortions are indicative of conformal inversions which may not be intuitive.

- Measurement noise is an appropriate method of approximating general difference errors between EIT frames for electrode area and contact impedance changes in simulation studies.

- The two-dimensional reconstructions presented here are an approximation of a three-dimensional problem. The fact that this work has focused solely on two-dimensional reconstructions is not meant to imply that this is the best or most appropriate technique. Three-dimensional reconstructions should be the ultimate goal.

\subsection{Future Work}

There are a number of directions in which to further explore the themes of this work:

- Develop code to separate a known shape deformation into conformal and nonconformal components. Using this technique, it would be possible to validate electrode movement algorithms against a theoretical limit. The mathematical background provided in Chapters 8 and 9 should be sufficient, but an implementation has not successfully been completed at this time. The phantom from Chapter 6 could be used to find how closely the non-conformal component of movement is reconstructed. 
- Use the phantom developed in Chapter 6 to investigate deformations that are explicitly conformal. Determine if the expected change in isotropic conductivity occurs in vitro as indicated in Chapter 8.

- Improve the Fourier series boundary fitting algorithm to allow for angular electrode movement.

- Extend this work into three dimensions. Conformal motions are significantly limited in their degrees of freedom in three dimensions when compared to two dimensions.

- Explore whether it is possible to detect conformal boundary changes

- given fixed size electrodes (Chapter 10), or

- by the change in conductivity related to the conformal motion (Chapter 8$)$.

- Create some form of regularized combination of contact impedance, conductivity, and electrode movement reconstruction. It may be possible to obtain contact impedances without requiring a homogeneous medium. This would be another key piece in reducing the artifacts in in vivo conductivity reconstruction that may eventually allow in vivo three-dimensional nonlinear and absolute reconstructions.

- Explore whether high impedance electrodes are a practical means to allow reconstructions exhibiting fewer artifacts.

These studies were based on a linear approximation of the dependence of the transfer impedance data on both the conductivity and shape. Most in vivo EIT 
studies assume a linear approximation and typically reconstruct time or frequency difference images even though the forward problem is non-linear. One reason that is given for why a non-linear forward solution gives improved images on in vitro tank data but fails to deliver an improvement on in vivo reconstruction, is that the errors caused by an inaccurate knowledge of boundary shape are greater than the error in using a linear approximation. This work holds out the hope that, with the correction of the boundary shape and electrode positions, using the EIT data will be sufficient for non-linear and accurate absolute EIT reconstruction of clinical data at sometime in the future.

A limitation of the method of detecting boundary movement used in this work is that the subject is assumed to be isotropic. While this is a reasonable approximation for the lung, it is not true of muscle tissue or flowing blood. The effect of parts of the domain being anisotropic, possibly in a predictable orientation, is an interesting topic for further investigation. 
Appendices 


\section{Appendix A}

\section{Vector Calculus}

A brief review of some relevant vector calculus is contained in this appendix, summarized from [67]. In this section, $\boldsymbol{F}$ refers to a vector field $\left(F_{x} \hat{i}+F_{y} \hat{j}+F_{z} \hat{k}\right)$, and $f$ refers to a scalar field $f(x, y, z) . \hat{i} \hat{j} \hat{k}$ are the standard Cartesian unit vectors along the $\mathrm{x}-, \mathrm{y}-$, and $\mathrm{z}$-axes respectively. The vector fields themselves can be written as matrices.

$$
\mathbf{F}=\left[\begin{array}{c}
F_{x} \\
F_{y} \\
F_{z}
\end{array}\right]
$$

\section{A.1 Determinant}

The determinant can be found for any square matrix. If the matrix is taken as a linear transformation matrix, the determinant is the scaling factor of the transformation. A matrix is invertible if and only if the determinant of the matrix is non-zero. 
The determinant of a two-by-two matrix is

$$
\operatorname{det}(\mathbf{A})=\left|\begin{array}{ll}
a & b \\
c & d
\end{array}\right|=a d-b c
$$

In two dimensions, the determinant is the area of a parallelogram where a corner is anchored at the origin and the sides are defined by the corners $(a, b)$ and $(c, d)$. (Figure A.1) This geometric meaning can be extended to three dimensions (resulting in three-by-three matrix determinants) and beyond.

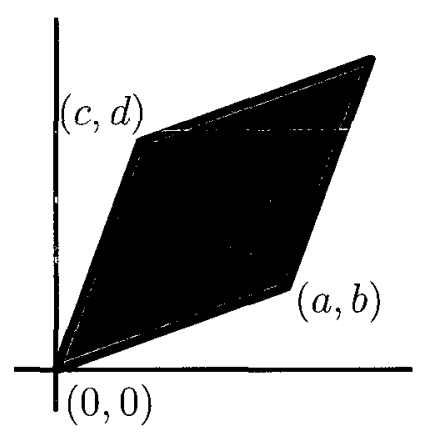

Figure A.1: Parallelogram; corners at $(a, b)$ and $(c, d)$, anchored at $(0,0)$

\section{A.2 Vector Products}

The dot-product $(\mathbf{A} \cdot \mathbf{B})$ and cross-product $(\mathbf{A} \times \mathbf{B})$ are two common operators used in vector calculus.

In three dimensions,

$$
\mathbf{F} \cdot \mathbf{G}=\mathbf{F}^{\top} \mathbf{G}=\left[\begin{array}{lll}
F_{x} & F_{y} & F_{z}
\end{array}\right]\left[\begin{array}{c}
G_{x} \\
G_{y} \\
G_{z}
\end{array}\right]=F_{x} G_{x}+F_{y} G_{y}+F_{x} G_{y}
$$




$$
\begin{gathered}
F \times \mathbf{G}=\left|\begin{array}{ccc}
\hat{i} & \hat{j} & \hat{k} \\
F_{x} & F_{y} & F_{z} \\
G_{x} & G_{y} & G_{z}
\end{array}\right| \\
\mathbf{F} \times \mathbf{G}=\left(F_{x} G_{z}-F_{z} G_{y}\right) \hat{i}-\left(F_{x} G_{z}-F_{z} G_{x}\right) \hat{j}+\left(F_{x} G_{y}-F_{y} G_{x}\right) \hat{k}
\end{gathered}
$$

The Del operator $\nabla$ is a vector of the partial derivatives of some unspecified vector field

$$
\nabla=\left[\begin{array}{c}
\frac{\partial}{\partial x} \\
\frac{\partial}{\partial y} \\
\frac{\partial}{\partial z}
\end{array}\right]
$$

and is used in the vector calculus notation for gradient, divergence and curl.

\section{A.3 Gradient}

Gradient is a vector field representing the slope of a scalar function.

In three dimensions, gradient of a scalar field $f$ is

$$
\nabla f=\left[\begin{array}{c}
\frac{\partial}{\partial x} \\
\frac{\partial}{\partial y} \\
\frac{\partial}{\partial z}
\end{array}\right] f=\frac{\partial f}{\partial x} \hat{i}+\frac{\partial f}{\partial y} \hat{j}+\frac{\partial f}{\partial z} \hat{k}
$$

\section{A.4 Divergence}

Divergence is a scalar field representing how much a vector field within a region is behaving as a source or sink. Divergence has units of $[\cdot / \mathrm{m}]$. 
In three dimensions, divergence of a vector field $\mathbf{F}$ is

$$
\nabla \cdot \mathbf{F}=\left[\begin{array}{ccc}
\frac{\partial}{\partial x} & \frac{\partial}{\partial y} & \frac{\partial}{\partial z}
\end{array}\right]\left[\begin{array}{c}
F_{x} \\
F_{y} \\
F_{z}
\end{array}\right]=\frac{\partial F_{x}}{\partial x}+\frac{\partial F_{y}}{\partial y}+\frac{\partial F_{z}}{\partial z}
$$

A few identities with respect to divergence are as follows:

$$
\begin{gathered}
\nabla \cdot(\nabla \times \mathbf{F})=0 \\
\nabla \cdot(\mathbf{F} \times \mathbf{G})=(\nabla \times \mathbf{F}) \cdot \mathbf{G}-\mathbf{F} \cdot(\nabla \times \mathbf{G}) \\
\nabla \cdot(g \mathbf{G})=(\nabla g) \cdot \mathbf{F}+g(\nabla \cdot \mathbf{F})
\end{gathered}
$$

\section{A.5 Curl}

Curl expresses the right-hand rule, finding the rotation of a vector field where the axis of rotation is the direction of the curl vector and magnitude of rotation is the magnitude of the curl vector. Curl has units of $[\cdot / \mathrm{m}]$.

In three dimensions, curl of a vector field $\mathbf{F}$ is

$$
\begin{gathered}
\nabla \times \mathbf{F}=\left|\begin{array}{ccc}
\hat{i} & \hat{j} & \hat{k} \\
\frac{\partial}{\partial x} & \frac{\partial}{\partial y} & \frac{\partial}{\partial z} \\
F_{x} & F_{y} & F_{z}
\end{array}\right| \\
\nabla \times \mathbf{F}=\left(\frac{\partial F_{z}}{\partial y}-\frac{\partial F_{y}}{\partial x}\right) \hat{i}+\left(\frac{\partial F_{x}}{\partial z}-\frac{\partial F_{z}}{\partial x}\right) \hat{j}+\left(\frac{\partial F_{y}}{\partial x}-\frac{\partial F_{x}}{\partial y}\right) \hat{k}
\end{gathered}
$$


A pair of identities with respect to curl are as follows:

$$
\begin{gathered}
\nabla \times(\nabla f)=0 \\
\nabla \times(\nabla \times \mathbf{F})=\nabla(\nabla \cdot \mathbf{F})-\nabla^{2} \mathbf{F}
\end{gathered}
$$

In general, Stokes' Theorem (A.15) relates a surface integral of the curl of $\mathbf{F}$ to a line integral of $\mathbf{F}$ on the boundary. In two dimensions, this is Green's Theorem.

\section{A.6 Laplacian}

The Laplacian is a second-order differential operator. It shows up as the diffusion of a scalar field towards an equilibrium.

$$
\nabla^{2} f=\nabla \cdot \nabla f
$$

\section{A.7 $2 \times 2$ Matrix Inverse}

For a two-by-two matrix,

$$
\mathbf{A}=\left[\begin{array}{ll}
a & b \\
c & d
\end{array}\right]
$$

where the matrix $\mathbf{A}$ is non-singular such that its determinant is non-zero $(|\mathbf{A}| \neq 0)$, the inverse of the matrix is

$$
\mathbf{A}^{-1}=\frac{1}{a d-b c}\left[\begin{array}{cc}
d & -b \\
-c & a
\end{array}\right]
$$

where the denominator $a d-b c$ is the determinant of the matrix $\mathbf{A}$. 


\section{Appendix B}

\section{Generalized Tikhonov Derivation}

This section presents the derivation of a solution for the single step Tikhonov regularization scheme. The derivation is not new but is presented here as an application of the level of matrix algebra required to understand the technical details of Electrical Impedance Tomography (EIT) implementations.

A few basic matrix algebra identities are required in these solutions:

$$
(\mathbf{J} \mathbf{c})^{\top}=\mathbf{c}^{\top} \mathbf{J}^{\top}, \quad \mathbf{c}^{\top} \mathbf{J}^{\top} \mathbf{z}=\mathbf{z}^{\top} \mathbf{J} \mathbf{c}, \quad(\mathbf{J} \mathbf{c})^{\top} \mathbf{z}=\mathbf{z}^{\top} \mathbf{J} \mathbf{c}, \quad \mathbf{y}^{\top} \mathbf{z}=\mathbf{z}^{\top} \mathbf{y}
$$

An excellent general resource for working with matrix algebra is [68]. 


\section{B.1 Least Squares Solution}

The least squares solution is the minima of $\|\mathbf{J} \mathbf{c}-\mathbf{z}\|^{2}$,

$$
\begin{aligned}
\|\mathbf{J} \mathbf{c}-\mathbf{z}\|^{2} & =(\mathbf{J} \mathbf{c}-\mathbf{z})^{\top}(\mathbf{J} \mathbf{c}-\mathbf{z}) \\
= & \mathbf{c}^{\top} \mathbf{J}^{\top} \mathbf{J} \mathbf{c} \underbrace{-\mathbf{c}^{\top} \mathbf{J}^{\top} \mathbf{z}-\mathbf{z}^{\top} \mathbf{J} \mathbf{c}}_{-2 \mathbf{c}^{\top} \mathbf{J}^{\top} \mathbf{z}}+\mathbf{z}^{\top} \mathbf{z} \\
\arg \min _{\mathbf{c}}\|\mathbf{J} \mathbf{c}-\mathbf{z}\|^{2} & \rightarrow \frac{\partial\|\mathbf{J} \mathbf{c}-\mathbf{z}\|^{2}}{\partial \mathbf{c}}=0 \\
\frac{\partial\|\mathbf{J} \mathbf{c}-\mathbf{z}\|^{2}}{\partial \mathbf{c}} & =2 \mathbf{J}^{\top} \mathbf{J} \mathbf{c}-2 \mathbf{J}^{\top} \mathbf{z} \\
\not 2 \mathbf{J}^{\top} \mathbf{J} \mathbf{c} & =\not 2 \mathbf{J}^{\top} \mathbf{z} \\
\mathbf{c} & =\left(\mathbf{J}^{\top} \mathbf{J}\right)^{-1} \mathbf{J}^{\top} \mathbf{z}
\end{aligned}
$$

which is the Moore-Penrose pseudoinverse of the problem $\mathbf{J c}=\mathbf{z} \rightarrow \mathbf{c}=\mathbf{J}^{\dagger} \mathbf{z}$ for $\mathbf{A}$ with linearly independent columns.

\section{B.2 Adding Penalty Terms}

To extend the least squares solution towards a generalized Tikhonov regularization framework is a matter of substitution:

$$
\|\mathbf{J} \mathbf{c}-\mathbf{z}\|^{2} \rightarrow\|\mathbf{J} \mathbf{c}-\mathbf{z}\|^{2}+\left\|\lambda \mathbf{L}\left(\mathbf{c}-\mathbf{c}_{0}\right)\right\|^{2}
$$




$$
\begin{array}{r}
{\left[\begin{array}{c}
\mathbf{J} \\
\lambda \mathbf{L}
\end{array}\right] \mathbb{C}=\left[\begin{array}{c}
\mathbf{z} \\
\lambda \mathbf{L} \mathbf{c}_{0}
\end{array}\right]} \\
\mathbf{J} \rightarrow\left[\begin{array}{c}
\mathbf{J} \\
\lambda \mathbf{L}
\end{array}\right], \quad \mathbf{z} \rightarrow\left[\begin{array}{c}
\mathbf{z} \\
\lambda \mathbf{L} \mathbf{c}_{0}
\end{array}\right]
\end{array}
$$

Substituting into (B.7), gives the following:

$$
\mathbf{c}=\left(\left[\begin{array}{ll}
\mathbf{J}^{\top} & \lambda \mathbf{L}^{\top}
\end{array}\right]^{\top}\left[\begin{array}{c}
\mathbf{J} \\
\lambda \mathbf{L}
\end{array}\right]\right)^{-1}\left[\begin{array}{ll}
\mathbf{J}^{\top} & \lambda \mathbf{L}^{\top}
\end{array}\right]^{\top}\left[\begin{array}{c}
\mathbf{z} \\
\lambda \mathbf{L} \mathbf{c}_{0}
\end{array}\right]
$$

Multiplied out, this gives

$$
\mathbf{c}=\left(\mathbf{J}^{\top} \mathbf{J}+\lambda^{2} \mathbf{L}^{\top} \mathbf{L}\right)^{-1}\left(\mathbf{J}^{\top} \mathbf{z}+\lambda^{2} \mathbf{L}^{\top} \mathbf{L} \mathbf{c}_{0}\right)
$$

\section{B.3 Generalized Tikhonov Solution}

The generalized Tikhonov solution extends the least squares solution to one using an arbitrary norm $\mathbf{W}$ on the solution error term. This allows less reliable data to be weighted differently.

$$
\begin{aligned}
\|\mathbf{J} \mathbf{c}-\mathbf{z}\|_{\mathbf{W}}^{2} & =(\mathbf{J} \mathbf{c}-\mathbf{z})^{\top} \mathbf{W}(\mathbf{J} \mathbf{c}-\mathbf{z}) \\
& =\mathbf{c}^{\top} \mathbf{J}^{\top} \mathbf{W} \mathbf{J} \mathbf{c} \underbrace{-\mathbf{c}^{\top} \mathbf{J}^{\top} \mathbf{W} \mathbf{z}-\mathbf{z}^{\top} \mathbf{W}^{\top} \mathbf{J} \mathbf{c}}_{-2 \mathbf{c}^{\top} \mathbf{J}^{\top} \mathbf{W} \mathbf{z}}+\mathbf{z}^{\top} \mathbf{W} \mathbf{z} \\
\frac{\partial\|\mathbf{J} \mathbf{c}-\mathbf{z}\|^{2}}{\partial \mathbf{c}} & =2 \mathbf{J}^{\top} \mathbf{W} \mathbf{J} \mathbf{c}-2 \mathbf{J}^{\top} \mathbf{W} \mathbf{z}
\end{aligned}
$$




$$
\mathbf{c}=\left(\mathbf{J}^{\top} \mathbf{W} \mathbf{J}\right)^{-1} \mathbf{J}^{\top} \mathbf{W} \mathbf{z}
$$

Performing the same substitutions as in Section B.2, but with the additional norm $\mathbf{W}$, gives

$$
\mathbf{J} \rightarrow\left[\begin{array}{c}
\mathbf{J} \\
\lambda \mathbf{L}
\end{array}\right], \quad \mathbf{z} \rightarrow\left[\begin{array}{c}
\mathbf{z} \\
\lambda \mathbf{L} \mathbf{c}_{0}
\end{array}\right], \quad \mathbf{W} \rightarrow\left[\begin{array}{cc}
\mathbf{W} & 0 \\
0 & \mathbf{I}
\end{array}\right]
$$

and substituting it into (B.7), gives

$$
\mathbf{c}=\left(\left[\begin{array}{ll}
\mathbf{J}^{\top} & \lambda \mathbf{L}^{\top}
\end{array}\right]^{\top}\left[\begin{array}{cc}
\mathbf{W} & 0 \\
0 & \mathbf{I}
\end{array}\right]\left[\begin{array}{c}
\mathbf{J} \\
\lambda \mathbf{L}
\end{array}\right]\right)^{-1}\left[\begin{array}{ll}
\mathbf{J}^{\top} & \lambda \mathbf{L}^{\top}
\end{array}\right]^{\top}\left[\begin{array}{cc}
\mathbf{W} & 0 \\
0 & \mathbf{I}
\end{array}\right]\left[\begin{array}{c}
\mathbf{z} \\
\lambda \mathbf{L} \mathbf{c}_{0}
\end{array}\right]
$$

Multiplied out, this gives

$$
\mathbf{c}=\left(\mathbf{J}^{\top} \mathbf{W} \mathbf{J}+\lambda^{2} \mathbf{L}^{\top} \mathbf{L}\right)^{-1}\left(\mathbf{J}^{\top} \mathbf{W} \mathbf{z}+\lambda^{2} \mathbf{L}^{\top} \mathbf{L} \mathbf{c}_{\mathbf{0}}\right)
$$

This solution with a null prior $\left(\mathbf{c}_{0}=0\right)$ is what is implemented as a single step solver in the Electrical Impedance and Diffuse Optics Reconstruction Software (EIDORS) package.[10]

\section{B.4 Wiener Filter Form}

A Wiener filter form [69] is useful because it allows isolation of the Signal-to-Noise Ratio (SNR) where a Wiener filter takes the following form

$$
\mathbf{c}=\mathbf{J}^{*}\left(\mathbf{J J}^{*}+\mathrm{SNR}\right)^{-1} \mathbf{z}
$$


The transformation of the generalized Tikhonov solution (B.19) to a Wiener filter form requires rearranging the solution such that

$$
\begin{aligned}
& \mathbf{c}=\left(\mathbf{J}^{\top} \mathbf{W} \mathbf{J}+\lambda^{2} \mathbf{L}^{\top} \mathbf{L}\right)^{-1}\left(\mathbf{J}^{\top} \mathbf{W}\left(\mathbf{z}-\mathbf{J} \mathbf{c}_{0}+\mathbf{J} \mathbf{c}_{0}\right)+\lambda^{2} \mathbf{L}^{\top} \mathbf{L} \mathbf{c}_{0}\right) \\
& \mathbf{c}=\left(\mathbf{J}^{\top} \mathbf{W} \mathbf{J}+\lambda^{2} \mathbf{L}^{\top} \mathbf{L}\right)^{-1}\left(\mathbf{J}^{\top} \mathbf{W}\left(\mathbf{z}-\mathbf{J} \mathbf{c}_{0}\right)+J^{\top} \mathbf{W} \mathbf{J} \mathbf{c}_{0}+\lambda^{2} \mathbf{L}^{\top} \mathbf{L} \mathbf{c}_{0}\right) \\
& \mathbf{c}=\left(\mathbf{J}^{\top} \mathbf{W} \mathbf{J}+\lambda^{2} \mathbf{L}^{\top} \mathbf{L}\right)^{-1}\left(\mathbf{J}^{\top} \mathbf{W}\left(\mathbf{z}-\mathbf{J} \mathbf{c}_{0}\right)\right) \\
& \quad+\underbrace{\left(\mathbf{J}^{\top} \mathbf{W} \mathbf{J}+\lambda^{2} \mathbf{L}^{\top} \mathbf{L}\right)^{-1}\left(J^{\top} \mathbf{W} \mathbf{J}+\lambda^{2} \mathbf{L}^{\top} \mathbf{L}\right)}_{\mathbf{I}} \mathbf{c}_{0} \\
& \mathbf{c}=\left(\mathbf{J}^{\top} \mathbf{W} \mathbf{J}+\lambda^{2} \mathbf{L}^{\top} \mathbf{L}\right)^{-1}\left(\mathbf{J}^{\top} \mathbf{W}\left(\mathbf{z}-\mathbf{J} \mathbf{c}_{0}\right)\right)+\mathbf{c}_{0} \\
& \mathbf{c}-\mathbf{c}_{0}=\left(\mathbf{J}^{\top} \mathbf{W} \mathbf{J}+\lambda^{2} \mathbf{L}^{\top} \mathbf{L}\right)^{-1} \mathbf{J}^{\top} \mathbf{W}\left(\mathbf{z}-\mathbf{J} \mathbf{c}_{0}\right)
\end{aligned}
$$

followed by the application of the so-called "PosDef" matrix identity [68, (173)]

$$
\left(\mathbf{P}^{-1}+\mathbf{B}^{\top} \mathbf{R}^{-1} \mathbf{B}\right)^{-1} \mathbf{B}^{\top} \mathbf{R}^{-1}=\mathbf{P B}^{\top}\left(\mathbf{B P B}^{\top}+\mathbf{R}\right)^{-1}
$$

where $\mathbf{P}$ and $\mathbf{R}$ are positive definite matrices that are invertable and

$$
\mathbf{B}=\mathbf{J}, \quad \mathbf{P}=\left(\mathbf{L}^{\top} \mathbf{L}\right)^{-1}, \quad \mathbf{R}=\left(\lambda^{-2} \mathbf{W}\right)^{-1}
$$

Applying this identity to the rearranged generalized Tikhonov solution (B.25) gives

$$
\begin{aligned}
& \mathbf{c}-\mathbf{c}_{0}=\left(\mathbf{L}^{\top} \mathbf{L}\right)^{-1} \mathbf{J}^{\top}\left(\mathbf{J}\left(\mathbf{L}^{\top} \mathbf{L}\right)^{-1} \mathbf{J}^{\top}+\lambda^{2} \mathbf{W}^{-1}\right)^{-1}\left(\mathbf{z}-\mathbf{J} \mathbf{c}_{0}\right) \\
& \mathbf{c}=\left(\mathbf{L}^{\top} \mathbf{L}\right)^{-1} \mathbf{J}^{\top}\left(\mathbf{J}\left(\mathbf{L}^{\top} \mathbf{L}\right)^{-1} \mathbf{J}^{\top}+\lambda^{2} \mathbf{W}^{-1}\right)^{-1}\left(\mathbf{z}-\mathbf{J} \mathbf{c}_{0}\right)+\mathbf{c}_{0}
\end{aligned}
$$




\section{References}

[1] W. R. Breckon, "Image reconstruction in electrical impedance tomography," Ph.D. dissertation, 1990.

[2] M. Cheney, D. Isaacson, and J. C. Newell, "Electrical impedance tomography," SIAM review, vol. 41, no. 1, pp. 85-101, 1999.

[3] D. Holder, Ed., Electrical impedance tomography: Methods, history and applications. IOP Publishing Ltd, 2005.

[4] S. E. Mirvis and K. Shanmuganathan, Imaging in trauma and critical care, 2nd ed. Saunders, 2003.

[5] S. Webb, The physics of medical imaging. Taylor \& Francis, 1988.

[6] E. P. Steinberg, "The status of MRI in 1986: rates of adoption in the United States and worldwide," American Journal of Roentgenology, vol. 147, no. 3, p. $453,1986$.

[7] T. J. C. Faes, H. R. van Genderingen, and A. V. Noordegraaf, "Some reflections on the EIT Conference (London, UK, 22-24 June 2005)," Physiol. Meas., vol. 27, 2006. 
[8] A. Boyle, W. R. B. Lionheart, and A. Adler, "Evaluating deformation corrections in electrical impedance tomography," in 9th Conf. Electrical Impedance Tomography, Dartmouth College, Hannover, NH, USA, Jun. 2008.

[9] …, "Artifacts due to conformal deformations in electrical impedance tomography," in 10th Conf. Electrical Impedance Tomography, University of Manchester, Manchester, UK, Jun. 2009.

[10] A. Adler and W. R. B. Lionheart, "Uses and abuses of EIDORS: An extensible software base for EIT," Physiol. Meas., vol. 27, no. 5, pp. S25-S42, May 2006.

[11] (2010, Jan.) tomography. Merriam-Webster Online. [Online]. Available: http://www.merriam-webster.com/dictionary/tomography

[12] F. Natterer, The Mathematics of Computerized Tomography. SIAM, 2001.

[13] C. Dalziel and F. Massoglia, "Let-go currents and voltages," AIEE Trans, vol. 75, no. part II, pp. 49-56, 1956.

[14] J. G. Webster, Ed., Medical Instrumentation: Application and Design, 3rd ed. New York: John Wiley \& Sons, 1998.

[15] C. F. Dalziel, "Electric shock," Advances in biomedical engineering, vol. 3, pp. 223-248, 1973.

[16] IEC60601-1: Medical electrical equipment - Part 1: General requirements for basic safety and essential performance, 3rd ed. International Electrotechnical Commission, 2005. 
[17] R. P. Henderson and J. G. Webster, "An impedance camera for spatially specific measurements of the thorax," IEEE Trans. Biomed. Eng., pp. 250-254, 1978.

[18] A. L. Benabid, L. Balme, J. C. Persat, M. Belleville, J. P. Chirossel, M. BuyleBodin, J. de Rougemont, and C. Poupot, "Electrical impedance brain scanner: principles and preliminary results of simulation," T.-I.-T. journal of life sciences, vol. 8, no. 1-2, p. 59, 1978.

[19] B. H. Brown and A. D. Seagar, "The sheffield data collection system," Clinical Physics and Physiological Measurement, vol. 8, no. 4A, pp. 91-97, 1987.

[20] D. C. Barber, B. H. Brown, and I. L. Freeston, "Experimental results of electrical impedance imaging," in Proc VIth Int. ConJ Electrical Bioimpedance (ICEBI), Zadar, Yugoslauia, 1983, pp. 1-5.

[21] D. C. Barber and B. H. Brown, "Applied potential tomography," Journal of Physics E: Scientific Instruments, vol. 17, pp. 723-733, 1984.

[22] I. Frerichs, "Electrical impedance tomography (EIT) in applications related to lung and ventilation: a review of experimental and clinical activities," Physiol. Meas., vol. 21, no. 2, pp. 1-21, 2000.

[23] K. Boone, D. C. Barber, and B. H. Brown, "Imaging with electricity: report of the European concerted action on impedance tomography," Journal of medical engineering \&s technology, vol. 21, no. 6, pp. 201-232, 1997.

[24] O. C. Zienkiewicz, R. L. Taylor, and J. Z. Zhu, The Finite Element Method: Its Basis and Fundamentals. Butterworth-Heinemann, 2005. 
[25] C. Gòmez-Laberge, "Electrical Impedance Tomography for Deformable Media," Master's thesis, 2006.

[26] B. Graham, "Enhancements in EIT Image Reconstruction for 3D Lung Imaging," Ph.D. dissertation, 2007.

[27] P. C. Hansen, Rank-deficient and discrete ill-posed problems: numerical aspects of linear inversion. Society for Industrial Mathematics, 1998.

[28] J. P. Kaipio, V. Kolehmainen, E. Somersalo, and M. Vauhkonen, "Statistical inversion and Monte Carlo sampling methods in EIT," Inv. Prob., vol. 16, pp. 1487-1522, 2000.

[29] J. Hadamard, "Sur les problemes aux derivees partielles et leur signification physique," Princeton University Bulletin, vol. 13, no. 1, pp. 49-52, 1902.

[30] A. P. Calderón, "On an inverse boundary value problem," Computational \& Applied Mathematics, vol. 25, pp. 133-138, 2006, (reprint, orig. 1980).

[31] A. I. Nachman, "Global uniqueness for a two-dimensional inverse boundary value problem," The Annals of Mathematics, vol. 143, no. 1, pp. 71-96, 1996.

[32] K. S. Cheng, D. Isaacson, J. C. Newell, and D. G. Gisser, "Electrode models for electric current computed tomography," IEEE Trans. Biomed. Eng., vol. 36, no. 9, pp. 918-924, 1989.

[33] E. Somersalo, M. Cheney, and D. Isaacson, "Existence and uniqueness for electrode models for electric current computed tomography," SIAM J. Appl. Math., vol. 52, no. 4, pp. 1023-1040, 1992. 
[34] T. J. Yorkey, J. G. Webster, and W. J. Tompkins, "Comparing reconstruction algorithms for electrical impedance tomography," IEEE Trans. Biomed. Eng., pp. 843-852, 1987.

[35] M. Vauhkonen, D. Vadasz, P. A. Karjalainen, E. Somersalo, and J. P. Kaipio, "Tikhonov regularization and prior information in electrical impedance tomography," IEEE transactions on medical imaging, vol. 17, no. 2, pp. 285-293, 1998.

[36] M. Vauhkonen, W. R. B. Lionheart, L. M. Heikkinen, P. J. Vauhkonen, and J. P. Kaipio, "A MATLAB package for the EIDORS project," Physiol. Meas., vol. 22, pp. 107-111, 2001.

[37] A. N. Tikhonov and V. Y. Arsenin, Solutions of ill-posed problems. V. H. Winston and Sons (distributed by Wiley, New York), 1977, translation by F. John.

[38] C. R. Vogel, Computational methods for inverse problems. Society for Industrial Mathematics, 2002.

[39] J. P. Kaipio and E. Somersalo, Statistical and computational inverse problems. Springer, 2005.

[40] M. Soleimani, C. E. Powell, and N. Polydorides, "Improving the forward solver for the complete electrode model in EIT using algebraic multigrid," IEEE Trans. Med. Imag., vol. 24, no. 5, pp. 577-583, 2005.

[41] V. A. Morozov, "On the solution of functional equations by the method of regularization," in Soviet Math. Dokl, vol. 7, 1966, pp. 414-417. 
[42] P. C. Hansen, "Analysis of discrete ill-posed problems by means of the L-curve," SIAM review, vol. 34, no. 4, pp. 561-580, 1992.

[43] B. M. Graham and A. Adler, "Objective selection of hyperparameter for EIT," Physiol. Meas., vol. 27, pp. S65-S79, 2006.

[44] D. C. Barber and B. H. Brown, "Errors in reconstruction of resistivity images using a linear reconstruction technique," Clin. Phys. Physiol. Meas., vol. 9, pp. 101-104, 1988.

[45] N. Harris, A. Suggett, D. Barber, and B. Brown, "Applied potential tomography: a new technique for monitoring pulmonary function," Clin. Phys. Physiol. Meas., vol. 9, pp. 79-85, 1988.

[46] A. Adler, R. Guardo, and Y. Berthiaume, "Impedance imaging of lung ventilation: do we need to account for chest expansion?" IEEE Trans. Biomed. Eng., vol. 43, no. 4, pp. 414-420, Apr. 1996.

[47] J. Zhang and R. P. Patterson, "EIT images of ventilation: what contributes to the resistivity changes?" Physiol. Meas., vol. 26, p. 81, 2005.

[48] W. R. B. Lionheart, "Boundary shape and electrical impedance tomography," Inv. Prob., vol. 14, no. 1, pp. 139-147, Feb. 1998.

[49] B. H. Blott, G. J. Daniell, and S. Meeson, "Electrical impedance tomography with compensation for electrode positioning variations," Phys. Med. Biol., vol. 43, pp. 1731-1739, 1998. 
[50] V. Kolehmainen, M. Lassas, and P. Ola, "The inverse conductivity problem with an imperfectly known boundary," SIAM J. Appl. Math., vol. 66, no. 2, pp. 365-383, 2006.

[51] M. Soleimani, C. Gómez-Laberge, and A. Adler, "Imaging of conductivity changes and electrode movement in EIT," Physiol. Meas., vol. 27, no. 5, pp. S103-S113, May 2006.

[52] S. Seydnejad and H. Fahimi, "A new method for finding the exact electrode location and bodyperimeter used for EIT," in Engineering in Medicine and Biology Society, 1994. Engineering Advances: New Opportunities for Biomedical Engineers. Proceedings of the 16th Annual International Conference of the IEEE, 1994, pp. 541-542.

[53] J. M. Khor, A. Tizzard, A. Demosthenous, and R. Bayford, "Development of a Sensor Network for Dynamic Boundary Measurement in Neonatal Electrical Impedance Tomography (EIT)," in World Congress on Medical Physics and Biomedical Engineering September 7-12, 2009 Munich, Germany: Vol. 25/2 Diagnostic Imaging. Springer, 2009, p. 386.

[54] C. Gómez-Laberge and A. Adler, "Direct EIT Jacobian calculations for conductivity change and electrode movement," Physiol. Meas., vol. 29, pp. S89-S99, 2008.

[55] N. Joukowski, "On the profiles of airfoils," Z. Flugtech. Motorluftschiffahrt., vol. 1, p. 281, 1910, vol. 3, p. 81, 1912 . 
[56] N. Coulombe, H. Gagnon, F. Marquis, Y. Skrobik, and R. Guardo, "A parametric model of the relationship between EIT and total lung volume," Physiol. Meas., vol. 26, no. 4, pp. 401-411, Aug. 2005.

[57] S. Goldberg, Curvature and Homology. Dover Publications, 1982.

[58] M. Schottenloher, A Mathematical Introduction to Conformal Field Theory. Springer, 2008, ch. Sec 2.4 .

[59] J. Marsden and M. Hoffman, Basic Complex Analysis. W.H. Freeman \& Company, 1998.

[60] T. Vilhunen, J. P. Kaipio, P. J. Vauhkonen, T. Savolainen, and M. Vauhkonen, "Simultaneous reconstruction of electrode contact impedances and internal electrical properties: I. Theory," Meas. Sci. and Tech., vol. 13, no. 12, pp. $1848-1854,2002$.

[61] L. M. Heikkinen, T. Vilhunen, R. M. West, and M. Vauhkonen, "Simultaneous reconstruction of electrode contact impedances and internal electrical properties: II. Laboratory experiments," Meas. Sci. and Tech., vol. 13, no. 12, pp. 1855$1861,2002$.

[62] P. Hua, E. J. Woo, J. G. Webster, and W. J. Tompkins, "Finite element modeling of electrode-skin contact impedance inelectrical impedance tomography," IEEE Trans. Biomed. Eng., vol. 40, no. 4, pp. 335-343, 1993.

[63] E. T. McAdams, J. Jossinet, A. Lackermeier, and F. Risacher, "Factors affecting electrode-gel-skin interface impedance in electrical impedance tomography," 
Medical and Biological Engineering and Computing, vol. 34, no. 6, pp. 397-408, 1996.

[64] N. Polydorides and W. R. B. Lionheart, "A Matlab toolkit for three-dimensional electrical impedance tomography: a contribution to the Electrical Impedance and Diffuse Optical Reconstruction Software project," Meas. Sci. and Tech., vol. 13, no. 12, pp. 1871-1883, 2002.

[65] V. Kolehmainen, M. Vauhkonen, P. A. Karjalainen, and J. P. Kaipio, "Assessment of errors in static electrical impedance tomography with adjacent and trigonometric current patterns," Physiol. Meas., vol. 18, pp. 289-303, 1997.

[66] K. G. Boone and D. S. Holder, "Effect of skin impedance on image quality and variability in electrical impedance tomography: a model study," Medical and Biological Engineering and Computing, vol. 34, no. 5, pp. 351-354, 1996.

[67] M. Lovric, Ed., Vector Calculus. Wiley, 2007.

[68] K. B. Petersen and M. S. Pedersen, The Matrix Cookbook, 2008.

[69] N. Wiener, Extrapolation, Interpolation, and Smoothing of Stationary Time Series. Wiley, 1949. 


\section{Vitae}

Name:

Research Interests: Electrical Impedance Tomography, the Finite Element Method, and Inverse Problems

Education:

Experience:

Awards:

Alistair James Scott Boyle, PEng.

MASc Student, Biomedical Engineering, Carleton University, 2007-2010

BSc, Electrical Engineering, University of Calgary, 19972002

Design Engineer, Freescale Semiconductors, 2005-Present Design \& Verification Engineer, Founder, Seaway Networks, 2001-2005

Verification Engineer, Nortel, 2000-2001

Entry Scholarship (Masters), 2007

EnCana Scholarship, 1997-2001 